Portland State University

PDXScholar

$12-12-1979$

\title{
Effect of Phenoxy Acids and Their Derivatives on the Ionic Permeability of Bilayer Lipid Membranes
}

Malkanthi Paulis Illangasekare

Portland State University

Follow this and additional works at: https://pdxscholar.library.pdx.edu/open_access_etds

Part of the Environmental Sciences Commons

Let us know how access to this document benefits you.

\section{Recommended Citation}

Illangasekare, Malkanthi Paulis, "Effect of Phenoxy Acids and Their Derivatives on the Ionic Permeability of Bilayer Lipid Membranes" (1979). Dissertations and Theses. Paper 850.

https://doi.org/10.15760/etd.850

This Dissertation is brought to you for free and open access. It has been accepted for inclusion in Dissertations and Theses by an authorized administrator of PDXScholar. Please contact us if we can make this document more accessible: pdxscholar@pdx.edu. 
EFFECT OF PHENOXY ACIDS AND THEIR DERIVATIVES ON THE IONIC PERMEABILITY OF BILAYER LIPID MEMBRANES

by

MALKANTHI PAULIS ILLANGASEKARE

A dissertation submitted in partial fulfillment of the requirements for the degree of

DOCTOR OF PHILOSOPHY in

ENVIRONMENTAL SCIENCES AND RESOURCES -- PHYSICS

Portland State University

1979

(1980 Malkanthi Paulus IIlangasekare 
TO THE OFFICE OF GRADUATE STUDIES AND RESEARCH:

The members of the Committee approve the dissertation of

Malkanthi Paulis Illangasekare presented December 12, 1979.
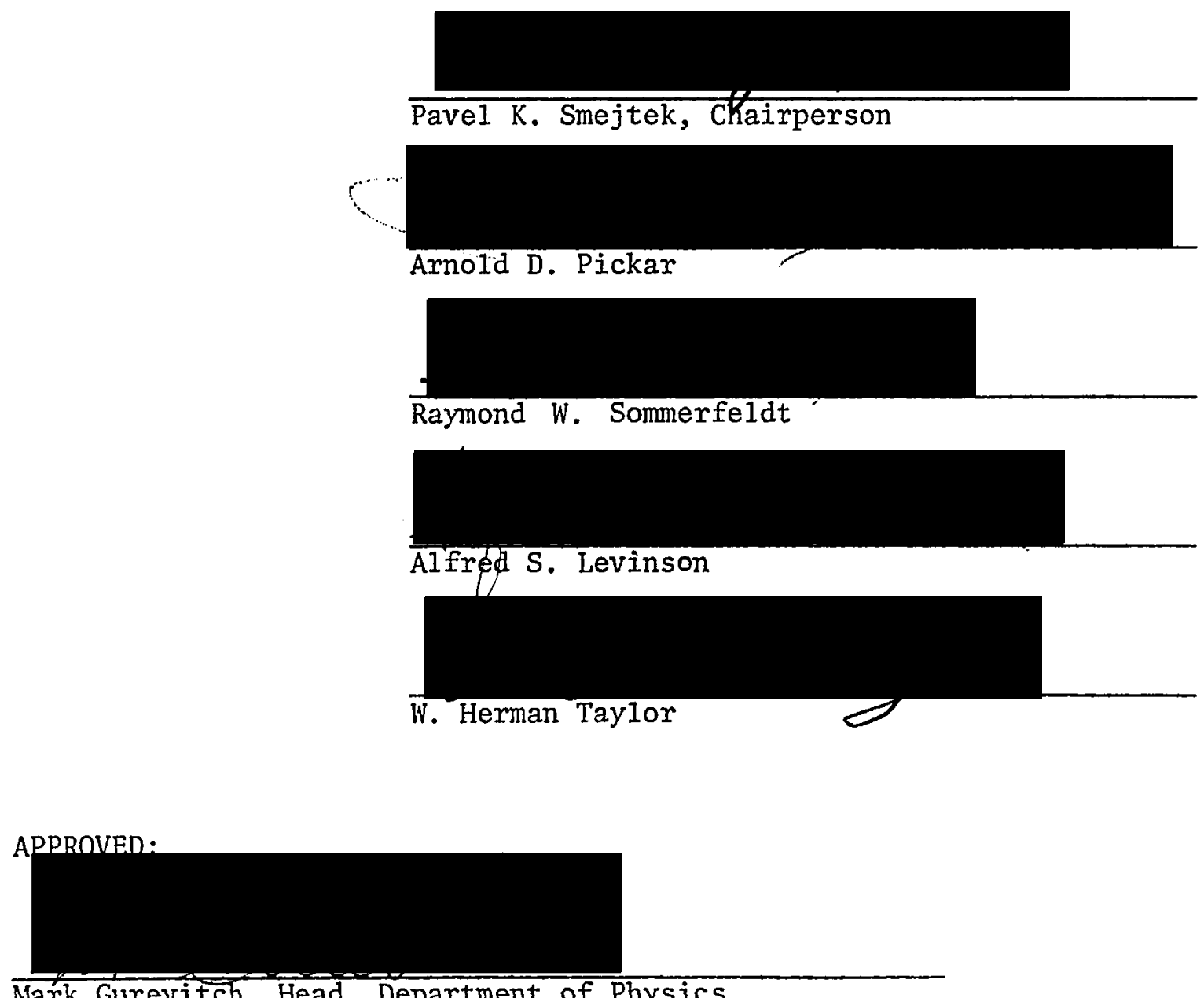

Mark Gurevitch, Head, Department of Physics

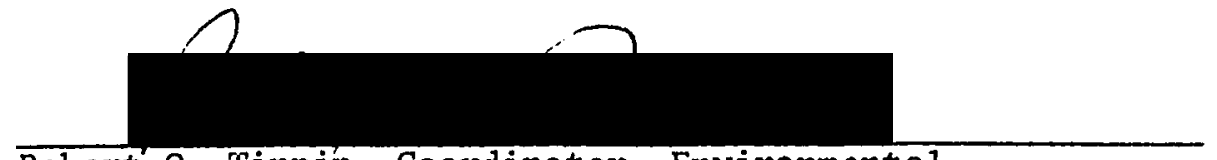

Robert 0. Tinnin, Coordinator, Environmental

Sciences and Resources Ph.D. Program

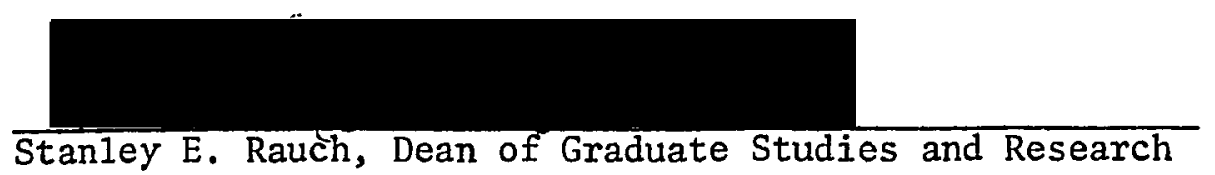


AN ABSTRACT OF THE DISSERTATION OF Malkanthi Paulis Illangasekare for the Doctor of Philosophy in Environmental Sciences and Resources-Physics presented December 12, 1979.

Title: Effect of Phenoxy Acids and Their Derivatives on the Ionic Permeability of Bilayer Lipid Membranes.

APPROVED BY MEMBERS OF THE DISSERTATION COMMITTEE:

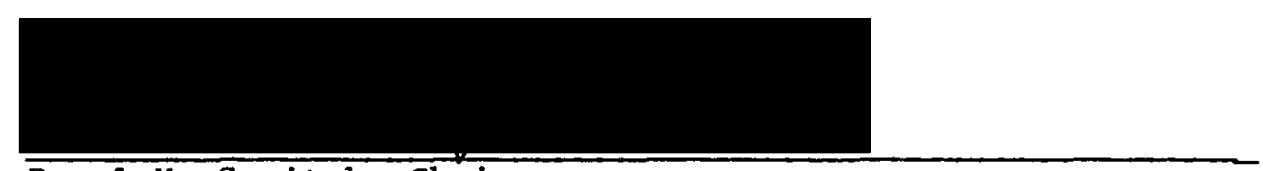

Pavel K. Smejtek, Chairperson

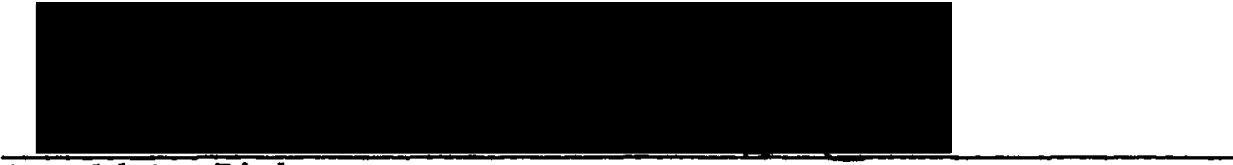
Arnold D. Pickar

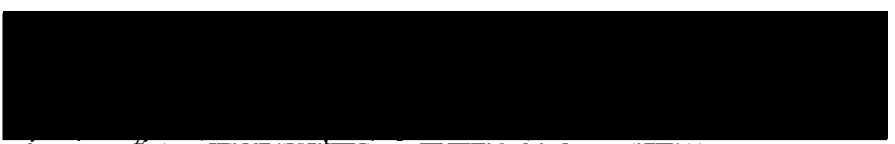

Raymond W. Sommerfeldt

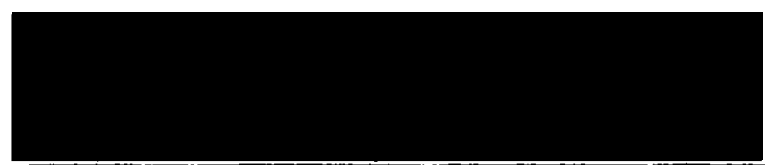

A1fred S. Levinson

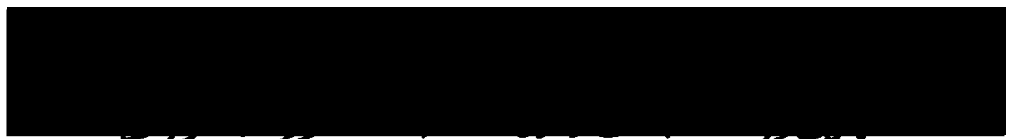

W. Herman Taylor 


\section{ABSTRACT}

It has been found that the herbicide 2,4-D has the ability to increase the rate of transport of positive ions of several kinds and inhibit the transport of negatively charged tetraphenylborate ions in lipid bilayer membranes. Only the neutral molecules of 2,4-D are transport active. The ionized 2,4-D molecules do not modify the transport of ions, and do not by themselves permeate through lipid membranes. The results suggest that the enhancement of transport of positively charged ions is dominated by the increase of the ion translocation rate constant. It has been shown that membrane transport of negatively charged tetraphenylborate ions is suppressed by 2,4-D. The effect is dominated by the suppression of translocation of these ions across membrane interior, rather than by the decrease of their adsorption at the membrane surface. It has been shown that the enhancement of nonactin-mediated transport of potassium ions by 2,4-D can be accounted for by a simple carrier model. From the changes of kinetic parameters of nonactin- $\mathrm{K}^{+}$transport, membrane conductance due to positively charged tetraphenylarsonium ions and also from the changes of membrane conductance and relaxation time constant due to transport of negatively charged tetraphenylborate ions, the changes of the electric potential of the membrane interior have been estimated. The potential of the membrane interior becomes more negative in the presence of 2,4-D and its change is proportional to the aqueous concentration of 2,4-D. The effect of 2,4-D on ion transport was explained by the hypothesis that a layer of 2,4-D molecules is absorbed within the membrane/water interfacial region, and that the 2,4-D molecules are 
oriented in such a way that their dipole moment is directed toward the aqueous medium. The results suggest that this layer is located in the hydrocarbon side of the interface. The hypothesis has been confirmed by the measurements of changes of electric potential difference across air/water and air/lipid monolayer/water interfaces. It has been found that the electric potential of the nonpolar side of the interface decreases in the presence of neutral molecules of 2,4-D, which is in agreement with the conclusions drawn from the results of membrane experiments.

The effect of the other auxin-type phenoxy herbicides, 2,4,5-T and 2,4-DB on lipid bilayer membranes has been found to be similar to that of 2,4-D. In contrast, the phenoxy acid $2,4,6-T$, has very little or no herbicidal activity, and at the same time has small effect on ion transport in membranes.

Biologically active 2,4-D derivatives, amino acid conjugates of 2,4-D (isoleucine, leucine and valine conjugates) have been found to be also transport active in a manner similar to 2,4-D. Similar conclusions have been drawn from experiments with natural auxin indole acetic acid.

The results obtained in this work suggest the existence of correlation between the biological activity of herbicides acting as plant growth regulators and their ability to enhance transport of positively charged ions across lipid membranes. This work provides insight into the physical origin of such activity. 


\section{ACKNOWLEDGMENTS}

I wish to express a great deal of appreciation and my sincere gratitude to Dr. Pavel Smejtek for his invaluable guidance and encouragement. Also, I wish to thank Dr. Kwan Hsu for introducing me to many experimental methods and Dr. Amold Pickar for his suggestions and recommendations in the preparation of this manuscript.

I would also like to thank $\mathrm{Dr}$. Alfred Levinson, who synthesized the amino acid conjugates of 2,4-D, that have been used in the present work.

My gratitude goes to the members of the supervisory committee for their helpful comments.

Finally, I would like to thank my husband, Dr. Tissa Illangasekare, for the sacrifices he made during my course of studies and to my parents, who have encouraged me to pursue higher education. This dissertation is dedicated to my parents.

This investigation was supported by Grant 2R01 ES 00937-04 from the National Institute of Health. 
TABLE OF CONTENTS

PAGE

ACKNOWLEDGMENTS ................. . iii

LIST OF TABLES. ................ vii

LIST OF FIGURES . . . . . . . . . . . . . viii

CHAPTER

I INTRODUCTION. . . . . . . . . . . . . I

1a. Biomembranes and artificial lipid bilayer

membranes .. . . . . . . . . . . 1

1b. Biological activity of phenoxy pesticides

and other related substances. . . . . . . 3

II GENERAL CONSIDERATIONS REGARDING THE MECHANISM

OF LIPID BILAYER MEMBRANES IN THE PRESENCE

OF PHENOXY PESTICIDES AND THEIR DERIVATIVES. . . • 5

2a. Diffusion of anions. . . . . . . . . . 5

2b. "Uncoupler type" of membrane electrical

conductivity induced by phenoxy acids . . . 6

2c. Mediated cation transport. . . . . . . . 7

2d. Modification of membrane selectivity and

changes of kinetics of carrier mediated

ion transport in the presence of

phenoxy pesticides........... . 7

III THEORETICAL CONCEPTS OF ION TRANSPORT

IN LIPID BILAYER . . . . . . . . . . . . . . . . 10

3a. Energy barriers and ion transport. . . . . . 10

3b. The carrier model for nonactin-

mediated $\mathrm{K}^{+}$transport .......... 18

3c. Transport of $\mathrm{TPhAs}^{+}$ions across

the membrane. . . . . . . . . . . . 21 
3d. Transport of $\mathrm{TPhB}^{-}$ions across

lipid membranes. . . . . . . . . . 22

4a. Membrane formation and methods of preparation of solutions . . . . . . . 28

4b. Steady state conduction measurements. . . . 29

4c. Transient current measurements and analysis of data......... 30

4d. Interfacial potential measurements. . . . . 32

4e. Materials.............. 33

V EXPERIMENTAL RESULTS ............. 55

5a. Studies of the effect of 2,4-D on membrane transport . . . . . . . . 35

5b. Studies of the 2,4-D enhanced transport of positive ions . . . . . . . . . . . . 39

5c. Studies of 2,4-D suppressed transport of negatively charged tetraphenylborate: Effect of $\mathrm{TPhB}^{-}$induced membrane conductance, relaxation time constant and net transfer of charge . . . . . . . 43

5d. Ionic strength dependence of membrane conductance. . . . . . . . . . . 48

5e. Relationship between the membrane structure and the effect of 2,4-D induced modification of membrane ion transport. . . . . . . . . . . 56

5f. Electrical potential difference across air/ water and air/lipid monolayer/water interfaces . . . . . . . . . . . .

5g. Experiments with amino acid conjugates of 2,4-D................

5h. Experiments with natural growth hormone indole acetic acid . . . . . . . . 72

5i. Experiments with 2,4-DB, 2,4,5-T, 2,4,6-T and $2,4-\mathrm{D}$-isobutyl ester ........ 
6a. Membrane conductance induced by phenoxy acids amino acid conjugates of 2,4-D and indole acetic acid. . . . . .

6b. Effect of 2,4-D on kinetics of carrier-mediated potassium ion transport across membrane. . . . . . . 80

6c. Effect of 2,4-D on $\mathrm{TPhB}^{-}$conductance...... 90

6d. 2,4-D induced changes of electric potential of membrane interior . . . . . 96

6e. 2,4-D induced changes of interfacial electric potential difference. . . . . . 109

6f. Biological significance . . . . . . . . 112

6g. Modification of ion transport in lipid membranes by amino acid conjugates of 2,4-D, indole acetic acid, 2,4,5-T, $2,4,6-T, 2,4-D B$ and 2,4-D-isobutyl ester . . 114

VII CONCLUSIONS, HYPOTHESES AND PROPOSAL FOR FURTHER STUDIES . . . . . . . . . . . . 122

7a. Conclusions drawn from the investigation of the mechanism of membrane permeatoxicity of 2,4-D............ 122

7b. Conclusions and hypotheses drawn from the results of the studies of amino acid conjugates of $2,4-D$, indole acetic acid, $2,4-\mathrm{DB}, 2,4,5-\mathrm{T}, 2,4,6-\mathrm{T}$ and $2,4-\mathrm{D}$ ester................ . 125 


\section{LIST OF TABLES}

TABLE

PAGE

I

Change of kinetic parameters of nonactin- $\mathrm{K}^{+}$in

PC-chol membranes with 2,4-D concentration

as obtained from the potassium concentration

dependence. . . . . . . . . . . . 85

II Effects of 2,4-D concentration on kinetic

characteristics of nonactin $-\mathrm{K}^{+}$transport

in PC-chol membranes. ........... 86

III Correlation of biological and membrane

phenomena . . . . . . . . . . . . . 113 


\section{LIST OF FIGURES}

FIGURE

PAGE

The "image charge" barrier experienced by an ion crossing a bilayer membrane. . . . . . . 12

Mechanism of potassium ion transport across membrane by nonactin ........... 18

A block diagram of the experimental apparatus

used for the transient current measurements. . . 31

The structure of 2,4-D.............. 35

Effect of 2,4-D on the PC-chol membrane conductance due to nonactin, $\mathrm{TPhB}^{-}$and $\mathrm{TPhAs}^{+}$. . 37 $\mathrm{pH}$ dependence of $2,4-\mathrm{D}$ enhanced nonactin- $\mathrm{K}^{+}$ conductance of PC-chol membranes . . . . . 58

Control experiment. A demonstration that nonactin- $\mathrm{K}^{+}$conductance of PC-chol membranes is independent of $\mathrm{pH}$ in the absence of 2,4-D . . 40 2,4-D enhancement of nonactin- $\mathrm{K}^{+}$conductance of PC-chol membranes . . . . . . . . . . 4 41 Voltage dependence of $2,4-\mathrm{D}$ enhanced nonactin- $\mathrm{K}^{+}$ conductance. . . . . . . . . . . . 42

9a Dependence of 2,4-D enhanced nonactin- $K^{+}$conductance on potassium ion concentration. . . . 44

9b-d Effect of potassium ion concentration on voltage dependence of normalized membrane conductance at three concentrations of 2,4-D . . . . . . 45 
Voltage dependence of initial $\mathrm{TPhB}^{-}$

conductance of PC-chol membrane as a

function of aqueous concentration of 2,4-D. . . . 46

10b Voltage dependence of initial $\mathrm{TPhB}^{-}$

conductance of PC-chol membrane as a

function of aqueous concentration of 2,4-D. . . 47

11a Voltage dependence of membrane current

relaxation time constant as a function of

aqueous 2,4-D concentration ......... 49

$11 \mathrm{~b} \quad$ Voltage dependence of membrane current

relaxation time constant as a function of

aqueous 2,4-D concentration . . . . . . . 50

12 The effect of 2,4-D on the transfer of electric

charge associated with $\mathrm{TPhB}^{-}$ions across

PC-chol membranes............. 51

13a Effect of ionic strength on the magnitude of

membrane conductance associated with the

transport of nonactin $-\mathrm{K}^{+}$and $\mathrm{TPhAs}^{+}$ions

across the PC-chol membranes. . . . . . . . 53

13b Effect of ionic strength on the voltage dependence

of normalized membrane conductance associated

with transport of nonactin-K ${ }^{+}$complex . . . . . 54

13c Control experiment. A demonstration that in the absence of 2,4-D, the effect of ionic strength

on the voltage dependence of nonactin- $\mathrm{K}^{+}$

conductance is absent . . . . . . . . . . 55 
Changes of interfacial potential difference

across the PC-chol monolayer and the

air/aqueous solution interface as a

function of aqueous concentration

of 2,4-D................... 57

Changes of interfacial potential difference

across the PC-chol monolayer and the air/

aqueous solution interface as a function of $\mathrm{pH}$. .

A comparison of interfacial potential difference

across the PC-chol monolayer and the air/

aqueous solution interface at high and low

ionic strength. ............. 60

$172,4-D$ concentration dependence of interfacial

potential difference across the cholesterol

free GMO monolayer and air/aqueous interface. . . 62

18 The structures of isoleucine, leucine and valine

amino acid conjugates of 2,4-D......... 63

$19 \mathrm{pH}$ dependence of 2,4-D-Leu amino acid conjugate

enhanced nonactin- $\mathrm{K}^{+}$conductance of $\mathrm{PC}-\mathrm{chol}$

membranes in the low $\mathrm{pH}$ range......... 65

$20 \mathrm{pH}$ dependence of the 2,4-D-Ile amino acid conjugate

enhanced nonactin- $\mathrm{K}^{+}$conductance of $\mathrm{PC}-\mathrm{chol}$

membranes in the low pH range ........ 66

$21 \mathrm{pH}$ dependence of 2,4-D-Val amino acid conjugate

enhanced nonactin- $\mathrm{K}^{+}$conductance of $\mathrm{PC}-\mathrm{chol}$

membrane in the low pH range. . . . . . . . 
$22 \mathrm{pH}$ dependence of 2,4-D-Leu amino acid conjugate enhanced nonactin- $\mathrm{K}^{+}$conductance of PC-chol membrane in the high $\mathrm{pH}$ range. . . . . . . 68

$23 \mathrm{pH}$ dependence of 2,4-D-Ile amino acid conjugate enhanced nonactin- $\mathrm{K}^{+}$conductance of $\mathrm{PC}-\mathrm{chol}$ membranes in the high $\mathrm{pH}$ range . . . . . . 69

$24 \mathrm{pH}$ dependence of 2,4-D-Val amino acid conjugate enhanced nonactin- $\mathrm{K}^{+}$conductance of $\mathrm{PC}-\mathrm{chol}$ membranes in the high $\mathrm{pH}$ range .. . . . . . 70 Effect of $\mathrm{pH}$ on the 2,4-DCP enhanced nonactin- $\mathrm{K}^{+}$ conductance................ 71

26 The structure of indole acetic acid....... . 72 $\mathrm{pH}$ dependence of IAA enhanced nonactin $-\mathrm{K}^{+}$ conductance of PC-chol membranes . . . . . . 73

28 The structures of $2,4,5-\mathrm{T}, 2,4,6-\mathrm{T}, 2,4-\mathrm{DB}$ and 2,4-D-isobutyl ester $(2,4-D-I B E)$. . . . . 75 $\mathrm{pH}$ dependence of 2,4-DB enhanced nonactin- $\mathrm{K}^{+}$ conductance. . . . . . . . . . . . 77

$30 \quad \mathrm{pH}$ dependence of $2,4,5-\mathrm{T}$ enhanced nonactin- $\mathrm{K}^{+}$ conductance. . . . . . . . . . . . . 78

31 Effect of $2,4,5-\mathrm{T}$ and $2,4,6-\mathrm{T}$ on the nonactin- $\mathrm{K}^{+}$ conductance. . . . . . . . . . . . 79

32 Dependence of kinetic parameter $A$ on the potassium ion concentration for three concentration levels

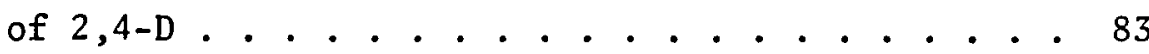


A comparison of 2,4-D concentration dependence of $\mathrm{TPhAs}^{+}$conductance with that of kinetic parameter A for PC-chol and GMO membranes. . . . 88 A comparison of ionic strength dependence of $\mathrm{TPhAs}^{+}$conductance with that of parameter A at fixed 2,4-D concentration . . . . . . . 91 Dependence of parameter $B$ on 2,4-D concentration for the switch-ON and switch-OFF transient processes. . . . . . . . . . . 93

36 A comparison of 2,4-D concentration dependence of switch-ON and switch-OFF $\mathrm{TPhB}^{-}$membrane conductance with the 2,4-D concentration dependence of the density of surface charge due to $\mathrm{TPhB}^{-}$ions adsorbed at the membrane surface. . . . . . . . . . . . . 97

37 A plot of the dependence of zero voltage relaxation time constant for $\mathrm{TPhB}^{-}$on 2,4-D concentration. . . . . . . . . . 98

38 Change of electric potential difference between membrane interior and the aqueous solution and between the membrane interior and the reaction plane as a function of 2,4-D concentration . . . 101 
Changes of electric potential difference as a function of aqueous 2,4-D concentration determined from the studies of relaxation of $\mathrm{TPhB}^{-}$conduction current in $\mathrm{PC}-\mathrm{chol}$ membranes. ............... 103

A comparison of the changes of the electric potential difference between the central plane of the membrane and the aqueous solution as determined from the changes of membrane conductance due to $\mathrm{TPhAs}^{+}$and $\mathrm{TPhB}^{-}$ions . . . . 104 A comparison of the changes of the electric potential difference between the central plane of the membrane and the adsorptionreaction plane as determined from the changes of kinetics of nonactin- $\mathrm{K}^{+}$transport and the relaxation time constant of $\mathrm{TPhB}^{-}$ transport. . . . . . . . . . . . . 106

A diagram depicting the dipole hypothesis used to interpret the effect of 2,4-D on ion transport. . . . . . . . . . . . . 10 Dependence of the kinetic parameter A for PCchol membranes on 2,4,5-T concentration. . . . . 118 
CHAPTER I

INTRODUCTION

\section{1a. BIOMEMBRANES AND ARTIFICIAL LIPID BILAYER MEMBRANES}

Membranes are essential building elements in the construction of all cells. Every cell has a limiting membrane, the plasma membrane, that separates the cell's own substance from its environment. In addition, all eukaryotic cells use a variety of differentiated intracellular membranes to construct distinct compartments within their bodies, such as nuclei, endoplasmic reticula, mitrochondria, chloroplasts, lysosomes and vesicles. Irrespective of their location, cellular membranes are essentially diffusion barriers for ions and water soluble molecules. This work is concerned with the effect of pesticides and other biologically active substances on ionic permeability of membranes.

The structure and composition of all biological membranes appear to be unique. They are about $100 \AA$ thick and consist of mainly lipids and proteins $(1,2)$. A variety of lipids is found in biological membranes (pnospholipids, sterols and sphingolipids). These structures have both polar and nonpolar regions and thus they are very well suited to form bilayer membranes because the hydrocarbon chains do not mix well with water, and so are hydrophobic. The charged head groups can reduce their electrostatic energy by associating with water, and so are called hydrophilic. Results of the studies of X-ray diffraction (3), 
differential thermal calorimetry $(4,5)$, electron microscopy $(6)$, and nuclear magnetic resonance spectroscopy (7), indicate that lipid molecules of all biomembranes are in the state of a bimolecular layer. In biomembranes, the lipid bilayer is modified by a variety of specific proteins. These can be intrinsic or integral proteins which extend completely across the membrane or periferal proteins which are loosely attached to the membrane surface. These specific proteins could be regarded as permeability modifiers of either the passive or active type. Due to physico-chemical activity of proteins the biomembranes can create and maintain chemical and electric potential gradients between the cell and its environment, as well as among different intracellular compartments. Existence of such gradients is crucial for practically all important cell activities. Modification of such gradients due to alterations in membrane permeability characteristics could obviously result in improper functioning of the cell.

The present dissertation is focused on the physical processes leading to modification of membrane ionic permeability by a certain group of biologically active substances.

Artificial bilayer membranes have been successfully used as models in the study of various properties of biological membranes. Their structures resemble those of the lipid matrix in biological membranes, especially in thickness and dielectric constant. For example, the capacitance of the artificial membranes is about $5 \times 10^{-7}$ farad $\mathrm{cm}^{-2}$ (8) which is comparable to biological membrane capacitance (9). The resistance of unmodified artificial membrane is of the order of $10^{8}$ 
ohms $\mathrm{cm}^{-2}$ (10) which is 5-7 orders of magnitude higher than that of nerve (11) but comparable to that of mitochondria (12). However, by various chemical modifiers the resistance of artificial membranes can be reduced and made comparable to that of biomembranes.

Planar bilayer membranes formed on a small hole in a thin teflon sheet separating two aqueous solutions are well suited for electrical measurements. These measurements allow a more direct investigation of the mechanism of alteration of charge transport properties of such lipid structures in the presence of other substances.

1b. BIOLOGICAL ACTIVITY OF PHENOXY PESTICIDES AND OF OTHER RELATED SUBSTANCES

Chlorinated phenoxy acids: 2,4-dichlorophenoxyacetic acid (2,4-D), 2,4-dichlorophenoxybutyric acid $(2,4-D B)$ and 2,4,5-trichlorophenoxy-acetic acid $(2,4,5-T)$ are widely used herbicides in agriculture. These agents have been known to act as uncouplers of oxidative phosphorylation (13) and plant growth regulators (14). These compounds are known to enhance nuclear protein phosphorylation in plants (15) and increase protein content in liver of rats (16). 2,4-D is toxic to animals as well as to humans (17). Development of spontaneous oscillations in transmembrane potential (repetitive action potential) and partial depolarization in the muscle tissue in the presence of $2,4-D$ (18) and redistribution of calcium in muscle at prolonged exposure have been observed (19). These observations along with the results of studies on plants have led us to investigate the possibility that 2,4-D and other phenoxy pesticides can modify the distribution of ions across membranes existing under 
normal living conditions. If the hypothesized modification of ion transport is associated with the changes of transport properties of lipid bilayer, rather than with specific interactions of pesticides and proteins, it is to be expected that their effect on ion transport can be observed and studied by measuring electrical conductivity of artificial lipid membranes. Since these phenoxy acids at low concentrations have the properties of growth regulators (auxins), a natural growth regulator indole acetic acid (IAA) also was selected for studying the effects on ion transport properties of membranes. Auxin activity of plants may also be related with the changes in ion transport across the membranes, since it has been observed that the auxin induced elongation of plants is associated with secretion of $\mathrm{H}^{+}$ions $(20,21)$.

In recent years, the metabolism of 2,4-D has been studied rather extensively in plants and plant cissue cultures $(22-28)$. It has been shown that 2,4-D is metabolized into amino acid conjugates and ring hydroxylated derivatives. The hydroxylated metabolites have been found to be physiologically inactive, and their appearance is associated with the detoxification process $(24,28)$. In contrast, the amino acid conjugates possess growth stimulation activity, especially the less polar conjugates such as with leucine, isoleucine, valine, alanine and methionine $(25,27)$. Therefore, a few of these less polar amino acid conjugates of 2,4-D were also included in the category of compounds which are our subject of interest. 


\section{CIIAPTER II}

\section{GENERAL CONSIDERATIONS REGARDING THE MECHANISM OF ELECTRICAL CONDUCTIVITY OF LIPID BILAYER MEMBRANES IN THE PRESENCE OF PHENOXY PESTICIDES AND THEIR DERIVATIVES}

In this section, several possible mechanisms by which the conductivity of lipid membranes can be altered by phenoxy pesticides will be considered. These possibilities have been taken into consideration in designing the experiments to investigate the effect of phenoxy pesticides and of other biologically active compounds on bilayer lipid membranes.

\section{2a. DIFFUSION OF ANIONS}

Diffusion of ionized molecules across membranes can be considered as one of the possible transport modes, because the chlorine substituents in these phenoxy acids may produce high solubility of these compounds at a membrane surface. This mechanism of transport would be similar to that observed for lipid soluble ions such as dipicrylamine, tetraphenylborate and tetraphenylarsonium (29-42).

The direct ion transport involves adsorption of ions within the membrane interface region, translocation across the membrane core, and desorption on the opposite side of the membrane (31). This mode of transport can be easily recognized by characteristic current or voltage transients, if the rates of adsorption and desorption of ions are small compared to the rate of electrodiffusion across the membrane interior; 
this is often the case for anions in lipid membranes. The characteristics of these transients provide useful information on the density of membrane permeable ions adsorbed at the membrane surface, as well as on the rate constant of ion translocation across the membrane interior $(29-42)$.

2b. "UNCOUPLER-TYPE" OF MEMBRANE ELECTRICAL CONDUCTIVITY INDUCED BY PHENOXY ACIDS

The ability of phenoxy acids to uncouple oxidative phosphorylation in mitrochondria (13) lends support to the hypothesis that the effect of phenoxy acids on membrane electrical conductivity can be similar to that found for other uncouplers of oxidative phosphorylation (43-47). The uncouplers make the membrane conductive by indirect transfer of hydrogen ions. Although two classes of weak acid uncouplers have been recognized and characterized by their electrical effects in bilayer membranes, the typical feature of these substances is the bell-shaped dependence of membrane conductance on $\mathrm{pH}$, which is due to the coexistence of diffusion of the neutral uncoupler molecules across the membrane in one direction, and electrodiffusion of the negatively charged uncoupler ion or dimer complex in the opposite direction (48). This mechanism is of great biological significance since these uncouplers can dissipate hydrogen ion concentration gradients and electrical potential differences across membranes. High efficiency of these dissipative mechanisms originate from the fact that both ionized and the neutral uncoupler molecules have to cross only the nonpolar membrane core. Thus, these species have to diffuse in the membrane over a very short distance. 
In the aqueous phase the flow of electric charge is maintained by protons, which can propagate faster than any other ions.

\section{2c. MEDIATED CATION TRANSPORT}

Another conceivable mode of action of chlorinated phenoxy acids is the mediation of cation transport and cation-proton exchange, similar to that observed for nigericin (49). This hypothesis is based on common structural features and similarity in biological action: both nigericin and phenoxy acids contain one carboxylic group, they both have hydrophobic and hydrophylic regions within the molecule, and both are known to be uncouplers of oxidative phosphorylation $(13,50)$. It has been proposed that the nigericin-induced electrical conductivity in bilayer membranes is due to a charged, membrane permeable complex. Toro et al (51) have suggested that such a complex is formed between two nigericin molecules and one potassium ion, whereas Markin et al. (52) prefer to interpret their experimental results in lerms of a negatively charged membrane permeable complex of the $\mathrm{KA}_{2}^{-}$and $\mathrm{HA}_{2}^{-}$types.

2d. MODIFICATION OF MEMBRANE SELECTIVITY AND CHANGES OF KINETICS OF CARRIER-MEDIATED ION TRANSPORT IN THE PRESENCE OF PHENOXY PESTICIDES

In addition to the direct mechanism of electrical conductivity induced by permeation of electrically charged phenoxy acid molecules or its complexes, the mechanisms in which phenoxy acids affect the kinetics of transfer of other membrane permeable ions is considered. It is possible, for example, that when phenoxy acids are present in the 
membrane, the membrane thickness or the ion diffusion constant changes; these effects would affect positively and negatively charged ions more or less equally. Our special interest, however, is in the mechanism by which membrane selectivity to ions can be altered when "impurities" such as 2,4-D and other phenoxy acids are present in the membrane environment. The ion selectivity modification can be achieved if the agent selectively influences either the density of membrane permeable ions at the membrane surface, or the probability of ion translocation across the membrane interior. Such phenomena are well known. For example chaotropic ions (53), or ions of dinitrophenol $(54,55)$ or chlorinated phenol (56) adsorb at the membrane surface and modify the density of permeable ions in the membrane. In these examples the selectivity modification is associated with the formation of an electrical double layer extending from the membrane surface into the aqueous solution. Another mode of action of selectivity modifiers can be associated with the change of dipole potentials at the membrane/water interface. It is well established that membrane associated dipoles play an important role in ion transport, and that intrinsic membrane dipoles in the boundary region affect membrane selectivity. For example, the difference in ionic selectivity characterized by ratios of cationic and anionic conductances of glyceride and phospholipid membranes has been related to the existence of dipolar potential differences of membrane interfacial regions (57-59). Szabo (60-62) and others (63-65) have demonstrated that the increase of cholesterol content in glycerolmonooleate membranes increases the permeability coefficients of negative ions, whereas the permeability coefficients of positive ions 
decreases. The effect of cholesterol is largely due to the increase of electrostatic potential of the membrane interior. Membrane conductivity changes observed in the presence of phloretin (66-69), salicylamide (70), as well as of some nitrophenols and acetophenons (66) have been also associated with the changes of interfacial dipolar potentials.

The change of distribution of the electric potential within the membrane due to the change of interfacial dipole moment can result in the change of kinetics of carrier-mediated transport $(62-64,66,67)$. The interference of pesticides with the carrier-mediated transport of ions is of current interest since this type of transport is widely utilized in biological membranes. The action of toxic substances on membranes is often very complex, even in the case of simple systems, such as artificial lipid bilayers. For example, TTFB and other structurally related benzimidazoles not only induce electrical conductivity in membranes by themselves $(47,71,72)$, but as Kuo et al. found $(73,74)$, they can also block valinomycin-mediated transport of potassium. 


\section{CHAPTER III}

\section{THEORETICAL CONCEPTS OF ION TRANSPORT IN LIPID BILAYER}

\section{3a. ENERGY BARRIERS AND ION TRANSPORT}

In the following section we consider the factors which determine the transfer of ions across the interior of membranes in response to an applied electric potential difference. We will first discuss the nature of the energy barrier that membranes present to ions and then the possible approaches for the treatment of ion translocation across lipid membranes.

\section{Energy Barriers}

The barrier properties of a lipid bilayer are primarily associated with the low concentration of ions within the membrane. This low concentration of ions is due to the large electrostatic charging energy (work) required to remove an ion from aqueous phase, which has a high dielectric constant $\left(\varepsilon_{w} \simeq 80\right)$ and electrolyte content, and insert it into a less polarizable hydrocarbon phase of the membrane interior with a lower dielectric constant $\left(\varepsilon_{m} \simeq 2-3\right)$. If the finite thickness of the membrane is ignored, the membrane can be treated as a homogeneous phase. In such a case this work can be easily calculated, and it is given by the Born charging energy, $W_{B}(r)$ :

$$
W_{B}(r)=\frac{z^{2} e^{2}}{8 \pi \varepsilon_{0} r}\left[\frac{1}{\varepsilon_{m}}-\frac{1}{\varepsilon_{w}}\right]
$$


where $z$ is the valence of the charged particle, $e$ is the elementary charge, $\varepsilon_{m}$ is the dielectric constant of the hydrocarbon phase of the membrane, $\varepsilon_{w}$ is the dielectric constant of water, and $r$ is the radius of the particle. This work will determine the distribution of ions between the hydrocarbon phase and water. The partition coefficent, $K$, is essentially a Boltzmann factor,

$$
K=\frac{c_{m}}{c}=\exp \left(\frac{-W_{B}(r)}{k T}\right)
$$

where $c_{m}$ and $c$ are the concentration of ions in the interior of the membrane and in the aqueous phase respectively. For an univalent ion with radius $r=0.2 \mathrm{~nm}$, we obtain $W_{B}(r)=70 \mathrm{kT}$ and $K=$ $4 \times 10^{-31}$. On the other hand for a larger ion, $r=0.4 \mathrm{~nm}, W_{B}(r)=$ $35 \mathrm{kT}$ and $\mathrm{K}=6 \times 10^{-16}$. Thus, doubling of ion radius has increased partition coefficient by factor of $10^{15}$. This simple model is useful as it explains why lipid membranes are almost impermeable to small ions. However, it is not realistic to consider a membrane with a thickness of $5-10 \mathrm{~nm}$ as a macroscopic phase, since the electrostatic field around the ion induces large polarization charges at the membrane solution interfaces. Therefore, a better approach for the calculation of electrostatic potential energy barrier of an inn is to perform the complete image charge calculation considerairg the two membrdne/water interfaces. The shape of the image charge barrier for ar univalent ion of radius $0.2 \mathrm{~nm}$ as a function of dielectric constant of the membrane interior calculated by Neumcke and Lauger (75) is shown in Fig. 1 . 


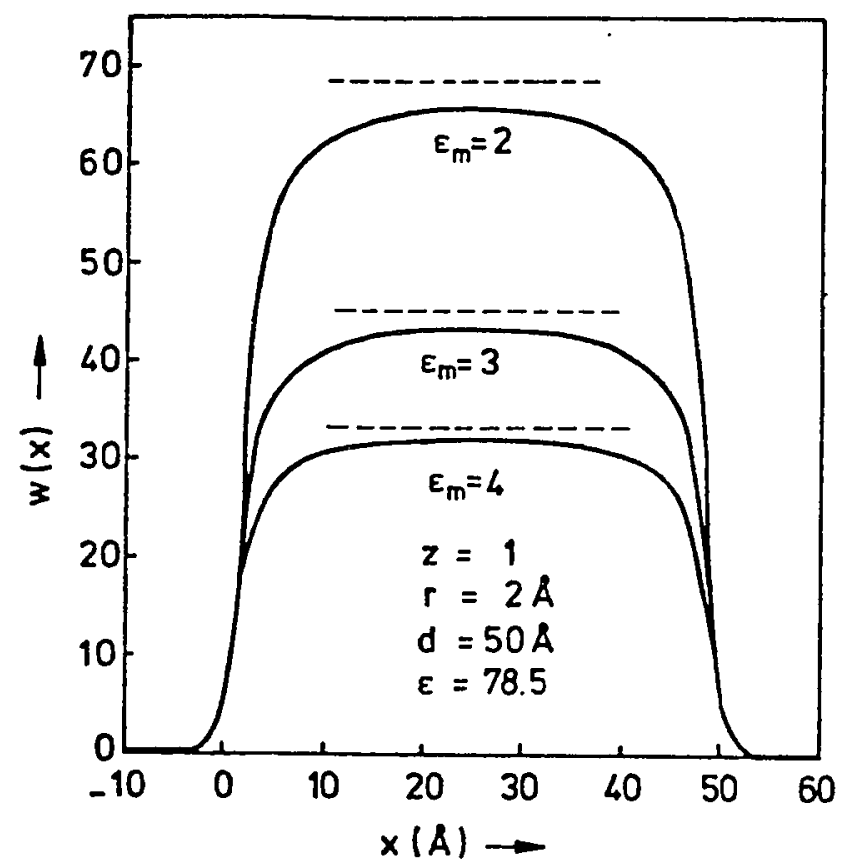

Figure 1. The "image charge" barrier experienced by an ion crossing a bilayer membrane. $z$ is the valence of the ion, $r$, the radius, $d$ is the thickness of the membrane, $\varepsilon_{m}$ and $\varepsilon$ are dielectric constants of the membrane and water respectively. $W(x)$ is in units of $k T$. The dashed lines are the Born charging energies corresponding to an ion in the membrane of dielectric constant $\varepsilon_{\mathrm{m}}$. This figure was taken from the reference 75 . 
The total energy barrier for an ion includes not only the image potential, but also the hydrophobic energy due to the interaction of the ion and the hydrocarbon interior of the membrane. This hydrophobic energy $W_{h}(x)$ is negative. As a result of superposition of the hydrophobic and electrostatic potential energy profiles of molecular ions, potential energy minimas exist at the hydrocarbon side of the membrane/water interfaces and are separated by a broader barrier in the center of the membrane $(35,76)$.

Up to now the membrane has been treated simply as a thin hydrocarbon film interposed between two aqueous solutions. In reality, however, the potential energy of an ion in the membrane is influenced by the structure of the polar layer at the membrane surface, which is formed by the hydrophilic head groups of the lipid molecules. If the head groups bear net electric charge, there is an additional potential difference between the membrane surface and the aqueous solution. For example, membranes formed by phosphatidylglycerol, phosphatidylserine and phosphatidylinositol contain net negative charge at the membrane water interface, while the neutral lipids GMO, GDO and zwitterionic lipid phosphatidylcholine (PC) and phosphatidylethanolamine (PE) do not possess a surface charge in a wide $\mathrm{pH}$ range. This surface charge will affect the adsorption of molecular ions on the membrane surface. The equilibrium distribution of ions between the membrane surface and the bulk aqueous phase depends on the ratio e $\psi_{s} / k T . \psi_{s}$ is the surface potential which originates from the surface charge at the membrane surface and is usually determined by the Gouy-Chapman theory of the diffuse double layer. It has been shown that the Gouy-Chapman theory 
provides an adequate description of the relationship between $\psi_{S}$ and the surface charge density $(55,70,77-80)$.

Even if the net charge of the head group vanishes, an electric potential drop may exist in the interfacial region due to the dipolar layers associated with the head groups of lipid molecules constituting the membrane. Unfortunately, little is known about the orientation of the dipoles at the membrane/solution interface. The magnitude of the dipole potential can be estimated from surface potential measuremnts at the air/water interface and air/lipid monolayer/water interface $(58,59$, 81-84) and also directly in lipid bilayers $(35,85)$. The results suggest that the interior of lipid bilayer has a large positive potential relative to the aqueous phases. This potential results in a strong intrinsic membrane selectivity for anions. The changes of the dipolar potential difference will lead to changes in anion and cation conductances. In fact, the conductance of a lipid soluble anion $\mathrm{TPhB}^{-}$is about 6 orders greater than the conductance of a structurally similar cation $\operatorname{TPhAs}^{+}(35)$.

It has been show that the ionic permeability of a lipid bilayer is determined by the height and the shape of the potential energy barrier for the molecular ion. The shape of the barrier in the middle of the membrane is determined by the sum of image-force potential energy $W_{i}(x)$ and the hydrophobic potential energy $W_{h}(x)$. The absolute height of the barrier in the middle of the membrane will be determined by $W_{i}(x)$ and $W_{h}(x)$ as well as by the electrostatic potential difference between the bulk aqueous phase and the membrane surface, due to the presence of surface charges and oriented dipoles. 
Ion Transport

For treating the ion transport across the membrane, two theories are currently being used: the Nernst-Planck analysis and the Eyring rate theory developed for the description of kinetics of chemical reactions. The Nernst-Planck analysis is based on the description of diffusion of ions across the membrane potential energy barrier in the presence of an applied electric field. In Eyring rate theory, the transport of an ion through the membrane is treated as hopping over a series of activation energy barriers $(86,87)$. In this work, we will use the Nernst-Planck formalism to describe the transmembrane ion transport.

The ion flux $\Phi$ is given by

$$
\Phi=-\frac{D}{k T} c \frac{d \mu}{d x}
$$

where $D$ is the diffusion coefficient of the ion, $c$ the ion concentration, $\mu$ the ion electrochemical potential and $x$ the coordinate normal to the membrane solution interface. The electrochemical potential $\mu$ is the sum of chemical potential and the electric potential energy of the ion due to electrical potential $\psi$

$$
\mu=\mu_{0}+k T \ln c+z e \psi
$$

$\mu_{0}$ being the standard chemical potential. The chemical potential is a function of $x$, since the energy barrier $W(x)$ is not constant through the membrane interior. (Apart from an additive constant, $\mu_{0}$ is equal to the work $W$ required to transfer an ion from a distant point in the aqueous phase to a point $x$ in the membrane. $\mu_{0}=w_{x}+$ constant.) For simplicity, 
the activity coefficient of the ion is assumed to be unity. The electric potential $\psi$ existing in the membrane includes the potential due to an external applied voltage, V, and also any "dipolar potential $\psi_{\mathrm{m}}$ due to the dipoles located near the membrane surface. Since there are no dipolar groups in the membrane interior, the dipolar potential $\psi_{\mathrm{m}}$ is expected to be constant in the membrane interior, and to fall off only near the membrane boundaries. This potential will only affect the total height of the potential barrier of the ion. If there is any net surface charge, a surface potential $\psi_{\mathbf{S}}$ should, in principle, also be included in $\psi$. However, $\psi_{S}$ is usually associated with boundary conditions and determines the concentration of ions at the membrane surface via the Boltzmann factor $\exp \left(-\right.$ ez $\left.\psi_{s} / k T\right)$. Since the primary purpose of Eq. 3 is to describe the flux of ions in the membrane, the electric potential of the membrane surface is usually excluded from the term (ze $\psi$ ) of Eq. 4 .

The stationary flux of ions can be obtained by integrating the Eq. 3. It can be shown that it is equal to

$$
\begin{aligned}
\phi & =\frac{c^{\prime} \exp (-z e V / 2 k T)-c^{\prime \prime} \exp (z e V / 2 k T)}{\exp (-z e V / 2 k T) \int_{0}^{d} \frac{\exp (W(x) / k T+z e \psi / k T)}{D}} d x \\
& =k_{i}^{\prime} c^{\prime}-k_{i}^{\prime \prime} c^{\prime \prime}
\end{aligned}
$$

where $c^{\prime}$ and $c^{\prime \prime}$ are the concentrations of the ions at left and right membrane interface. $k_{i}^{\prime}$ and $k_{i}^{\prime \prime}$ are the rate constants for ions 
crossing the membrane from left to right and right to left. 1 In the limit of zero applied voltage, the translocation rate constant $k_{i}$ reaches a limiting value;

$$
k_{i}=\frac{1}{\int_{0}^{d} \frac{\exp \left(W(x) / k T+z e \psi_{m} / k T\right)}{D}} d x
$$

and can be approximated by,

$$
k_{i} \approx \frac{\exp \left(-z e \psi_{m} / k T\right)}{\int_{0}^{d} \frac{\exp (W(x) / k T)}{D}} d x
$$

because $\psi_{m}$ is approximately constant near the middle section of the membrane. Eq. 8 has a great experimental significance in that it provides a relationship between the rate constant of ion translocation across the membrane, $k_{i}$, and the dipolar potential difference of the membrane/ water interface $\psi_{\mathrm{m}}$. Therefore, the dipolar potential difference changes can be experimentally determined from the changes of ion translocation rate constant $k_{i} \cdot{ }^{2}$

${ }^{1}$ If the adsorption planes are not exactly at the interfaces, $\quad(x=0$ and d) but at $x=n$ and $d-n$, the integration should be done from $n$ to $d-n$ instead of 0 and $d$.

${ }^{2}$ If the location of adsorption planes is at $x=n$ and $d-n$, the dipole potential could affect the adsorption of ions (i.e. affect the partition coefficient). In this case a fraction of dipole field will affect the partition of ions which could be accounted for by changing the concentration of ions in membrane by the Boltzmann factor. The rest of the dipolar field will affect the rate constant of ion transport across the membrane as discussed. 
3b. THE CARRIER MODEL FOR NONACTIN-MEDIATED $\mathrm{K}^{+}$TRANSPORT

The transport scheme for nonactin-mediated $\mathrm{K}^{+}$transport is well understood and can be described by a kinetic model of carrier transport $(81,88-91)$ (Fig. 2). The transfer of $\mathrm{K}^{+}$ion across the membrane

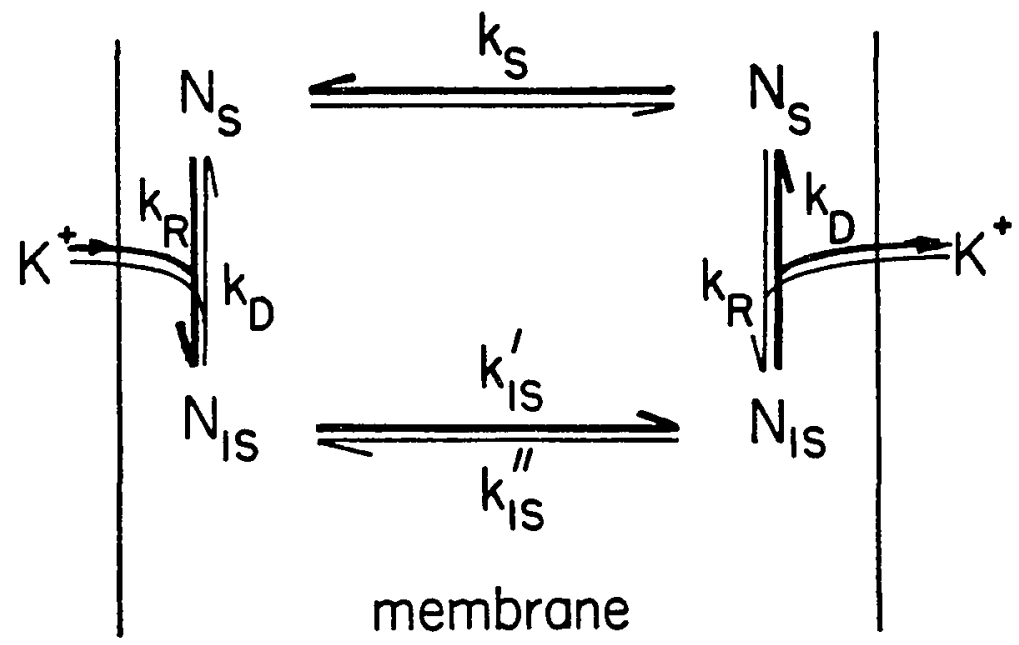

Figure 2. Mechanism of potassium ion transport across membrane by nonactin. Symbols are defined in the text.

involves several distinct processes: the formation of the membrane permeable nonactin $-\mathrm{K}^{+}$complex at the membrane/water interface (a process characterized by the complex formation rate constant, $k_{R}$ ), the electro-diffusion of the complex across the membrane (characterized by the rate constant of complex translocation, $\mathrm{k}_{\mathrm{IS}}$ ), the release of potassium ion from the carrier (rate constant of complex dissociation, $k_{D}$ ), and the back diffusion of neutral carrier (rate constant of back diffusion, $k_{s}$ ). The only type of ion in the membrane is the nonactin$\mathrm{K}^{+}$complex. Thus, the current density $j$ is given by (Eq. 6) 


$$
J_{I S}=e\left(k_{I S}^{\prime} N_{I S}^{\prime}-k_{I S}^{\prime \prime} N_{I S}^{\prime \prime}\right)
$$

where $N_{\text {IS }}^{\prime}$ and $N_{\text {IS }}^{\prime \prime}$ are the concentrations of the complex at the left and right interfaces and $k_{I S}^{\prime}$ and $k_{\text {IS }}^{\prime \prime}$ are the rate constant of complex translocation from left to right and right to left, respectively. In the stationary state, the membrane current density can be written as (92)

$$
J_{I S}=\left(e N_{S} c_{K^{+}}\right) \frac{k_{R}^{\prime} k_{I S}^{\prime} / k_{D}^{\prime}-k_{R}^{\prime \prime} k_{I S}^{\prime \prime} / k_{D}^{\prime \prime}}{1+k_{I S}^{\prime} / k_{D}^{\prime}+k_{I S}^{\prime \prime} / k_{D}^{\prime \prime}+\left(k_{R}^{\prime} k_{I S}^{\prime} / k_{D}^{\prime}+k_{R}^{\prime \prime} k_{I S}^{\prime \prime} / k_{D}^{\prime \prime}\right) c_{K^{+}} / 2 k_{S}} \text {. }
$$

The single and double primed quantities refer to the voltage dependent rate constants at the left and right sides of the membrane, respectively, and $i_{S}$ is the number of adsorbed neutral carrier molecules per unit of membrane surface.

Treating the membrane as an image potential barrier for the diffusing ions, the voltage dependent rate constants of translocation of the charged complex can be written in the following way (35):

$$
\begin{aligned}
& k_{I S}^{\prime}=k_{I S O} \exp \left[-\omega(e V / k T)^{2}\right] \exp (-B e V / 2 k T) \\
& k_{I S}^{\prime \prime}=k_{I S O} \exp \left[-\omega(e V / k T)^{2}\right] \exp (B e V / 2 k T)
\end{aligned}
$$

Here $V$ is the applied potential difference between the right side and the left side of the membrane, and $k_{\text {ISO }}$ is the translocation rate constant in the limit of zero applied voltage. Parameter $\beta$ is the 
fraction of applied voltage that is effective in ion translocation ${ }^{3}$ (35); and is a small constant whose value is dependent on the membrane thickness (35). Consistent with the barrier model is the assumption that $(1-\beta) / 2$ is the fraction of the applied potential difference between the recombination plane and the aqueous solution so that the recombination and dissociation rate constants are voltage dependent as well, i.e.,

$$
\begin{aligned}
& \mathrm{k}_{\mathrm{R}}^{\prime}=\mathrm{k}_{\mathrm{RO}} \exp [-(1-\beta) \mathrm{eV} / 4 \mathrm{kT}], \quad \mathrm{k}_{\mathrm{R}}^{\prime \prime}=\mathrm{k}_{\mathrm{R} 0} \exp [(1-\beta) \mathrm{eV} / 4 \mathrm{kT}] \\
& \mathrm{k}_{\mathrm{D}}^{\prime}=\mathrm{k}_{\mathrm{DO}}-\exp [(1-\beta) \mathrm{eV} / 4 \mathrm{kT}], \quad \mathrm{k}_{\mathrm{D}}^{\prime \prime}=\mathrm{k}_{\mathrm{DO}} \exp [-(1-\beta) \mathrm{eV} / 4 \mathrm{kT}] .
\end{aligned}
$$

The value of parameter $B$ is not a known a priori, but there are good arguments that it should be close to unity $(35,65)$. Hladky's results for nonactin and trinactin complexes in GMO/hexadecane membranes suggest that $\beta$ is between 0.8 and $0.9(90,91)$; these references should also be consulted for more detailed discussion of the problem of voltage dependent rate constants. In order to reduce the number of unknown parameters, $B$ is assumed to be 1 so that $k_{R}^{\prime}=k_{R}^{\prime \prime}=k_{R}$ and $k_{D}^{\prime}=k_{D}^{\prime \prime}=k_{D}$. The membrane conductance $G(V)=J_{I S} / V$ can be obtained by substituting Eqs. 10 and 11 into Eq. 9. giving

$G(V)=\frac{1}{V} \frac{\left(2 e k_{R} N_{S} k_{I S O} c_{K^{+}} / k_{D}\right) \exp \left[-\omega(e V / k T)^{2}\right] \sinh (e V / 2 k T)}{1+\left(2 k_{I S O} / k_{D}+k_{I S O} k_{R}{ }_{K^{+}} / k_{S} k_{D}\right) \exp \left[-\omega(e V / k T)^{2}\right] \cosh (e V / 2 k T)}$.

${ }^{3}$ If the adsorption planes for the nonactin- $\mathrm{K}^{+}$complex are not at the membrane/water interface (i.e. at $x=0$ and d) but at a distance $n$ from the interfaces, only a fraction of the externally applied voltage will be used to drive the ions across the membrane from one adsorption plane to the other. 
The normalized membrane conductance is given by

$$
\frac{G(V)}{G(0)}=\frac{2 k T}{e V} \frac{(1+A) \exp \left[-\omega(e V / k T)^{2}\right] \sinh (e V / k T)}{1+A \exp \left[-\omega(e V / k T)^{2}\right] \cosh (e V / 2 k T)}
$$

where $G(0)$ is the zero voltage conductance, and parameter A involving two combinations of rate constants, is equal to

$$
A=2 k_{I S O} / k_{D}+k_{I S O} k_{R^{C}}{ }^{+} / k_{S} k_{D}
$$

As follows from the model (Eqs. 9, 12 and 13) there are two processes limiting the flow of $\mathrm{K}^{+}$ions across the membrane in the presence of 2,4-D: (1) a slow rate of recombination of carriers with

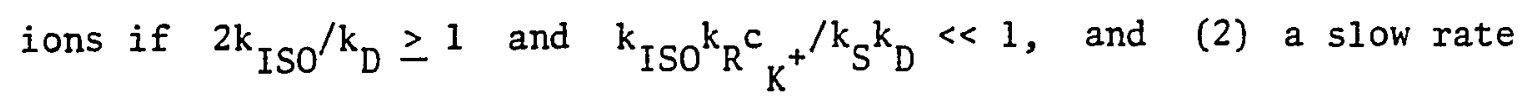
of back diffusion of unloaded carrier if $\mathrm{k}_{\mathrm{ISO}} \mathrm{k}_{\mathrm{R}}{ }^{\mathrm{C}} \mathrm{K}^{+} / \mathrm{k}_{\mathrm{S}} \mathrm{k}_{\mathrm{D}} \geq 1$ and $2 \mathrm{k}_{\mathrm{ISO}} / \mathrm{k}_{\mathrm{D}} \ll 1$. The latter process depends upon the potassium ion concentration.

\section{3c. TRANSPORT OF TPhAS ${ }^{+}$IONS ACROSS THE MEMBRANE}

Tetraphenylarsonium $\left(\mathrm{TPhAs}^{+}\right.$) is a lipid soluble cation. Thus, in the presence of externally applied potential there will be a net flow of these ions across the membrane. The ion translocation involves the following processes: (a) diffusion of the ion in the aqueous phase to the left membrane interface (adsorption) (b) transmembrane ion translocation and (c) desorption of the ion at the right interface and diffusion into right aqueous phase. Since the membrane translocation rate constant is considerably smaller compared to that of the negative ions (due to the positive dipole potential in the membrane interior), the diffusion flux of $\mathrm{TPhAs}^{+}$ions in the aqueous medium can 
be significantly greater than the maximum possible flux of ions across the membrane. This means that the ion transport is limited by the membrane and not by the diffusion across unstirred layers adjacent to the membrane.

Using the Nernst-Planck electrodiffusion equation and treating the membrane as an image potential barrier, for equal concentrations of TPhAs $^{+}$ions in both aqueous phases the steady state current can be written as

$$
J=2 e c k k_{i} \exp \left[-\omega(e V / k T)^{2}\right] \sinh (B e V / 2 k T)
$$

where $c$ is the concentration of $\mathrm{TPhAs}^{+}$ions in the aqueous phase, $K$ is the partition coefficient of the ion between the aqueous phase and membrane, and $k_{i}$ the voltage independent translocation rate constant of the ion across the membrane.

\section{3d. TRANSPORT OF TETRAPHENYLBORATE IONS ACROSS LIPID MEMBRANES}

The usefulness of $\mathrm{TPhB}^{-}$as a membrane probe originates from the high coefficient of distribution of $\mathrm{TPhB}^{-}$ions between the membrane surface and water, and from the experimentally favorable redistribution time of $\mathrm{TPhB}^{-}$ions in membranes $\left(10^{-2}-10^{-3} \mathrm{~s}\right)$. Current relaxation experiments with $\mathrm{TPhB}^{-}$permit one to measure separately both the density of $\mathrm{TPhB}^{-}$adsorbed at the membrane surface, and the time constant of the translocation of ions from one interface to the other $(31,33-35,40)$. Immediately after the application of an electric potential difference step across the membrane, an exponentially decaying transient conduction current is observed due to the flow of $\mathrm{TPhB}^{-}$ions across the 
membrane interior from ion potential well at one interface to that at the other (31). The diffusion of $\mathrm{TPhB}^{-}$ions toward and away from the membrane is insignificant due to the fact that the TPhB permeability of membranes is much greater than that of the aqueous solution. Thus, on the time scale of the current decay the amount of $\mathrm{TPhB}^{-}$ions at the membrane surface is conserved, i.e., $N^{\prime}+N^{\prime \prime}=2 N_{e q}$ where $N^{\prime}$ and $N^{\prime \prime}$ are the surface concentrations of the ions at left and right energy minimas. $N=\delta \mathrm{c}$, where $\mathrm{c}$ is the volume concentration of ions in the membrane and $\delta$ is the thickness of the adsorption layer.

Although the boundary concentrations change with time, one can assume the transport through the membrane interior is already at steady state $(32,35)$. After the displacement current has decayed, the pseudostationary transmembrane current density $J(V, t)$ can be written as

$$
J(V, T)=-e \frac{k_{i}(V)}{\delta}\left[N^{\prime \prime} \exp (-\beta e V / 2 k T)-N^{\prime} \exp (\beta e V / 2 k T)\right]
$$

where $k_{i}(V)$ is the translocation rate constant which can be obtained by treating the membrane as an image potential energy barrier in the following form

$$
k_{i}(V)=\frac{\exp \left[-\omega(e V / k T)^{2}\right] D_{m}}{\int_{\eta}^{d-\eta} \exp \left[W^{\prime}(x) / k T\right] d x}=k(0) \exp \left[-\omega(e V / k T)^{2}\right] .
$$

$D_{m}$ is the ion diffusion coefficient in the membrane interior. The membrane is assumed to extend from $x=0$ to $x=d$; the $\mathrm{TPhB}^{-}$adsorption planes are at $\eta$ and $d-n . W^{\prime}(x)$ is the electrostatic potential energy of the ion at a distance $x$ with respect to that at $x=n$. $w$ is a coefficient taking into account the deformation of the ion potential energy barrier due to the applied potential difference $V$ 
and $B$ is the fraction of the applied voltage between the adsorption minimas located at $\eta$ and $d-\eta$.

Since all the ions adsorbed onto the bilayer are located in two boundary regions, the rate at which its concentration changes at each surface is equal to the number of ions translocated

$$
\begin{aligned}
& N^{\prime}(t)=N_{\text {eq }}-\int_{0}^{t} J(V, \xi) d \xi \\
& N^{\prime \prime}(t)=N_{\text {eq }}+\int_{0}^{t} J(V, \xi) d \xi
\end{aligned}
$$

where $\mathrm{N}_{\mathrm{eq}}$ is the equilibrium surface concentration before the application of the voltage pulse.

Substituting Eq. 18 into Eq. 16 and solving for the pseudostationary current density, it can be shown that

$$
J(V, t)=\left.J(V)\right|_{t=0} \exp (-t / \tau)
$$

where $\left.J(V)\right|_{t=0}$ is the initial current density obtained by extrapolating the conduction current density to zero time.

The initial membrane conductance $\left.G^{O N}(V)\right|_{t=0}=\left.J^{O N}(V)\right|_{t=0} / V$ is equal to

$$
\begin{aligned}
\left.G^{O N}(V)\right|_{t=0} & =2 e \frac{N_{e q}}{\delta} k_{i}(V) \sinh (e \beta V / 2 k T) \\
& =\left.\frac{2 k T}{e B V} \frac{\sinh (e \beta V / 2 k T)}{\exp \left[\omega(e V / k T)^{2}\right]} G^{O N}(0)\right|_{t=0}
\end{aligned}
$$

where $\left.G^{O N}(0)\right|_{t=0}$ is the zero voltage conductance given by 


$$
\left.G^{O N}(0)\right|_{t=0}=\frac{e^{2} \beta D_{m} N_{e q} / \delta}{k T \int_{n}^{d-n} \exp \left[W^{\prime}(x) / k T\right] d x}=\frac{e^{2} \beta}{k T} \frac{N_{e q}}{\delta} k_{i}(0)
$$

The superscript ON refers to the transient process observed after the step like increase of membrane bias from zero to $V$.

After the application of the bias voltage $V$, the net ion flow in the membrane decreases exponentially with time (Eq. 19) because of the depletion of the ions on the negatively biased side of the membrane and their accumulation on the positive side. The characteristic time of this process is

$$
\begin{aligned}
\tau^{O N}(V) & =\frac{\delta}{2 k_{i}(V)} \frac{1}{\cosh (e \beta V / 2 k T)} \\
& =\frac{\exp \left[\omega(e V / k T)^{2}\right]}{\cosh (e \beta V / 2 k T)} \tau^{O N}(0)
\end{aligned}
$$

where $\tau^{O N}(0)=\frac{\delta}{2 D_{m}} \int_{n}^{d-\eta} \exp \left[W^{\prime}(x) / k T\right] d x=\frac{\delta}{2 k_{i}(0)}$.

The total amount of charge transferred across the membrane during the current relaxation process is equal to

$$
\begin{aligned}
Q^{O N}(V) & =Q_{a d s} \tanh (e \beta V / 2 k T)=\left.\int_{0}^{\infty} J^{O N}(V)\right|_{t=0} \cdot \exp \left(-t / \tau^{O N}(V)\right) d t \\
& =\left.J^{O N}(V)\right|_{t=0} \cdot \tau^{O N}(V) .
\end{aligned}
$$

where $\left.J^{O N}(V)\right|_{t=0}$ is the initial membrane conduction current density, and $\mathrm{Q}_{\mathrm{ads}}=\mathrm{eN}_{\mathrm{eq}}$ is the equilibrium density of membrane surface charge due to adsorbed $\mathrm{TPhB}^{-}$ions. 
Transient conduction currents can also be observed when the external bias voltage is switched off. These "off-currents" are caused by the return flow of $\mathrm{TPhB}^{-}$ions that have been displaced from the equilibrium distribution under the previously applied bias. The initial membrane conductance associated with the membrane conduction when the bias voltage is turned off can be defined in a similar way,

$$
\left.G^{O F F}(V)\right|_{t=0}=\left.\frac{2 k T}{e \beta V} \tanh (e \beta V / 2 k T) \cdot G^{O F F}(0)\right|_{t=0}
$$

It can be shown that $\left.G^{O F F}(0)\right|_{t=0}$, the zero voltage initial conductance of the switch-OFF process, is equal to the initial zero voltage conductance observed under the switch-ON conditions.

The time constant of the switch-OFF relaxation current is equal to

$$
\tau^{\mathrm{OFF}}=\frac{\delta}{2 \mathrm{D}_{\mathrm{m}}} \int_{n}^{\mathrm{d}-n} \exp \left[\mathrm{W}^{\prime}(\mathrm{x}) / \mathrm{kT}\right] \mathrm{dx}
$$

In contrast to the time constant of the switch-ON process, the time constant $\tau^{O F F}$ is voltage independent, and is equal to the zero voltage time constant of the switch-ON process, i.e., $\tau^{\mathrm{OFF}}=\tau^{\mathrm{ON}}(0)$.

The total amount of electric charge transferred across the membrane during the OFF-pulse charge redistribution is given by

$$
\begin{aligned}
Q^{O F F}(V) & =\left.\int_{0}^{\infty} J^{O F F}(V)\right|_{t=0} \cdot \exp \left(-t / \tau^{O F F}\right) d t \\
& =\left.J^{O F F}(V)\right|_{t=0} \cdot \tau^{O F F} .
\end{aligned}
$$

If there is no exchange of ions between the membrane and the aqueous solution, then $Q^{O F F}(V)=Q^{O N}(V)$. 
Andersen and Fuchs (35) have shown that the above described quasistationary model of electrodiffusion of $\mathrm{TPhB}^{-}$ions across the membrane represented by an image potential energy barrier satisfactorily describes the transient membrane current for low $\mathrm{TPhB}^{-}$concentrations. At high concentrations (exceeding $10^{-6} \mathrm{M} \mathrm{TPhB}^{-}$) the transport process deviates from this simple model due to the existence of large boundary potentials. Thus, considering the boundary potentials due to adsorbed $\mathrm{TPhB}^{-}$at high concentrations, "the three capacitor model" has been developed by Andersen at al. (40), which takes into account the adsorption of $\mathrm{TPhB}^{-}$on the membrane surface and the time dependence of the effective voltage driving ion translocation at high concentrations. However, this model cannot explain explicitly the increase of zero voltage time constant $\tau(0)$ with the concentration of $\mathrm{TPhB}^{-}$and also the anomalous behavior of zero voltage conductance with concentration of $\mathrm{TPhB}^{-}$. These anomalies are partially understood in that Wang and Bruner (93) have explained the increase of $\tau(0)$ at high concentrations by introducing the concept of discrete charges. In the present study, these refinements have not been taken into account, since the TPhB ${ }^{-}$ concentrations which have been employed are small enough to apply the simplest model. 


\section{CHAPTER IV}

\section{PROCEDURES AND MATERIALS}

\section{4a. MEMBRANE FORMATION AND METHODS OF PREPARATION OF SOLUTIONS}

The optically black lipid membranes were formed at room temperature by the brush technique (94) on a $2 \mathrm{~mm}$ diameter hole in a wall of a Teflon cell immersed in the aqueous solution. Two types of membranes were studied: egg lecithin/cholesterol/n-decane (PC-chol) and monoolein/ n-decane (GMO). In PC-chol membranes the cholesterol mole fraction was 0.76; the total lipid content (i.e. lecithin + cholesterol) in the membrane forming solution was $11.5 \mathrm{mg}$ per $\mathrm{ml}$. The GMO membranes were cholesterol free; the composition of the meinbrane forming solution was $25 \mathrm{mg}$ per $\mathrm{ml}$.

The aqueous solution, except as noted below, contained $\mathrm{KC} 1$, buffer (phosphate, citrate, borate, ratios $0.002 \mathrm{M} / 0.002 \mathrm{M} / 0.0005 \mathrm{M}$ ), and the compounds which are being tested; LiC 1 was used to adjust ionic strength. Tetraphenylarsonium $\left(\mathrm{TPhAs}^{+}\right.$) chloride, sodium tetraphenylborate $\left(\mathrm{TPhB}^{-}\right)$ and nonactin were first dissolved in ethanol, and then the ethanolic stock solution was added to the aqueous solution which was prepared fresh every day. The volume of ethanol in the final solution did not usually exceed $0.5 \%$. The solutions containing tetraphenylborate were potassium-free to avoid precipitation of KTPhB. Nonactin was added to the membrane forming solution in order to shorten the equilibrium time of nonactin distribution between the aqueous medium and the 
membrane. Since 2,4-D esters are not soluble in water, they were incorporated into the membrane forming solution.

The Teflon compartment of the measuring cell was made out of virgin material. It was boiled in ethanolic solution of sodium hydroxide for about 5 minutes and then washed in deionized water before each experiment. Occasionally the cell was soaked in chromic acid. Cleanliness of the system was checked periodically by measuring background conductivity of membranes (order of $10^{-8} \mathrm{~s} / \mathrm{cm}^{2}$ ) in the absence of membrane modifiers. Despite this cleaning procedure, nonactin contamination in the walls of Tefion cell was experienced. Prolonged baking of the cell at $200-270^{\circ} \mathrm{C}$ effectively removed nonactin from the Teflon.

\section{4b. STEADY STATE CONDUCTANCE MEASUREMENTS}

The current-voltage characteristics of membranes were measured at least 10-15 minutes after the membrane became black and after the conductivity stabilized. For monitoring the conduction state of membrane, a voltage pulse of $25 \mathrm{mV}$ was periodically applied to the membrane, and the membrane current steps recorded as a function of time on a plotter. To minimize electrode polarization, sintered Ag/AgC1 electrodes (type 140, Annex instruments, Santa Ana, CA) were used. A separate pair of electrodes was used for each type of experiment to avoid crosscontamination. The nonactin $-\mathrm{K}^{+}$and $\mathrm{TPhAs}^{+}$conductance was obtained from the steady state current measurements (using Model 135 electrometer, Princeton Applied Research, Princeton, NJ), while the TPhB ${ }^{-}$conductance was obtained by the voltage pulse method. The absence of diffusion polarization was checked in a separate experiment in which the time dependence of membrane current after the application of membrane bias 
was measured and the d.c. current magnitude monitored at various speeds of stirring bars and rotating magnets. The diffusion polarization due to unstirred layers was found to be insignificant. At high membrane currents only a small electrode polarization was observed (up to several $\mathrm{mV}$ ), for which the applied voltage was corrected.

The experimental current-voltage data have been analyzed according to the following procedure. First, the specific membrane conductance $G(V)$, was computed using the area of the hole $A$ as the surface area of the membrane. Second, the zero voltage conductance $G(0)$, was obtained from the best fit of the low voltage conductance data (typically up to $100 \mathrm{mV}$ ) to a polynomial of the second degree with respect to $V$. All kinetic information on the nonactin-mediated transport of $\mathrm{K}^{+}$was obtained from the voltage dependence of normalized membrane conductance $G(V) / G(0)$, and the zero voltage conductance $G(0)$. The membrane barrier distortion parameter $\omega$ has been set to 0.005 (Table 1 in Ref. 35) since the thickness of PC/cholesterol/n-decane membranes evaluated from the specific capacitance data of Hanai et al. (95) is about $3.2 \mathrm{~nm}$. The average number of membranes per one data point is seven; the error bar denotes " $n-1$ " standard deviation.

\section{4c. TRANSIENT CURRENT MEASUREMENTS AND ANALYSIS OF DATA}

The transient current measurements for $\mathrm{TPhB}^{-}$were performed using a two-electrode "voltage clamp" arrangement (Fig. 3). The electrodes used were sintered $\mathrm{Ag} / \mathrm{AgCl}$. A known voltage pulse was applied across the membrane by a pulse generator (Hewlett Packard, Loveland, C0). The membrane current signal was converted first into a voltage signal. The current-voltage converter, based on operational amplifier LH00602 


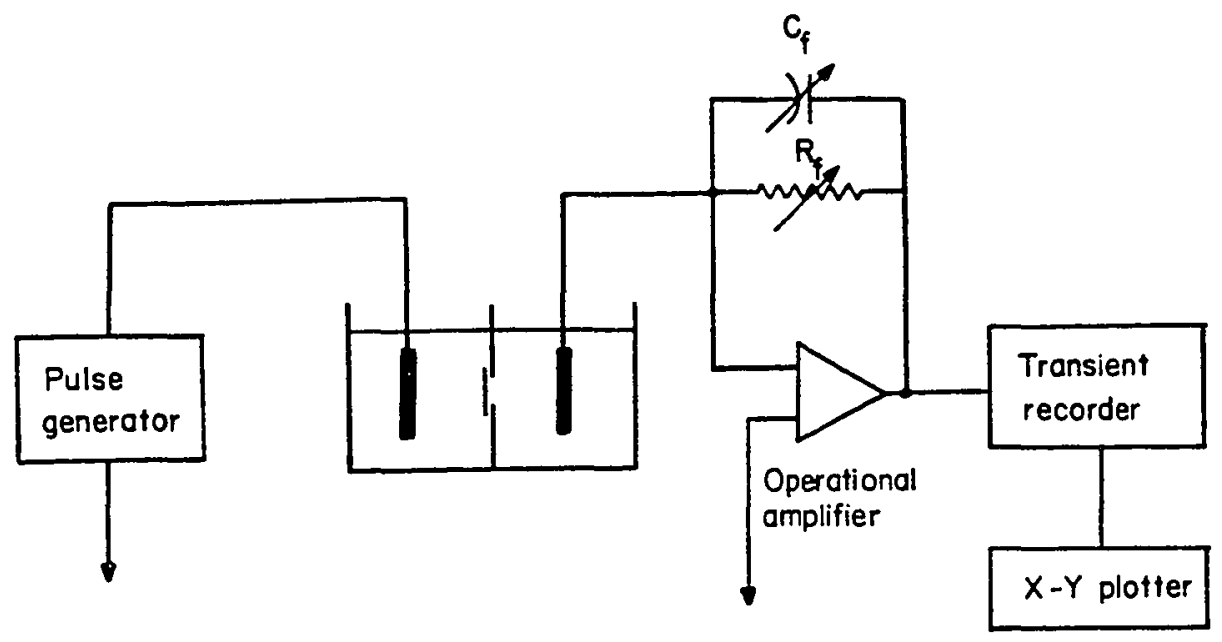

Figure 3. A block diagram of the experimental apparatus used for the transient current measurements.

(National semiconductor, Santa Clara, CA) was similar to that described by Sargent (96). The amplified transient current was first stored in a Biomation transient recorder (Model 802, Cupertino, CA), and then transferred to an $x-y$ plotter (Model 2000, Houston Instruments, Houston, TX). From the plot quantities of interest such as the initial membrane conductance $\left.G(V)\right|_{t=0}$ and the membrane current relaxation time constant $\tau(V)$ were obtained. From the fit of $I(V)=\left.I(V)\right|_{t=0}$. $\exp (-t / \tau(V))$ to the experimental data, the initial membrane current $\left.I(V)\right|_{t=0}$ and the relaxation time constant $\tau(V)$ were determined. The initial membrane conductance was calculated from the initial current density $\left.J(V)\right|_{t=0}=\left.I(V)\right|_{t=0} / A$, where $\left.I(V)\right|_{t=0}$ is the initial membrane conduction current and $A$ the area of the hole, and the applied potential difference $V,\left.G(V)\right|_{t=0}=\left.J(V)\right|_{t=0} / V$. The zero voltage conductance $G(0)$ and zero voltage time constant $\tau(0)$ were obtained by extrapolating low voltage data (up to $80 \mathrm{mV}$ in most cases) 
using a polynomial of the second degree. The amount of charge per unit membrane area $Q(V)$ transferred across the membrane during the transient conduction process was calculated according to Eq. 24 or 27. The total charge density due to $\mathrm{TPhB}^{-}$ions adsorbed at the membrane surface $Q_{\text {ads }}$ was assumed to be the average of $Q(V)$ measured at high membrane bias $(160,180,200$ and $220 \mathrm{mV}$ ) because tanh at these voltages approaches unity.

\section{4d. INTERFACIAL POTENTIAL MEASUREMENTS}

The method of measurement of interfacial potential difference was similar to that described in references 57 and 77 and was measured by means of a polonium electrode (Nuclear Products, E1 Monte, CA) and PAR electrometer, model 135. The aqueous solution on which the monolayer was spread was contained in a $100 \mathrm{~mm}$ crystallizing dish, and was electrically connected with a reference calomel electrode by means of a $\mathrm{KCl}$ bridge. The polonium electrode was placed several millimeters above the aqueous surface. In order to stabilize the potential of the measuring electrode, the electrode was kept for about half a day above the clean water surface before the start of the experiment. If the system was clean, the interfacial potential difference across the air/ deionized water (Millipore Q2 system, Millipore, Bedford, MA) surface was $-440 \pm 10 \mathrm{mV}$. The subphase aqueous solution contained $\mathrm{KCl}, 2,4-\mathrm{D}$, and buffer; LiCl was used for the adjustements of ionic strength. The experimental conditions are described in detail in the figure legends. The monolayer forming solution was prepared by dissolving lipids in hexadecane; $1 \%$ of methanol was also added to the lipid solution in order to improve lipid solubility. The cholesterol mole fraction was the same 
as in the membrane forming solution (i.e., 0.76). For PC-chol monolayers the monolayer forming solution contained $29 \mathrm{mg}$ of lecithin and cholesterol in one $\mathrm{ml}$ of hexadecane; for GMO monolayers the concentration of GMO in hexadecane was $30 \mathrm{mg} / \mathrm{ml}$.

The air/aqueous solution interfacial potential difference was recorded 15-20 minutes after the radioactive electrode was placed above the aqueous surface. About 3 microliters of lipid solution in hexadecane were then injected slowly along the surface of the crystallizing dish, and the new value of the potential difference was recorded after it stabilized. In each case, about 4 measurements for each monolayer have been made because of the surface potential fluctuations. It was also noticed that the surface potential of GMO monolayers fluctuated less than that of the PC-chol ones. After the measurement, the dish was rinsed thoroughly with methanol and deionized water, and the surface potential of clean water measured again in order to check the cleanliness of the equipment. Each data point represents an average for four monolayers and the error bar represents one standard deviation.

\section{4e. MATERIALS}

Chromatographically pure egg lecithin was prepared by Dr. Kwan Hsu (97), recrystallized cholesterol was a gift from Dr. McClure of the Chemistry Department, GMO was obtained from Applied Science Labs., Inc. (State College, PA), and $n$-decane, grade $99 \%+$, from Aldrich Chemical Co. (Milwaukee, WS). The tetraphenylarsonium chloride hydrate and sodium tetraphenylborate were obtained from Aldrich Chemical Co., 2,4-D and 2,4,5-T were gifts from Dow Corning Co. (Midland, MI) and 2,4-DB and 2,4-D-isobutyl ester were from Chapman Division of Rhodia 
Inc. (Portland, OR). Indole acetic acid was purchased from Sigma Chemical Co. (St. Louis, MO) and 2,4,6-T from Pfaltz and Bauer, Inc. (Stanford, Conn.). Leucine, isoleucine, and valine amino acid conjugates of 2,4-D were synthesized by Dr. A. S. Levinson of the Chemistry Department using the method of Wood and Fontain (98). Nonactin was a gift from the Squibb Institute of Medical Research (Princeton, N.J.). For the preparation of solutions, inorganic chemicals of analytical grade and deionized water from the millipore Q2 system (Millipore Corp., Bedford, Mass.) were used. 
CHAPTER V

EXPERIMENTAL RESULTS

5a. STUDIES OF THE EFFECT OF 2,4-D ON MEMBRANE TRANSPORT

\section{5a.1 2,4-D Induced Membrane Conductivity}

The current-voltage characteristics of lipid bilayer membranes were measured in the presence of 2,4-D (Fig. 4) in the aqueous solution. The $\mathrm{pK}_{\mathrm{a}}$ of 2,4-D is in the range of 2.6-2.8 $(99,100)$, therefore 2,4-D is mainly in the neutral form at $\mathrm{pH}$ lower than $\mathrm{pK}_{\mathrm{a}}$ and it is mainly in ionized form at $\mathrm{pH}$ higher than about $\mathrm{pH}$ 3. 2,4-D concentration in the aqueous solution was varied from zero to $1 \mathrm{mM}$ and $\mathrm{pH}$ from 1 to 6 . Under these conditions, 2,4-D did not increase the conductivity in lipid bilayer membranes.

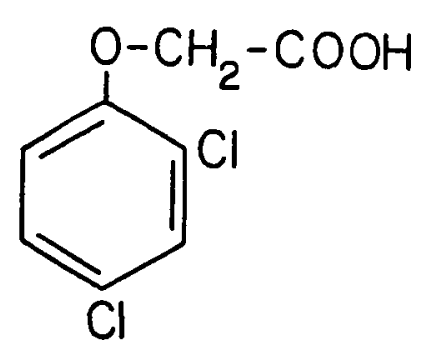

Figure 4. The structure of 2,4-D.

5a.2. Changes of Cationic and Anionic Membrane Conductance in the Presence of 2,4-D

To investigate the changes of cationic and anionic membrane conductance in the presence of 2,4-D, negatively charged tetraphenylborate 
$\left(\mathrm{TPhB}^{-}\right)$and positively charged ions, tetraphenylarsonium ( $\left.\mathrm{TPhAs}^{+}\right)$and nonactin-potassium complex (nonactin- $\mathrm{K}^{+}$) were used as probes. The induced conductance of PC-chol membranes due to these probe ions changed in the presence of 2,4-D. The experimental results are shown in Fig. 5, where the logarithm of zero voltage membrane conductance $G(0)$ is plotted versus the $\log$ of $2,4-D$ concentration. The results indicate that 2,4-D enhances transport of positive ions and, to a lesser degree, inhibits the transport of negative ions. The effect is very strong, the enhancement of nonactin- $\mathrm{K}^{+}$transport is as large as four orders of magnitude.

\section{5a.3. pH Effect}

The purpose of this experiment was to determine the relationship between the degree of conductivity enhancement on nonactin-mediated transport of potassium ions and the degree of ionization of 2,4-D. The 2,4-D enhanced conductance due to nonactin $-K^{+}$complex was measured as a function of $\mathrm{pH}$ and the results are shown in Fig. 6. As the results indicate, the enhancement is small at higher $\mathrm{pH}\left(\mathrm{pH}>\mathrm{pK}_{a} \simeq 2.6-2.8\right)$ in which 2,4-D is predominantly present in the anionic form. As the hydrogen ion concentration increases, the enhancement becomes more prominent, i.e., with increasing concentration of netural 2,4-D molecules. A similar study was done on membranes whose conductivity were induced by $\mathrm{TPhB}^{-}$. In this case the opposite effect was observed; at high $\mathrm{pH}$, the $\mathrm{TPhB}^{-}$conductance in the presence of 2,4-D is high, but it decreases as hydrogen ion concentration increases (data not shown). 


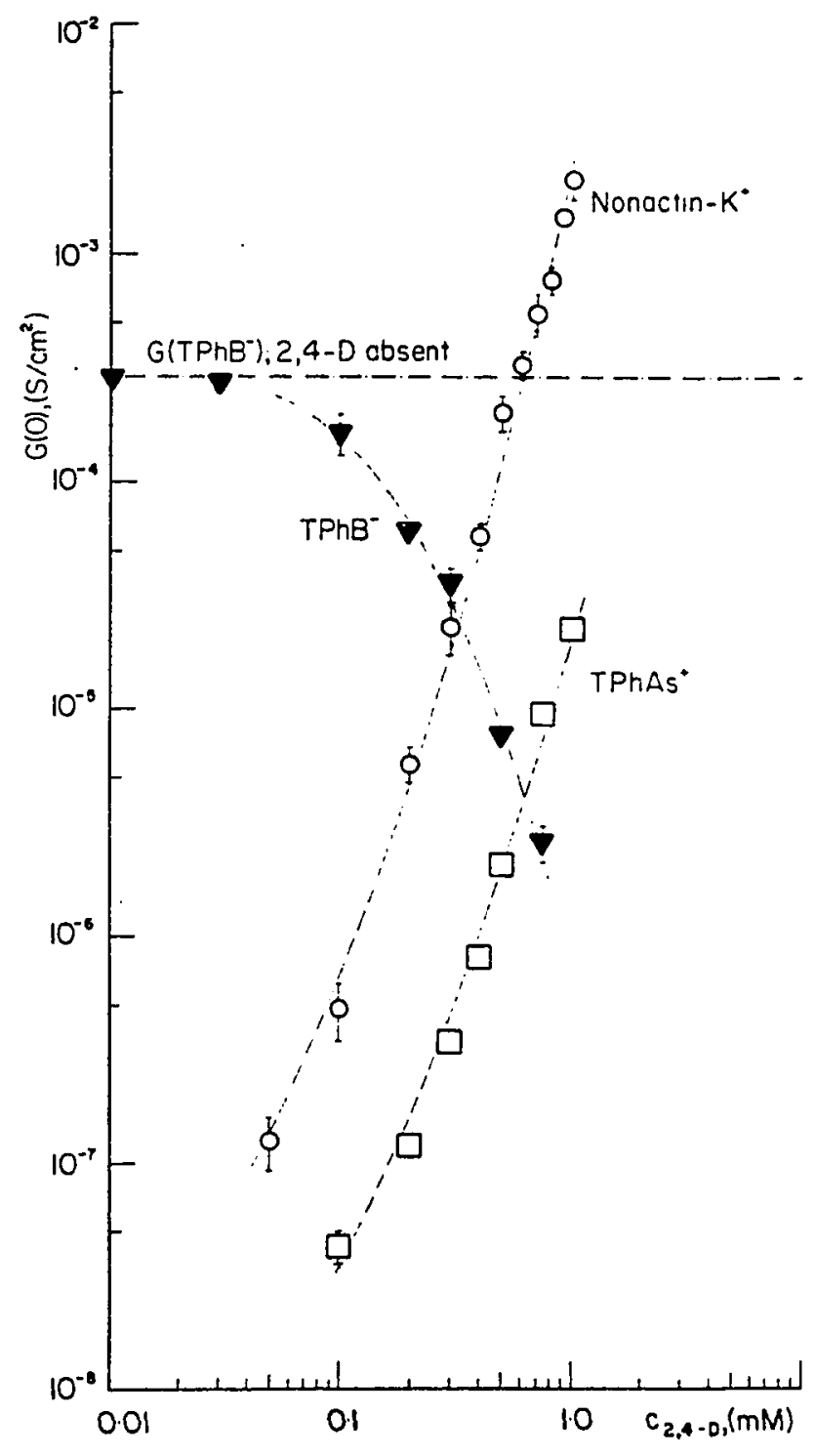

Figure 5. Effect of 2,4-D on the PC-chol membrane conductance due to nonactin, $\mathrm{TPhB}^{-}$and $\mathrm{TPhAs}^{+}$. Experimental conditions: $c_{\text {TPhAs }^{+}}=2 \times 10^{-3} \mathrm{M}$ in $0.5 \mathrm{M} \mathrm{LiCl} ; \mathrm{c}_{\mathrm{TPhB}^{-}}=1 \times 10^{-7} \mathrm{M}$ in $0.5 \mathrm{M} \mathrm{NaCl} ; c_{\text {nonactin }}$ (aqueous solution) $=1 \times 10^{-7} \mathrm{M}, c_{\text {nonactin }}$ (membrane solution) $=3 \times 10^{-5} \mathrm{M}, c_{\mathrm{K}^{+}}=0.06 \mathrm{M}$, ionic strength $(\mathrm{LiCl}+\mathrm{KCl})=1 \mathrm{M}, \mathrm{pH}=2$ (buffered in all experiments). 


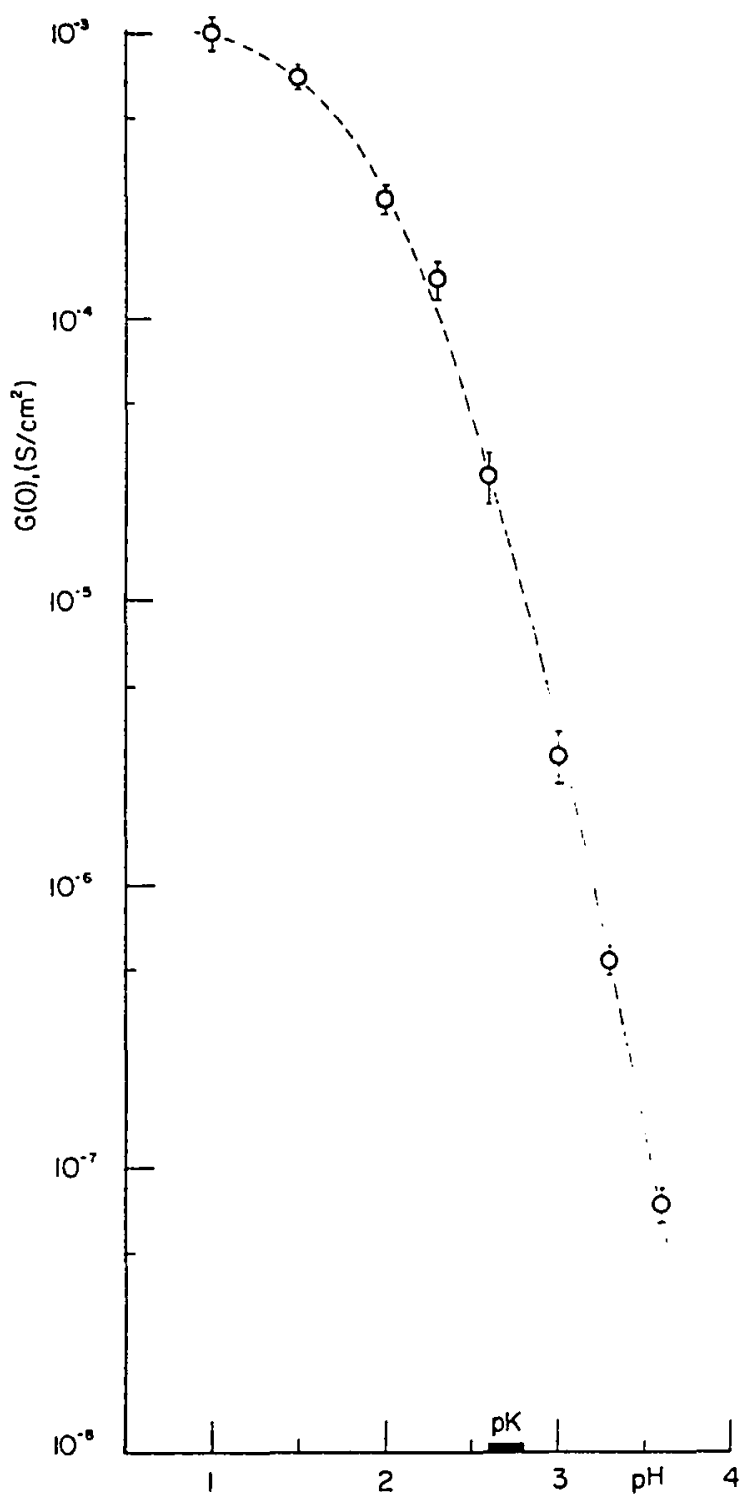

Figure 6. $\mathrm{pH}$ dependence of 2,4-D enhanced nonactin- $\mathrm{K}^{+}$conductance of PC-chol membranes. $c_{2,4-D}=5 \times 10^{-4} \mathrm{M}, c_{\text {nonactin }}$ (aqueous solution) $=3 \times 10^{-7} \mathrm{M}, c_{\text {nonactin }}$ (membrane solution) $=1 \times 10^{-4} \mathrm{M}$, $c_{\mathrm{K}^{+}}=0.06 \mathrm{M}$, ionic strength $(\mathrm{LiCl}+\mathrm{KCl})=1 \mathrm{M}$. 
A control experiment was performed to verify that the observed $\mathrm{pH}$ dependence is not due to experimental artifacts. As illustrated in Fig. 7 , within the experimental error, the nonactin $-\mathrm{K}^{+}$conductance is essentially independent of $\mathrm{pH}$ in the $\mathrm{pH}$ range between 1 and 10.5. This result suggests that the effect of expected surface charge at low $\mathrm{pH}$ due to ionized lecithin is insignificant, which is presumably due to high cholesterol content in the membrane.

In sum, the results indicate that the enhancement of cationic conductance, and the suppression of the anionic conductance are associated with the presence of neutral 2,4-D molecules, and that the negatively charged 2,4-D molecules are inactive.

5b. STUDIES OF THE 2,4-D ENHANCED TRANSPORT OF POSITIVE IONS

5b.1. Effect of 2,4-D on Voltage Dependence of Membrane Conductance Induced by Nonactin-Mediated Potassium Transport

The enhancement of nonactin-mediated transport of $\mathrm{K}^{+}$by 2,4-D was observed to be accompanied by the changes of voltage dependence of membrane conductance. These effects are illustrated in Figs. $8 a$ and $8 \mathrm{~b}$ where the dependence of zero voltage membrane conductance $G(0)$ on 2,4-D concentration, and the changes of the voltage dependence of the normalized membrane conductance $G(V) / G(0)$ are shown. With the increase of 2,4-D concentration, the membrane conductance $G(0)$ increases (Fig. 8a) and the nonactin- $\mathrm{K}^{+}$current-voltage curves changes from superlinear to sublinear (Fig. 8b). This suggests that the kinetics of nonactin-mediated potassium ion transport across the membrane changes in the presence of $2,4-D$. 


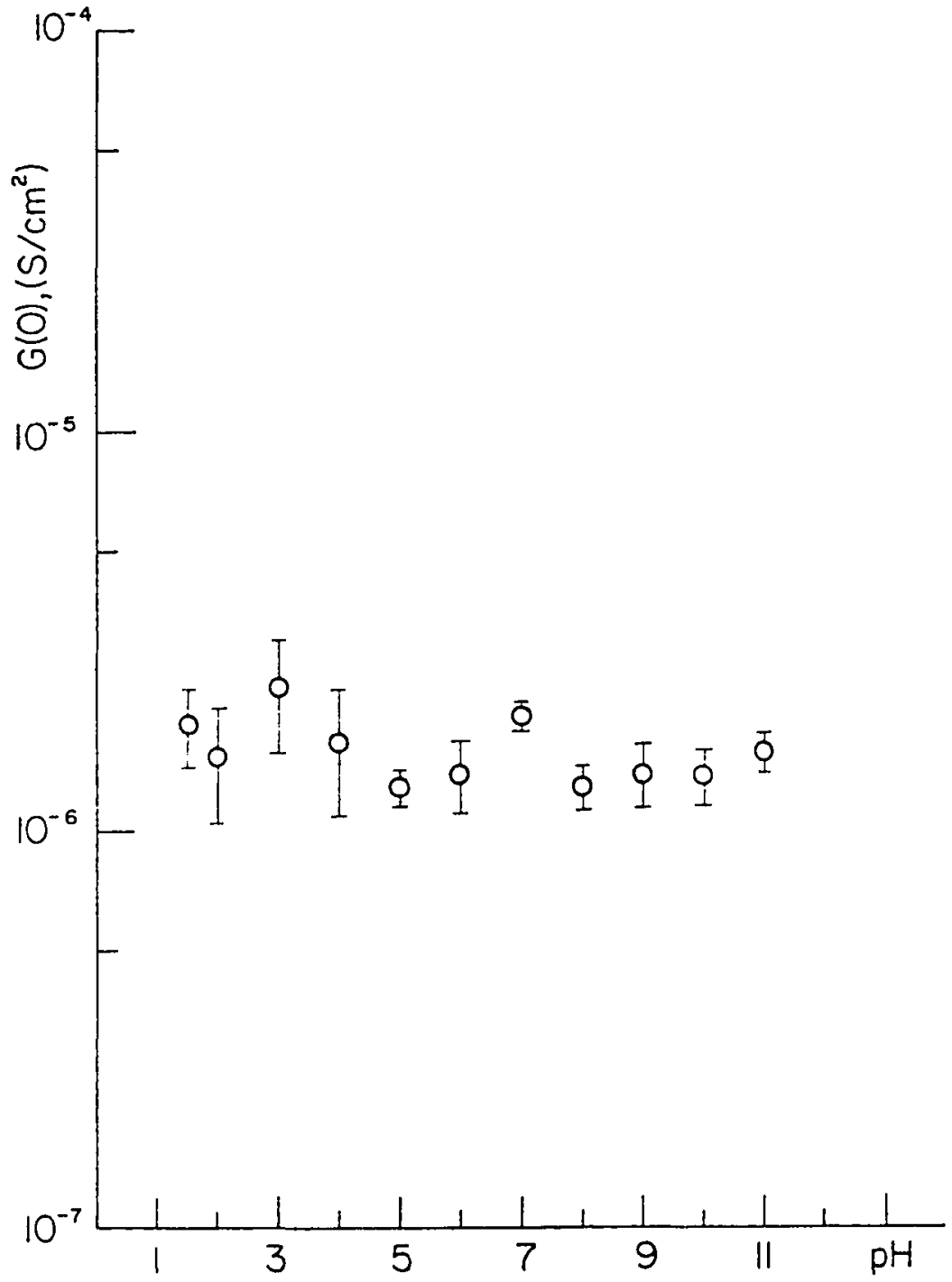

Figure 7. Control experiment. A demonstration that nonactin- $\mathrm{K}^{+}$ conductance of PC-chol membranes is independent of $\mathrm{pH}$ in the absence of 2,4-D. $c_{\text {nonactin }}$ (aqueous sol.) $=3.9 \times 10^{-6} \mathrm{M}, \mathrm{c}_{\mathrm{K}^{+}}=$ $0.2 \mathrm{M}$. 


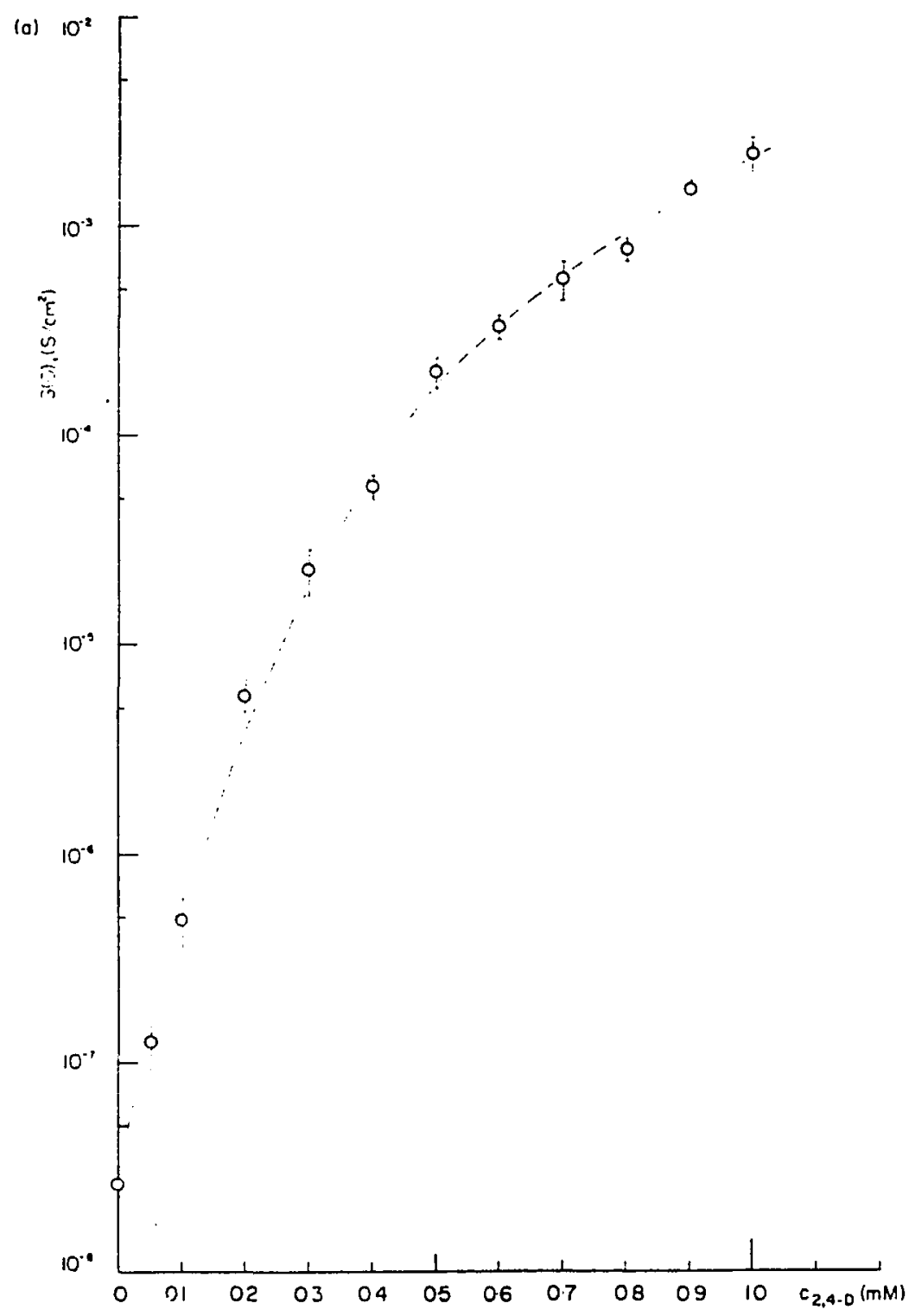

Figure 8a. 2,4-D enhancement of nonactin- $\mathrm{K}^{+}$conductance of PCchol membranes. Semilog plot of the 2,4-D concentration dependence of zero voltage conductance, Experimental conditions: $c_{\text {nonactin }}$ (aqueous solution) $=1 \times 10^{-7} \mathrm{M}$, $c_{\text {nonactin }}$ (membrane solution) $=3 \times 10^{-5} \mathrm{M}, \quad c_{\mathrm{K}^{+}}=0.06 \mathrm{M}$, ionic strength $(\mathrm{LiCl}+$ $\mathrm{KC} 1$ ) $=1 \mathrm{M}, \mathrm{pH}=2$ (buffered). 


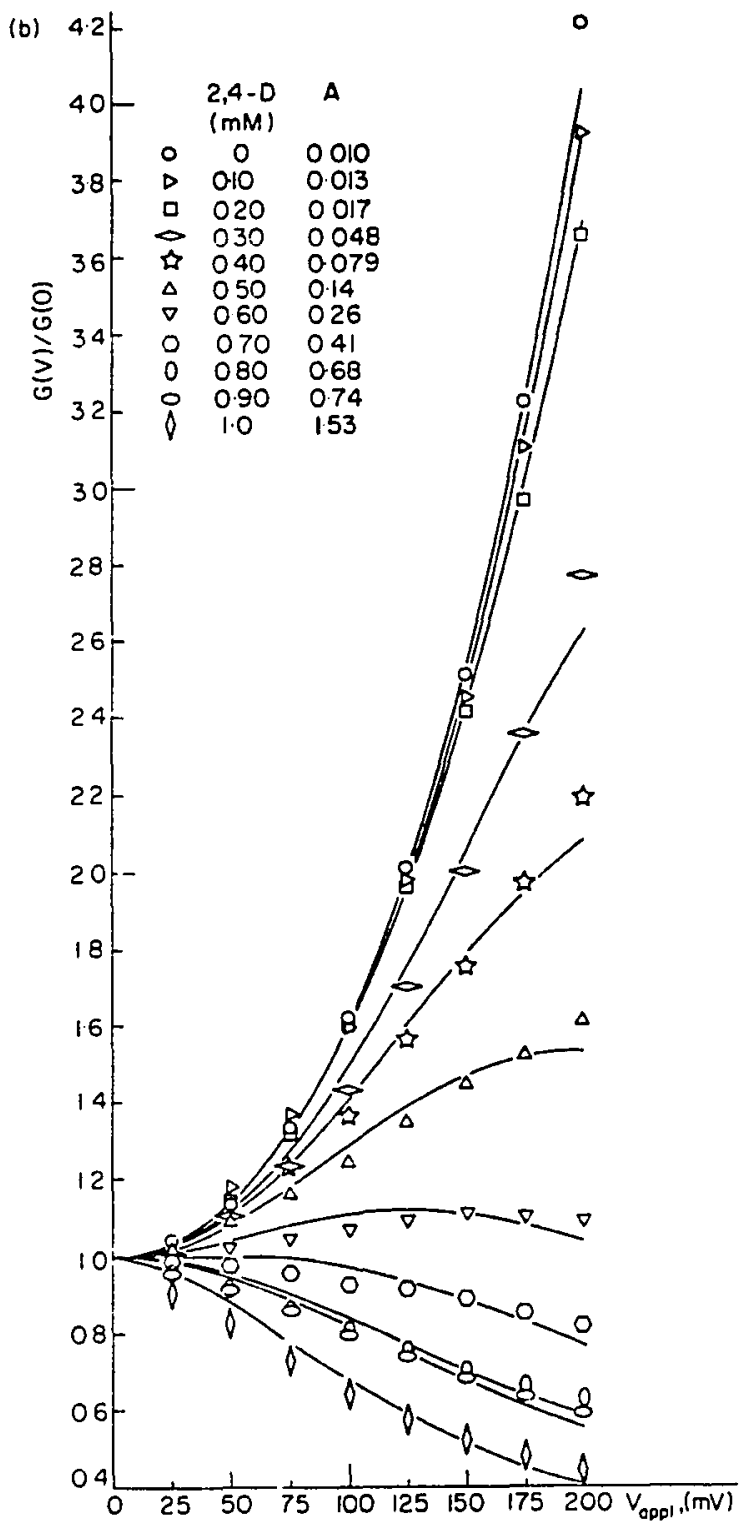

Figure 8b. Voltage dependence of 2,4-D enhanced conductance. Plot of normalized membrane conductance $G(V) / G(0)$ versus $V$ as a function of 2,4-D concentration. Each set of data points in this figure corresponds to one conductance point in Fig. 8a. The solid curves were obtained from Eq. 13 and represent the prediction of the transport model. Experimental conditions: $c_{\text {nonactin }}$ (aqueous solution) $=1 \times 10^{-7} \mathrm{M}$, $c_{\text {nonactin }}$ (membrane solution) $=3 \times 10^{-5} \mathrm{M}, \mathrm{c}_{\mathrm{K}^{+}}=0.06 \mathrm{M}$, ionic strength $(\mathrm{LiCl}=$ $\mathrm{KCl})=1 \mathrm{~m}, \mathrm{pH}=2$ (Buffered). 
5b.2. Dependence of Potassium Ion Concentration on the Conductance of Nonactin- $\mathrm{K}^{+}$Complex in the Presence of 2,4-D

The aim of this experiment was two-fold: (a) to investigate whether the binding constant of nonactin to potassium ion was affected by the presence of 2,4-D and, (b) to get a better insight into the observed kinetic limitations of the nonactin- ${ }^{+}$complex. Potassium ion concentration was varied at three different concentrations of 2,4-D: $0.35,0.5$ and $0.6 \mathrm{mM}$, while the total ionic strength was kept constant by adding appropriate amounts of LiCl (nonactin is an inefficient carrier of $\mathrm{Li}^{+}$ions). The membrane conductance as a function of potassium ion concentration is shown in Fig. 9. With exception of the case of the highest 2,4-D concentration and high $\mathrm{K}^{+}$concentration, the membrane conductance linearly increases with the concentration of $\mathrm{K}^{+}$ (Fig. 9a). Thus, the association reaction between $\mathrm{K}^{+}$and nonactin in the presence of 2,4-D remains to be of the first order. The results shown in Figs. 9b-d indicate that the upward curvature of $G(V) / G(0)$ vs $V$ decreases both with increasing potassium ion and 2,4-D concentration. Again, the changes of $d G / d V$ with the potassium ion concentration are observed only when 2,4-D is present; in the absence of 2,4-D the voltage dependence of conductance is always superlinear.

5c. STUDIES OF 2,4-D SUPPRESSED TRANSPORT OF NEGATIVELY CHARGED TETRAPHENYLBORATE: EFFECT ON TPhB- INDUCED MEMBRANE CONDUCTANCE, RELAXATION TIME CONSTANT AND NET TRANSFER OF CHARGE

Figures $10 \mathrm{a}$ and $10 \mathrm{~b}$ show the changes of the initial membrane conductance of $\mathrm{TPhB}^{-}$as a function of 2,4-D concentration for both switchON and switch-OFF conditions. The following two interesting properties 


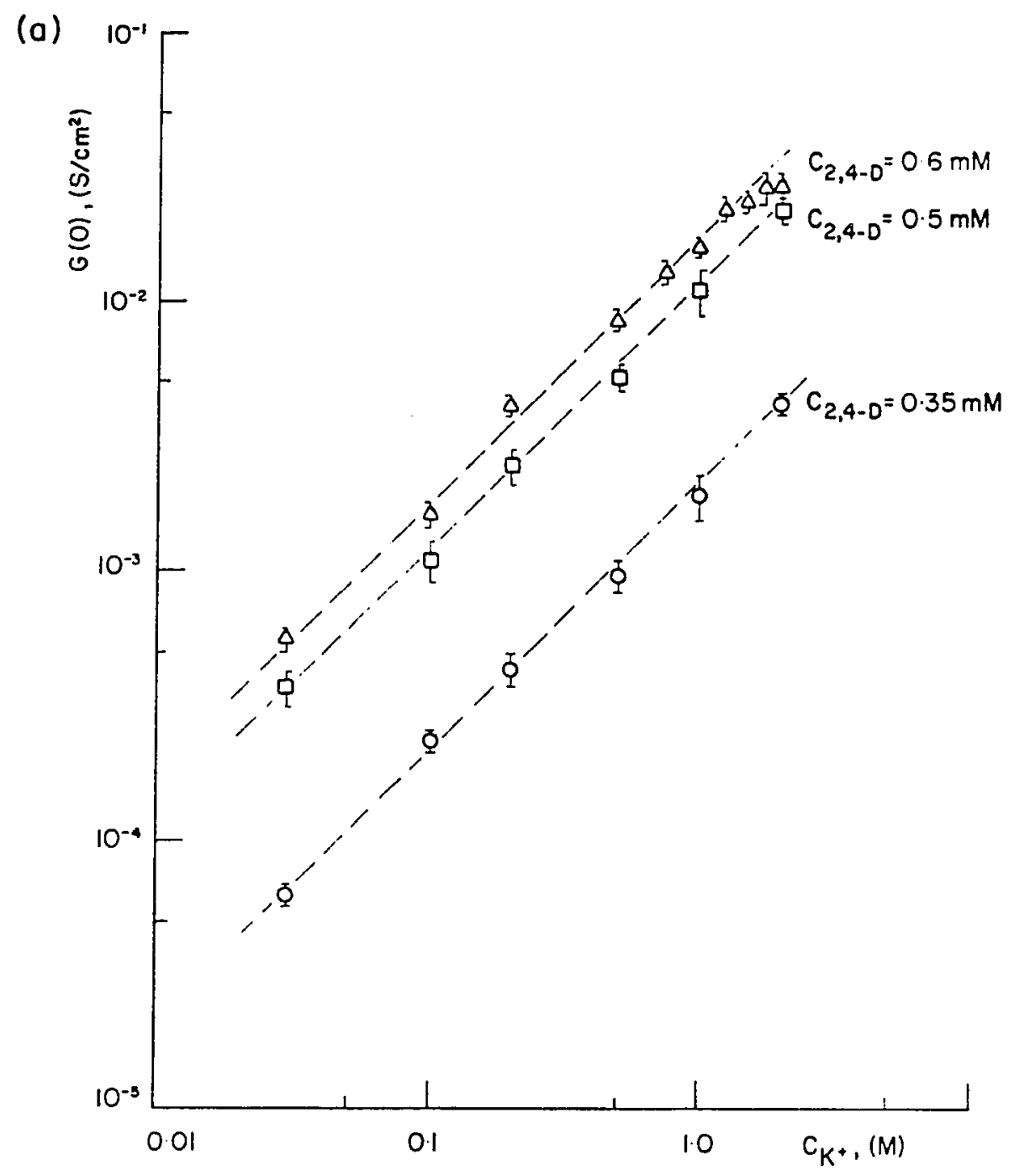

Figure 9a. Dependence of 2,4-D enhanced nonactin- $\mathrm{K}^{+}$conductance on potassium ion concentration. C nonactin (aqueous solution) = $4.5 \times 10^{-8} \mathrm{M}, \mathrm{c}_{\text {nonactin }}$ (membrane solution) $=1.4 \times 10^{-5} \mathrm{M}, \mathrm{pH}=2$ (buffered), ionic strength $(\mathrm{LiCl}+\mathrm{KCl})=2 \mathrm{M}$. The broken 1 ines are straight lines with slope of 1. 

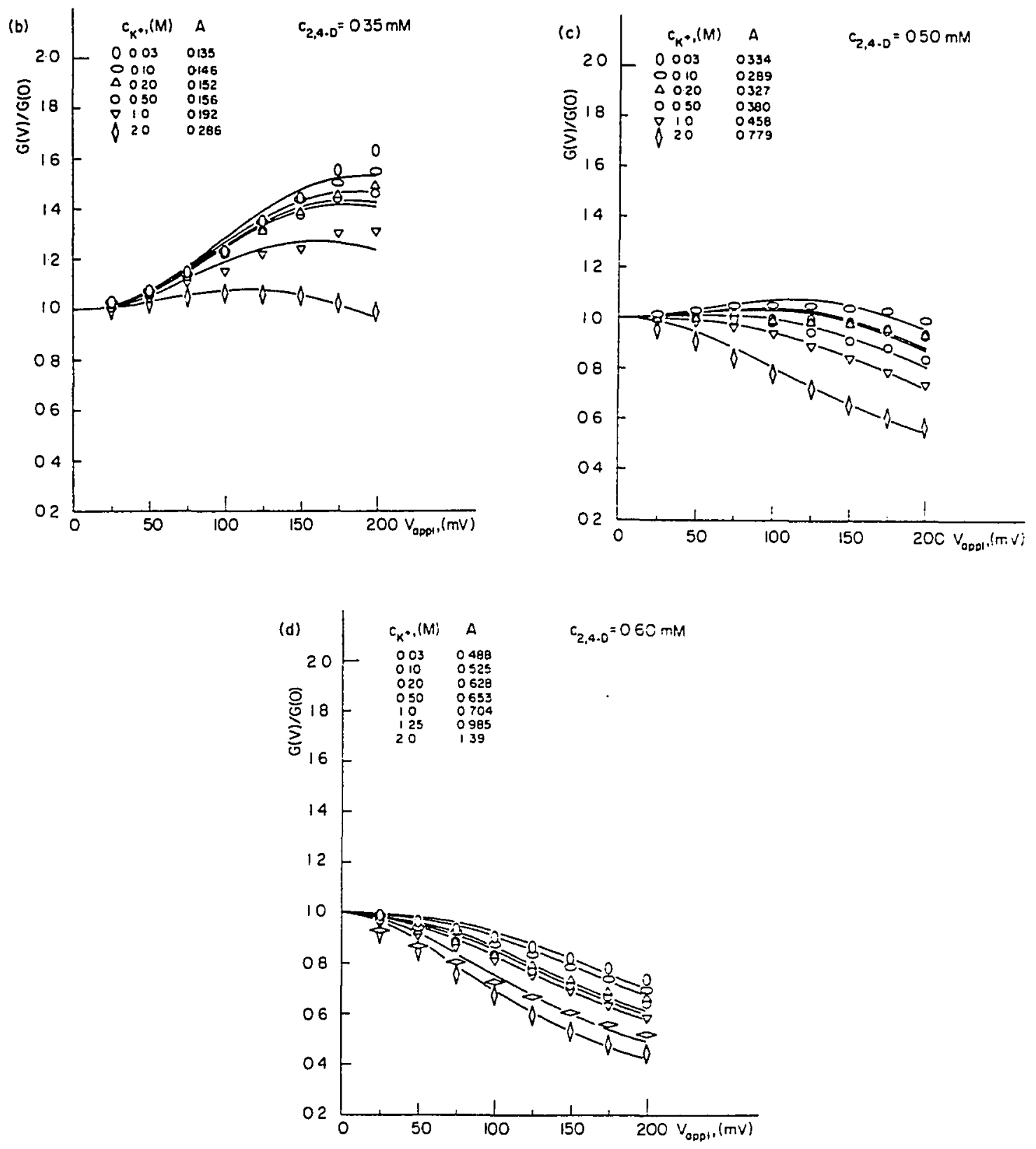

Figure 9b-d. Effect of potassium ion concentration on voltage dependence of normalized membrane conductance at three concentrations of 2,4-D. The solid curves were obtained according to Eq. 13 and represent the prediction of the nonactin- $\mathrm{K}^{+}$transport model. $c_{\text {nonactin }}$ (aqueous solution) $=4.5 \times 10^{-8} \mathrm{M}, c_{\text {nonactin }}$ (membrane solution) $=1.4 \times 10^{-5} \mathrm{M}, \mathrm{pH}=2$ (buffered), ionic strength (LiCl+ $\mathrm{KCl})=2 \mathrm{M}$. (b) $\mathrm{c}_{2,4-\mathrm{D}}=0.35 \mathrm{mM}$; (c) $\mathrm{c}_{2,4-\mathrm{D}}=0.5 \mathrm{mM}$;

(d) $c_{2,4-D}=0.6 \mathrm{mM}$. 


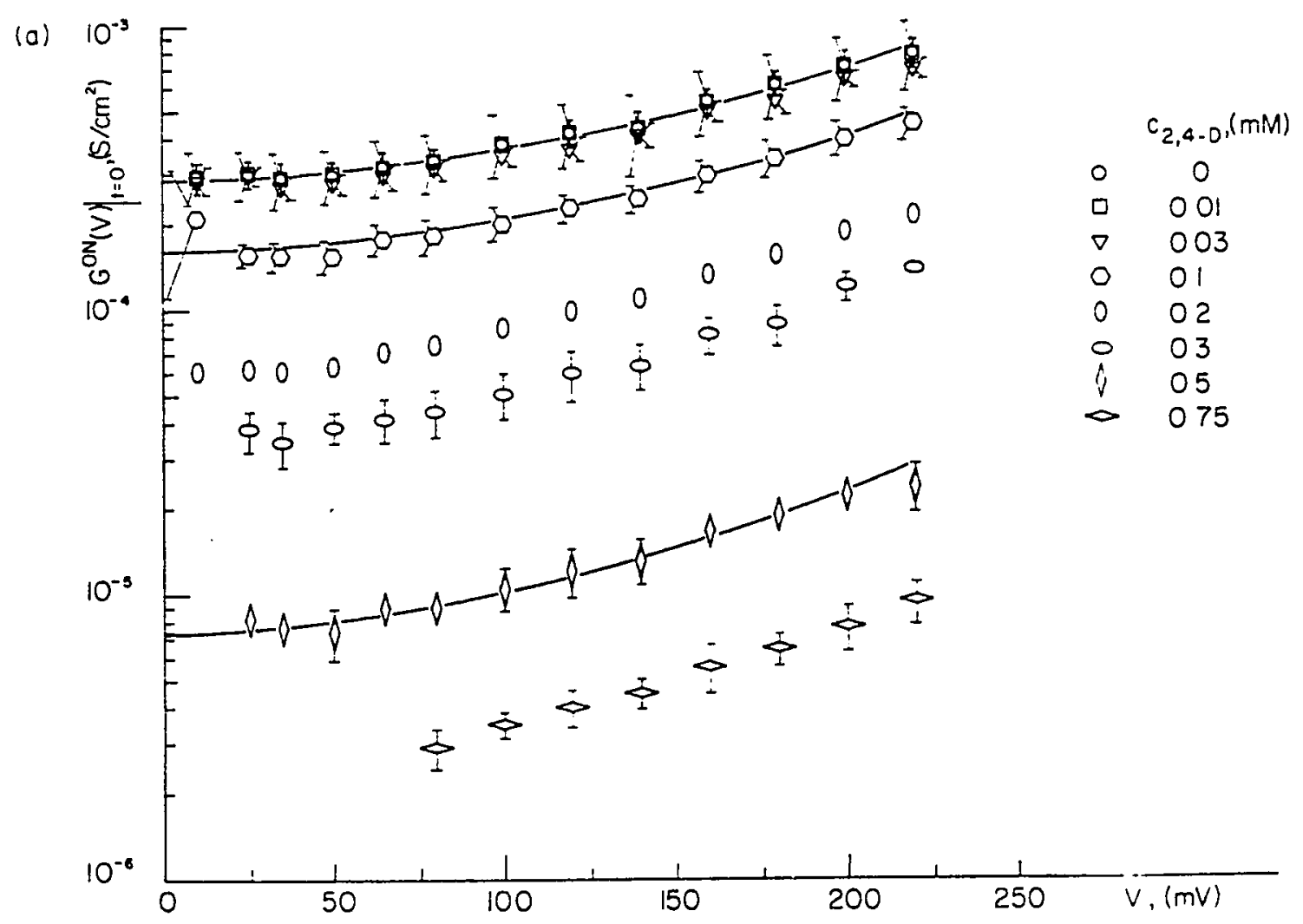

Figure 10a. Voltage dependence of initial $\mathrm{TPhB}^{-}$conductance of

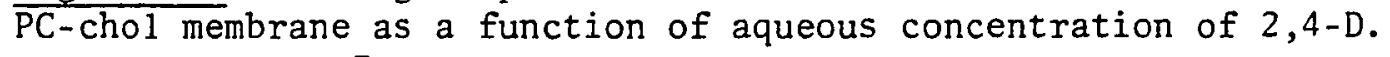
$c_{\mathrm{TPhB}^{-}}=1 \times 10^{-7} \mathrm{~m}, \mathrm{pH}=2$ (buffered), $c_{\mathrm{NaCl}}=0.5 \mathrm{M}$. Conductance determined from the transient current observed after the application of membrane bias (switch-ON condition). The solid curves represent the fit of Eq. 20 to the data for 2,4-D concentrations $0,1 \times 10^{-4} \mathrm{M}$, and $5 \times 10^{-4} \mathrm{M}$. 


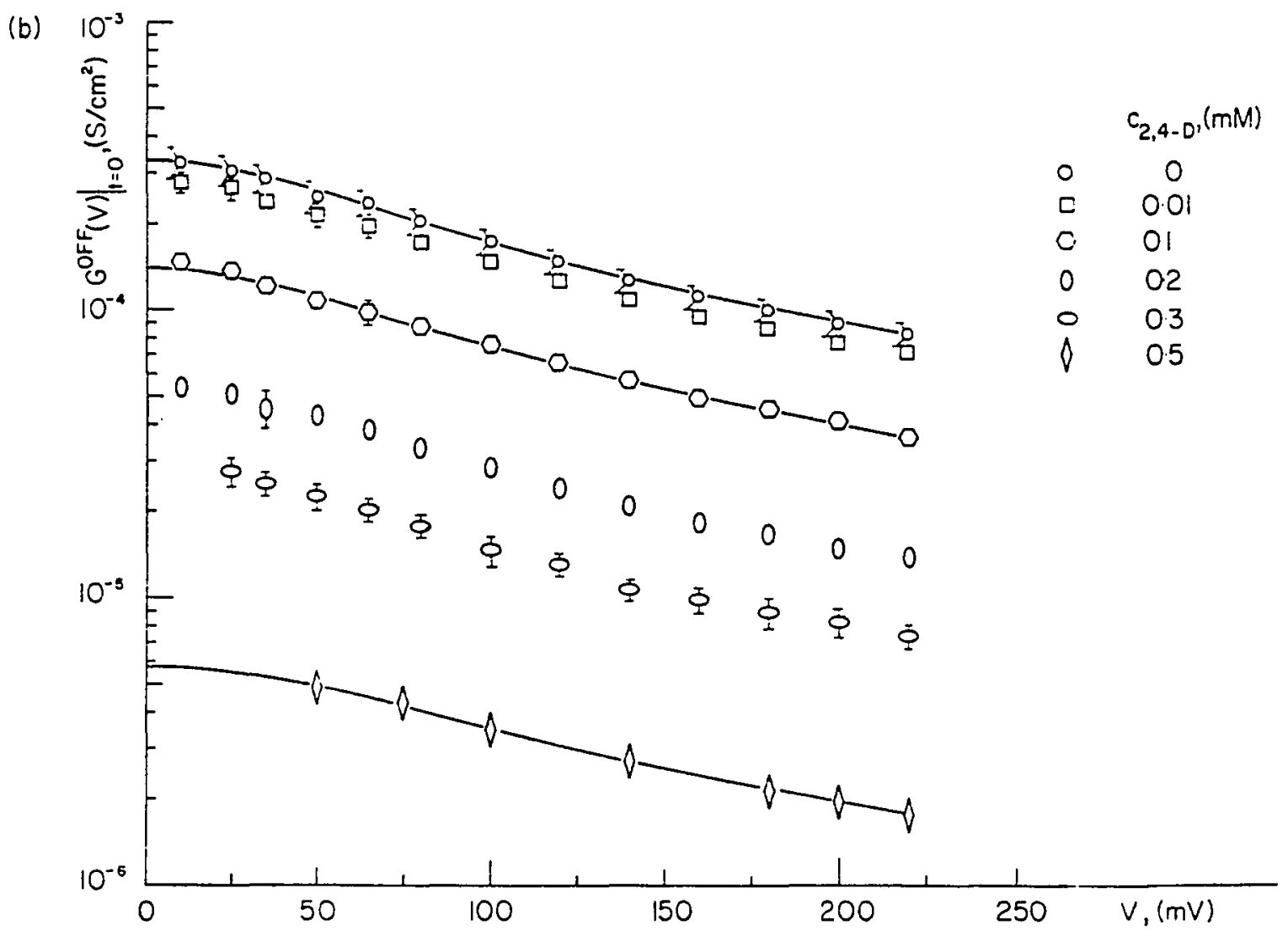

Figure 10b. Voltage dependence of initial $\mathrm{TPhB}^{-}$conductance of PC-chol membrane as a function of aqueous concentration of 2,4-D. $c_{\mathrm{TPhB}^{-}}=1 \times 10^{-7} \mathrm{M}, \mathrm{pH}=2$ (buffered), $c_{\mathrm{NaCl}}=0.5 \mathrm{M}$. Conductance determined from the transient current observed after the removal of membrane bias (switch-OFF condition). The solid curves represent the fit of Eq. 25 to the data for 2,4-D concentrations $0,1 \times 10^{-4} \mathrm{M}$, and $5 \times 10^{-4} \mathrm{M}$. 
were observed. First, the magnitude of the initial membrane conductance decreased with increasing concentration of 2,4-D and second, the voltage dependence of conductance with 2,4-D was essentially unchanged. Figures 11a and 11b show the changes in relaxation time constant in the presence of 2,4-D. The suppression of the conductance by 2,4-D is associated with the increase of the relaxation time constant (Fig. 11), which is the measure of ion redistribution time in the membrane interior. For a $2,4-\mathrm{D}$ concentration change from 0 to $7.5 \times 10^{-4} \mathrm{M}$, the membrane conductance of PC-chol membranes decreased by a factor of about 100, and the relaxation time constant increased by about 30 fold. The solid curves in Figs. 10 and 11 represent the least square fit of Eqs. $20,22,25,26$ to the experimental results. The net charge per unit membrane area, translocated across the membrane as a function of applied potential difference and 2,4-D concentration, is given in Fig. 12. At high voltages, the net transferred charge becomes constant, and this limiting value of $Q(V)$ is equal to the surface density due to adsorbed $\mathrm{TPhB}^{-}$ions. $\mathrm{Q}_{\mathrm{ads}}$. The results indicate that 2,4-D inhibits adsorption of $\mathrm{TPhB}^{-}$since $Q_{\text {ads }}$ decreases with increasing 2,4-D concentration.

\section{5d. IONIC STRENGTH DEPENDENCE OF MEMBRANE CONDUCTANCE}

It was found that in the presence of $2,4-D$, nonactin-mediated transport of $\mathrm{K}^{+}$becomes sensitive to ionic strength of the aqueous solution. Aqueous 2,4-D concentration in this experiment was kept at $7.5 \times 10^{-4} \mathrm{M}$ and the ionic strength has been changed from 0.1 to $2 \mathrm{M}$. Principal features of the ionic strength effect are depicted in Fig. 13. With the increase of ionic strength the membrane conductance in- 
(c)

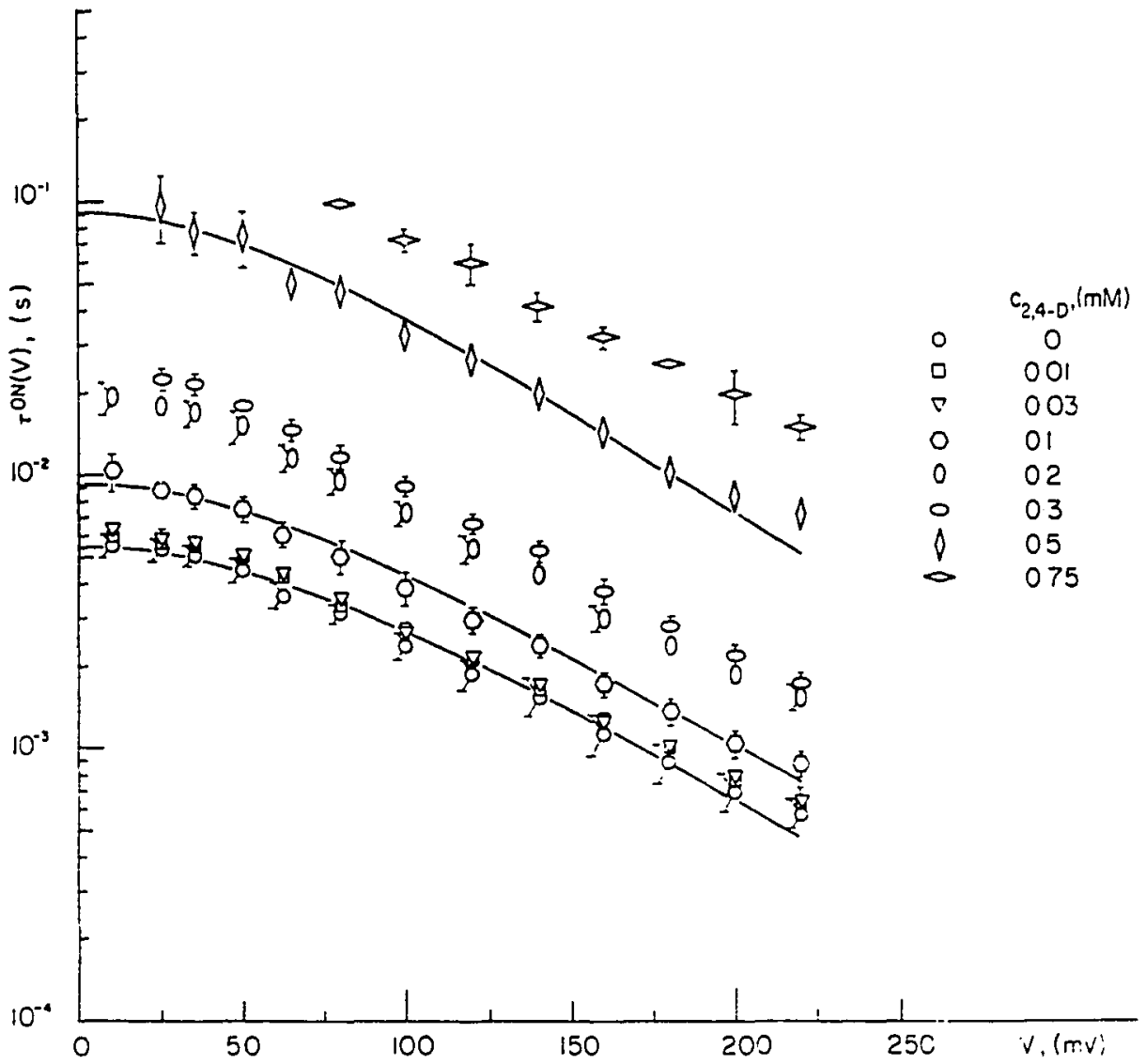

Figure 1la. Voltage dependence of membrane current relaxation time constant as a function of aqueous 2,4-D concentration. Experimental conditions are identical to those given in Fig. 10. The time constant determined from the transient current observed after the application of bias voltage across the membrane (switchON condition). The solid curves represent fit of Eq. 22 to the data for 2,4-D concentrations $0,1 \times 10^{-4} \mathrm{M}$, and $5 \times 10^{-4} \mathrm{M}$. 


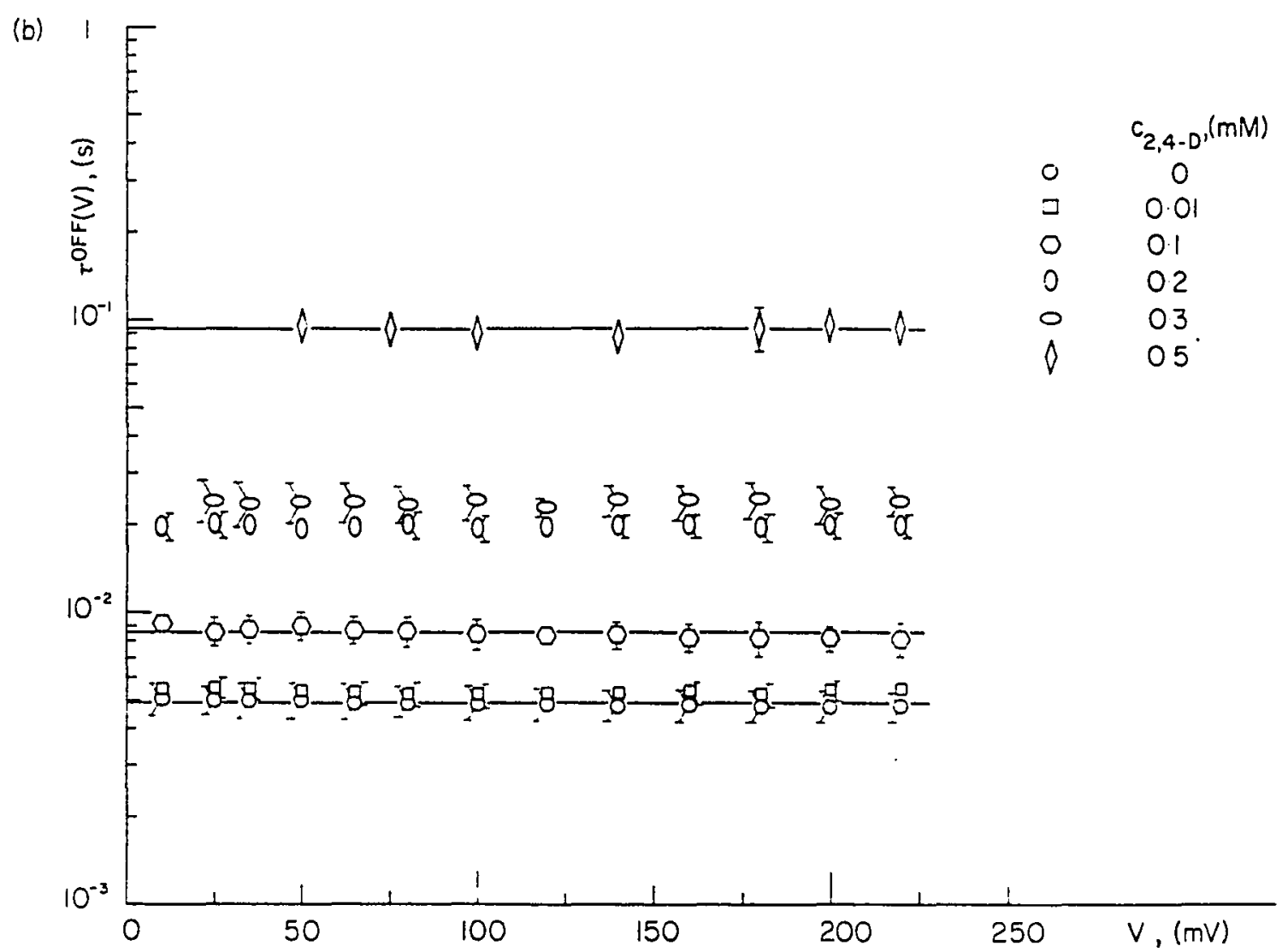

Figure 11b. Voltage dependence of membrane current relaxation time constant as a function of aqueous 2,4-D concentration. Experimental conditions are identical to those given in Fig. 10. The time constant determined from the transient current observed after the removal of bias voltage (switch-OFF conditions). The straight lines represent the average time constant. For the purpose of illustration, 2,4-D concentrations $0,1 \times 10^{-4}$, and $5 \times 10^{-4}$ have been chosen. 


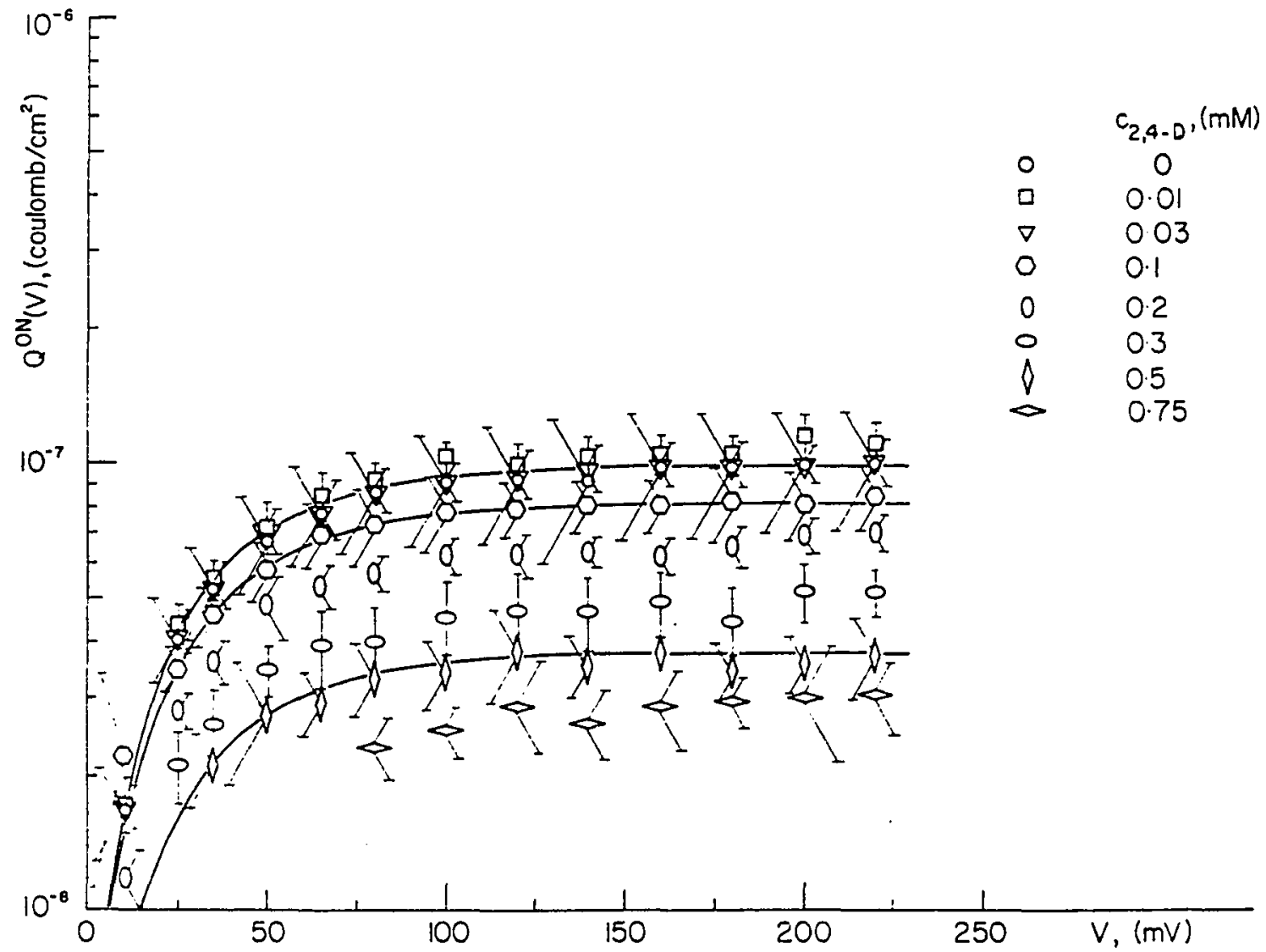

Figure 12. The effect of 2,4-D on the transfer of electric charge associated with $\mathrm{TPhB}^{-}$ions across $\mathrm{PC}$-chol membranes. The solid curves have been drawn according to Eq. 24 for $\beta=0.91$. 
creases (Fig. 13a), and the first derivative of the conductance with respect to applied voltage, $\mathrm{dG} / \mathrm{dV}$, monotonically decreases (Fig. 13b). In the control experiment, i.e., dependence of ionic strength on the nonactin- $\mathrm{K}^{+}$induced conductivity in the absence of $2,4-\mathrm{D}$, (a) the zero voltage conductance $\mathrm{G}(0)$, and (b) the normalized membrane conductivity $G(V) / G(0)$ are both independent of the ionic strength (Figs. 13a and 13c). This result is in agreement with findings of other workers $(81,88)$ obtained under different conditions.

The effect of ionic strength in the presence of 2,4-D appears to be a general one. Both the TPhAs ${ }^{+}$and the nonactin- ${ }^{+}$conductance increase with increasing ionic strength in the presence of $2,4-D$, and the conductance changes are very similar (Fig. 13a). Since 2,4-D suppresses the $\mathrm{TPhB}^{-}$conductance, an opposite dependence on ionic strength was expected in the case of $\mathrm{TPhB}^{-}$. This possibility was tested at two concentration levels of $\mathrm{LiCl}: 0.1$ and $2 \mathrm{M}$, and 2,4-D concentration of $7.5 \times 10^{-4} \mathrm{M}$. Under these conditions, the conductance change was found to be insignificant. Although the conductance effect was absent, an increase of the density of adsorbed $\mathrm{TPhB}^{-}(2.1$ fold $)$ and an increase of the relaxation time constant (2.2 fold) were observed at higher ionic strength. Those two effects cancel each other. In contrast, in the absence of 2,4-D the $\mathrm{TPhB}^{-}$membrane conductance increased with ionic strength ( 1.7 fold). This change is associated with the increased adsorption of $\mathrm{TPhB}^{-}$(2.5 fold) and a small increase (1.2 fold) in the relaxation time constant. The latter is a consequence of greater adsorption of $\mathrm{TPhB}^{-}(40)$. 


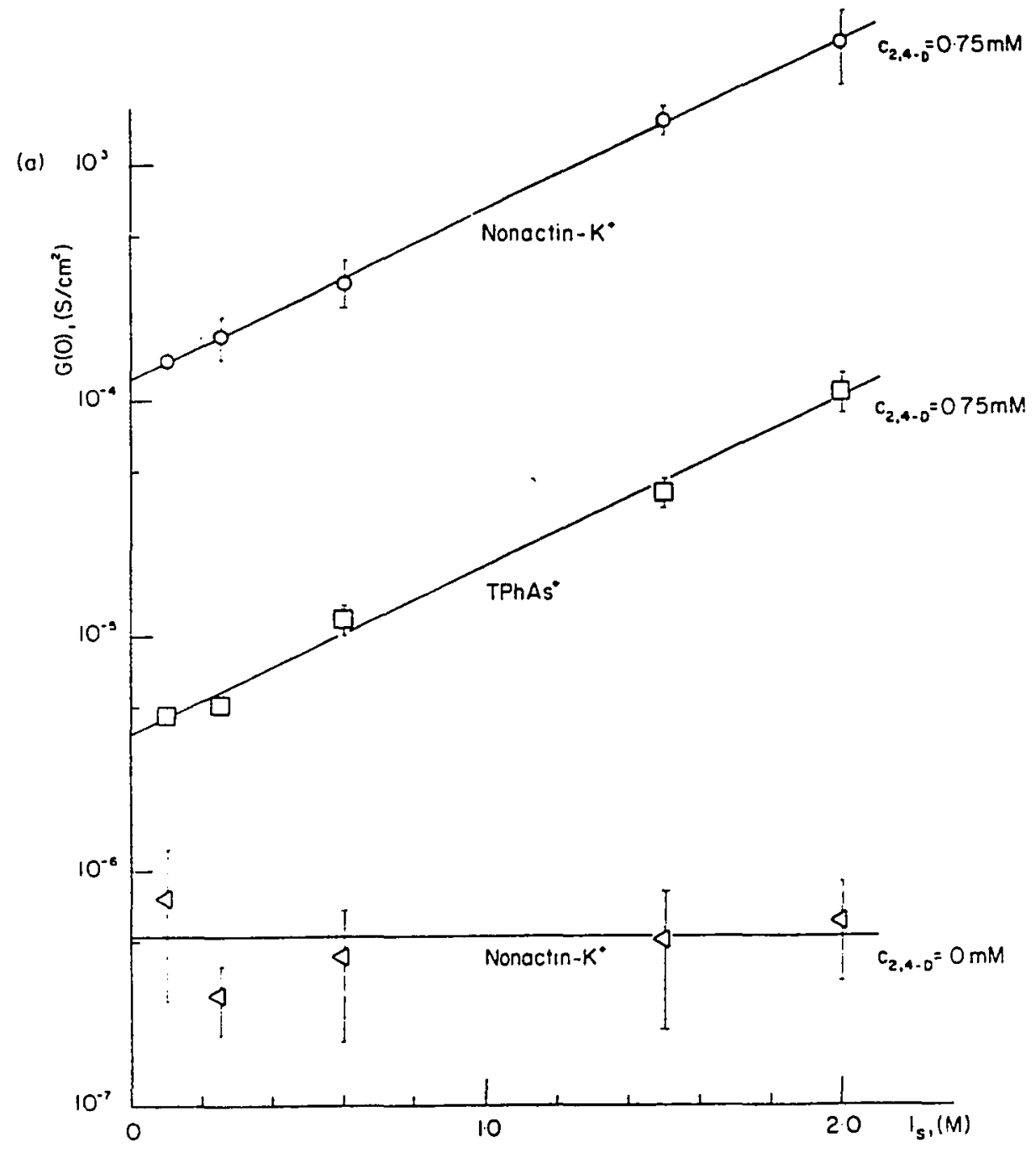

Figure 13a. Effect of ionic strength on the magnitude of membrane conductance associated with the transport of nonactin- $\mathrm{K}^{+}$and $\mathrm{TPhAs}^{+}$ ions across the PC-chol membranes. $c_{2,4-D}=7.5 \times 10^{-4} \mathrm{M}$, $c_{\text {nonactin }}$ (aqueous solution) $=4.5 \times 10^{-8} \mathrm{M}, c_{\mathrm{K}^{+}}=0.1 \mathrm{M}, \mathrm{c}_{\mathrm{TPhAs}^{+}}=$ $2 \times 10^{-3} \mathrm{M}, \mathrm{pH}=2$ (buffered). Control experiment: $c_{2,4-\mathrm{D}}=0$, $c_{\text {nonactin }}$ (aqueous solution) $=3.5 \times 10^{-6} \mathrm{M}$, $c_{\text {nonactin }}$ (membrane solution) $=1.9 \times 10^{-4} \mathrm{M}, c_{K^{+}}=0.1 \mathrm{M}, \mathrm{pH}=2$ (buffered). Top two solid lines represent the linear least square fit of $10 \mathrm{~g}$ $G(0)$ vs ionic strength. The bottom straight line represents the average of zero voltage nonactin- $\mathrm{K}^{+}$conductance. 


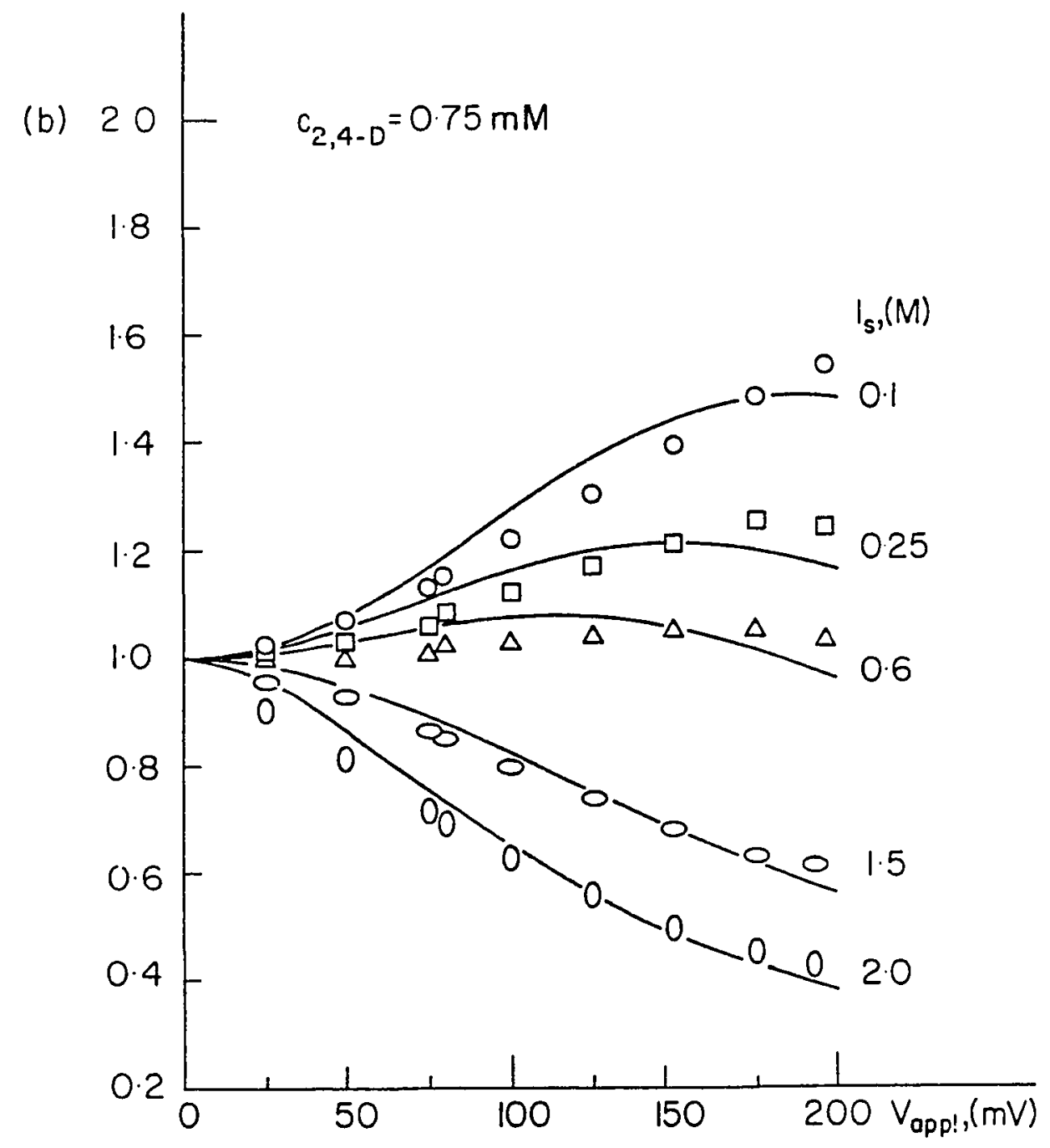

Figure 13b. Effect of ionic strength on voltage dependence of normalized membrane conductance associated with transport of nonactin- $\mathrm{K}^{+}$complex. Each set of data points corresponds to one conductance point in Fig. 13a. The solid curves were obtained from Eq. 13 and represent the prediction of the transport model. $c_{2,4-D}=7.5 \times 10^{-4} \mathrm{M}, c_{\text {nonactin }}$ (aqueous solution) $=4.5 \times 10^{-8} \mathrm{M}$, $c_{\mathrm{K}^{+}}=0.1 \mathrm{M}, \mathrm{pH}=2$ (buffered). 


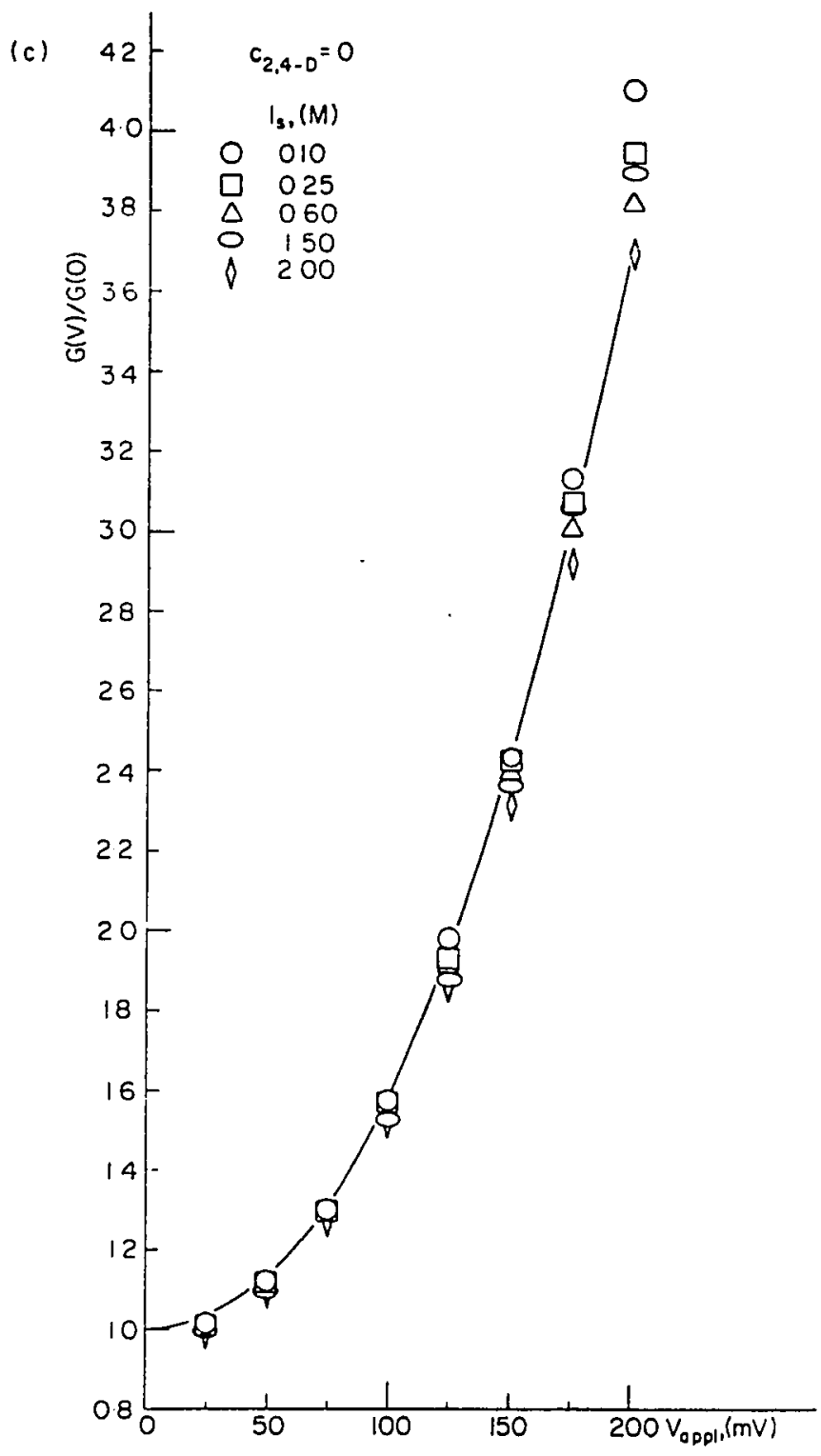

Figure 13c. Control experiment. A demonstration that in the absence of 2,4-D, the effect of ionic strength on the voltage dependence of nonactin- $\mathrm{K}^{+}$conductance is absent. $c_{2,4-\mathrm{D}}=0$, $c_{\text {nonactin }}$ (aqueous solution) $=3.5 \times 10^{-6} \mathrm{M}, \mathrm{c}_{\text {nonactin }}$ (membrane solution) $=1.9 \times 10^{-4} \mathrm{M}, \mathrm{c}_{\mathrm{K}^{+}}=0.1 \mathrm{M}, \mathrm{pH}=2$ (buffered). The solid curve was obtained from Eq. 13 and represents the prediction of nonactin- $\mathrm{K}^{+}$transport model. 
5e. RELATIONSHIP BETWEEN THE MEMBRANE STRUCTURE AND THE EFFECT OF 2,4-D INDUCED MODIFICATION OF MEMBRANE ION TRANSPORT

This experiment was designed to investigate whether the observed effect of 2,4-D on ion transport in PC-chol membranes is associated with the exclusion of cholesterol from the membrane into the membrane torus. In this case cholesterol free glycerolmonooleate (GMO) memebranes were used. If the presence of cholesterol in the membrane was critical, the conductance effect of 2,4-D would be absent. In contrast to this expectation, the conductance characteristics of GMO membranes changed in a manner similar to those found for PC-chol membranes (Sec. 5b.1, Fig. 8). The membrane conductance $G(0)$ increased and simultaneously the slope of $d G / d V$ decreased in the presence of 2,4-D (data not shown). The result suggest the effect of 2,4-D is not lipid specific.

5f. ELECTRICAL POTENTIAL DIFFERENCE ACROSS AIR/WATER AND AIR/LIPID MONOLAYER/WATER INTERFACES

The measurements of surface potentials were done under conditions similar to those for which a modified electrical conductivity of membranes was observed. The same electrolyte solution was used as the subphase and the same lipid composition of solutions were used for monolayers as for bilayer membranes. Even in the absence of 1ipid monolayer, 2,4-D changes the electrical potential difference across the air/water interface. Therefore, the experimental results for both types of interfaces are presented separately (Fig. 14). The data represent the electric potential difference between the ionizing electrode (air) and the reference electrode which was in contact with 


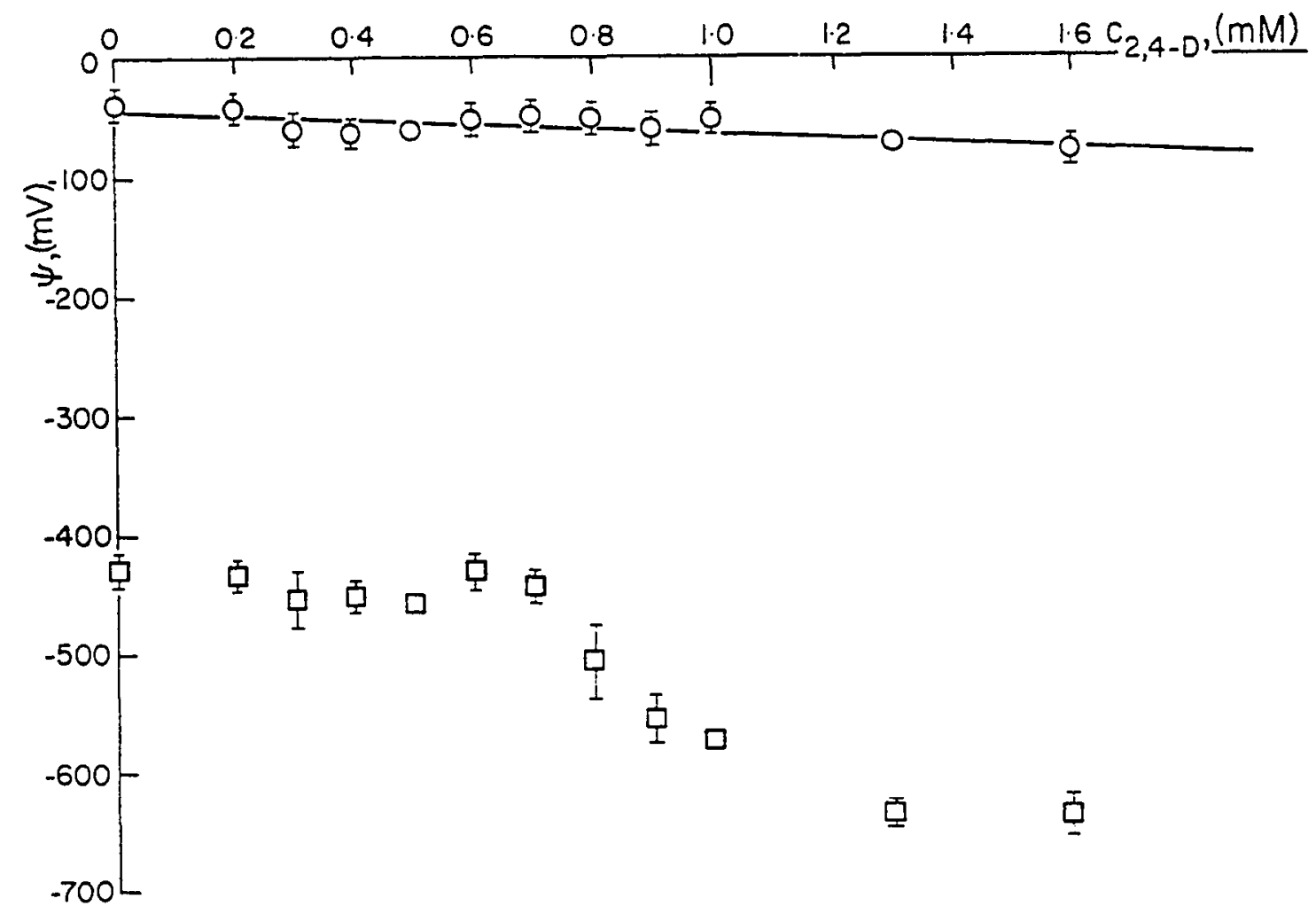

Figure 14. Changes of interfacial potential difference across the PC-chol monolayer (top) and the air/aqueous solution interface (bottom) as a function of aqueous concentration of 2,4-D. $\mathrm{pH}=2$ (buffered), $\mathrm{c}_{\mathrm{KCl}}=0.5 \mathrm{M}$. The solid line represent the least square fit of $\Delta \psi=b c_{2,4-D^{\circ}}$ 
the subphase (aqueous solution). In the absence of 2,4-D, the monolayer potential difference defined as $\psi_{\mathrm{PC}-\mathrm{chol}}=\Delta \mathrm{V}$ (air/PC-chol monolayer) water) - $\Delta V$ (air/water), is $406 \pm 7 \mathrm{mV}$, which agrees closely with $420 \mathrm{mV}$ reported for PC-chol monolayers by Hladky and Haydon (58) and $400 \mathrm{mV}$ by Anderson et al. (35), and $425 \mathrm{mV}$ for BPE monolayers (35). With increasing 2,4-D concentration, the potential difference across the air/water interface, i.e., the potential of the air side of the interface becomes more negative. A similar effect, but to a lesser degree, is observed in the PC-chol monolayers. ( $\mathrm{pH}$ in this experiment was kept at 2). Since the decrease of the surface potential can be also due to the presence of 2,4-D anions at the interface, the dependence of interfacial potential difference on $\mathrm{pH}$ was also studied. The experimental results depicted in Fig. 15 show that the interfacial potentials becomes more negative at lower $\mathrm{pH}$ and the potentials at higher $\mathrm{pH}$ are the same as in the absence of 2,4-D. The half-change occurs at $\mathrm{pH}$ comparable to the $\mathrm{pK}_{\mathrm{a}}$ of 2,4-D, which is about 2.6-2.8 $(99,100)$. This indicates that the observed changes of the interfacial potential difference are related to 2,4-D dissociation-association equilibrium, and that observation of more negative interfacial potential difference is related to the presence of neutral 2,4-D molecules.

In addition to 2,4-D concentration and $\mathrm{pH}$ dependence, the effect of salt concentration on the interfacial potential difference in the presence of 2,4-D was studied (Fig. 16). The effect of ionic strength was rather small, therefore two sets of measurements at $c_{2,4 D}=$ $1 \times 10^{-3} \mathrm{M}$ were compared: one at low ionic strength $(0.05-0.2 \mathrm{M})$ and one at high ionic strength $(1.0-2.5 \mathrm{M})$. For PC-chol monolayers, 


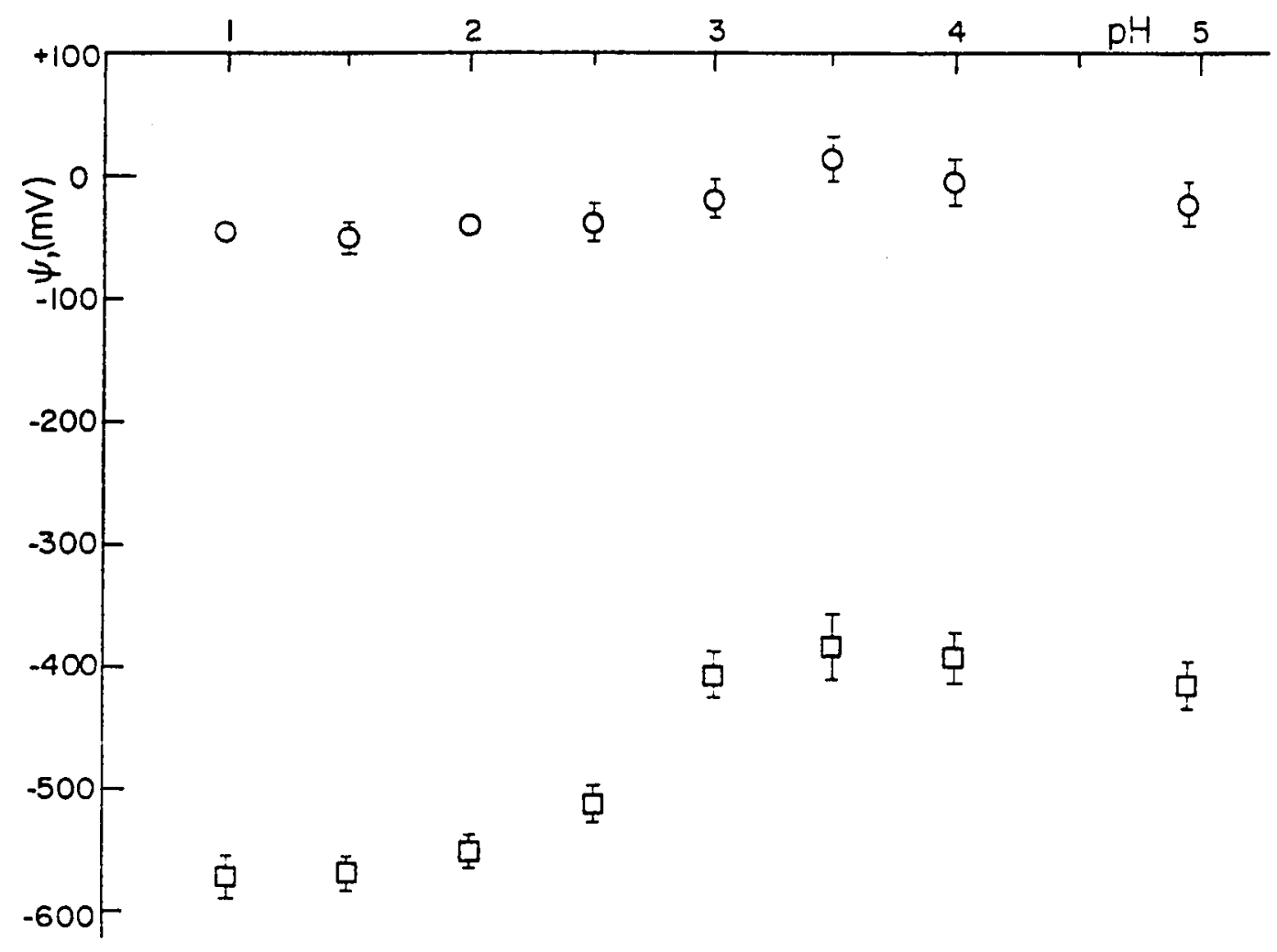

Figure 15. Changes of interfacial potential difference across the PC-chol monolayer (top) and the air/aqueous solution interface (bottom) as a function of $\mathrm{pH}$ (buffered). $\mathrm{c}_{\mathrm{KCl}}=0.5 \mathrm{M}, \mathrm{c}_{2,4-\mathrm{D}}=$ $1 \times 10^{-3} \mathrm{M}$. 


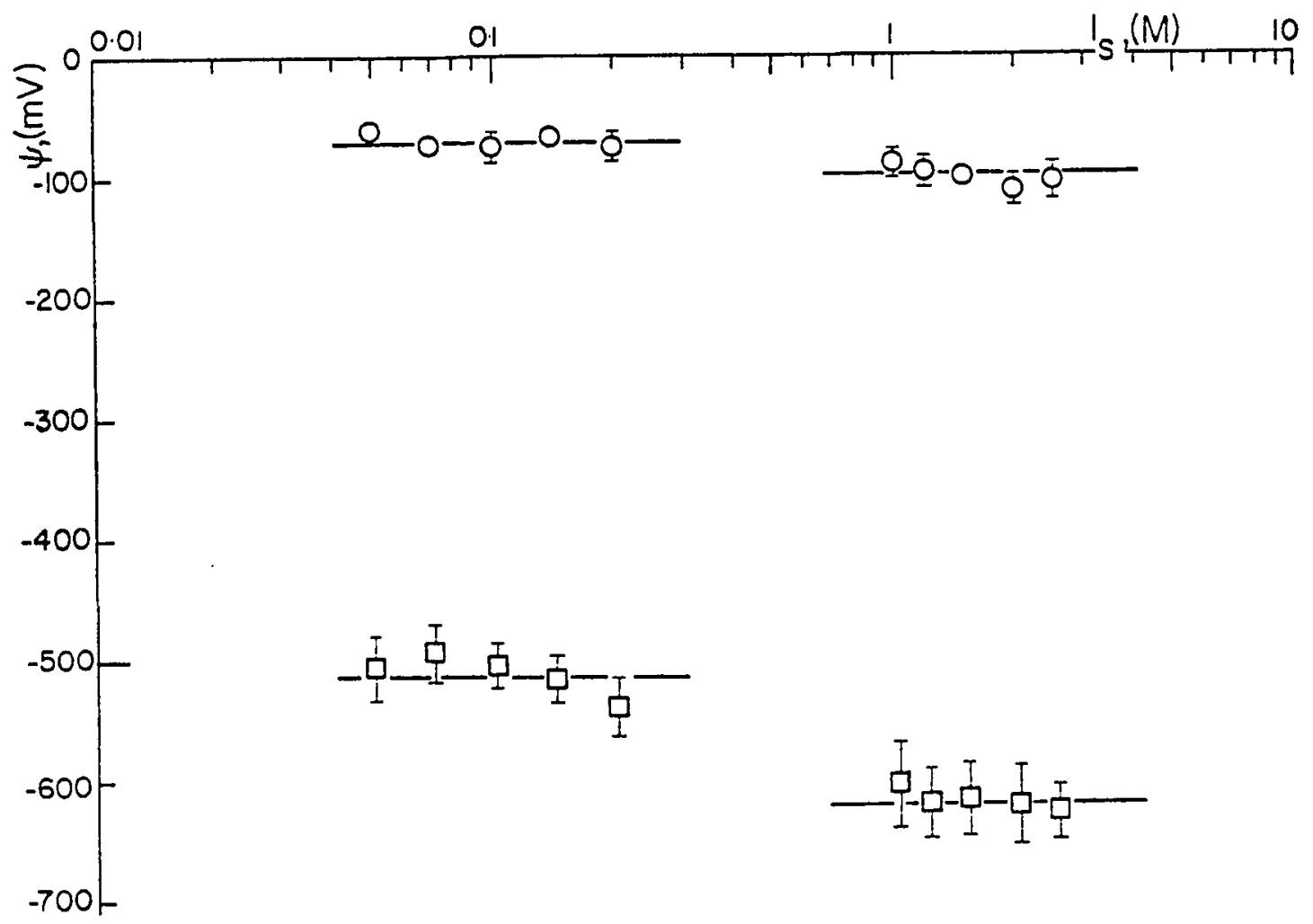

Figure 16. A comparison of interfacial potential difference across the PC-chol monolayer (top) and the air/aqueous solution interface (bottom) at high and low ionic strength. $\mathrm{pH}=2$ (buffered), $c_{2,4-D}=1 \times 10^{-3} \mathrm{M}$. Ionic strength was adjusted by $\mathrm{KCl}+\mathrm{LiCl}$, ratio $\mathrm{c}_{\mathrm{KCl}} / \mathrm{c}_{\mathrm{LiCl}}=1$. 
the interfacial potential differences are as follows: $-71 \pm 3 \mathrm{mV}$ at low and $-98 \pm 14 \mathrm{mV}$ at high ionic strength. For the air/water interface the effect is qualitatively similar: $-514 \pm 52 \mathrm{mV}$ at low ionic strength and $-622 \pm 13 \mathrm{mV}$ at high ionic strength. Thus, in either case the less polar side of the interface becomes more negative at high ionic strength. Similar kinds of measurements were done in GMO monolayers and the results are shown in Fig. 17. In the absence of 2,4-D the potential difference across GMO monolayer was found to be $318 \pm 9 \mathrm{mV}$ which agrees with 319 - $321 \mathrm{mV}$ reported by H1adky and Haydon (58). In contrast to PC-chol monolayers the electric potential of the hydrocarbon side of the GMO monolayer becomes significantly more regative in the presence of 2,4-D.

5g. EXPERIMENTS WITH AMINO ACID CONJUGATES OF 2,4-D

Amino acid conjugates of 2,4-D have been found to be physiologically active $(25,27)$. The purpose of this study is to establish their effect on electrical conductance of lipid bilayer membranes.

The following amino acid conjugates have been studied: Isoleucine 2,4-D, Leucine-2,4-D, Valine-2,4-D (Fig. 18). Dissociation characteristics have been determined by titrating with standard $\mathrm{NaOH}$ solution (99). The $\mathrm{pK}_{\mathrm{a}}$ value of these substances are all between 3.2 and 3.5 . For reasons which will be given later, the membrane conductivity measurements were done under two sets of conditions: (a) in the $\mathrm{pH}$ range 1-5 about 50 $\mu \mathrm{M}$ concentration of 2,4-D - amino acid conjugate in the aqueous medium was used, and (b) higher $(800 \mu \mathrm{M})$ concentration was used in the $\mathrm{pH}$ range 5-10. 


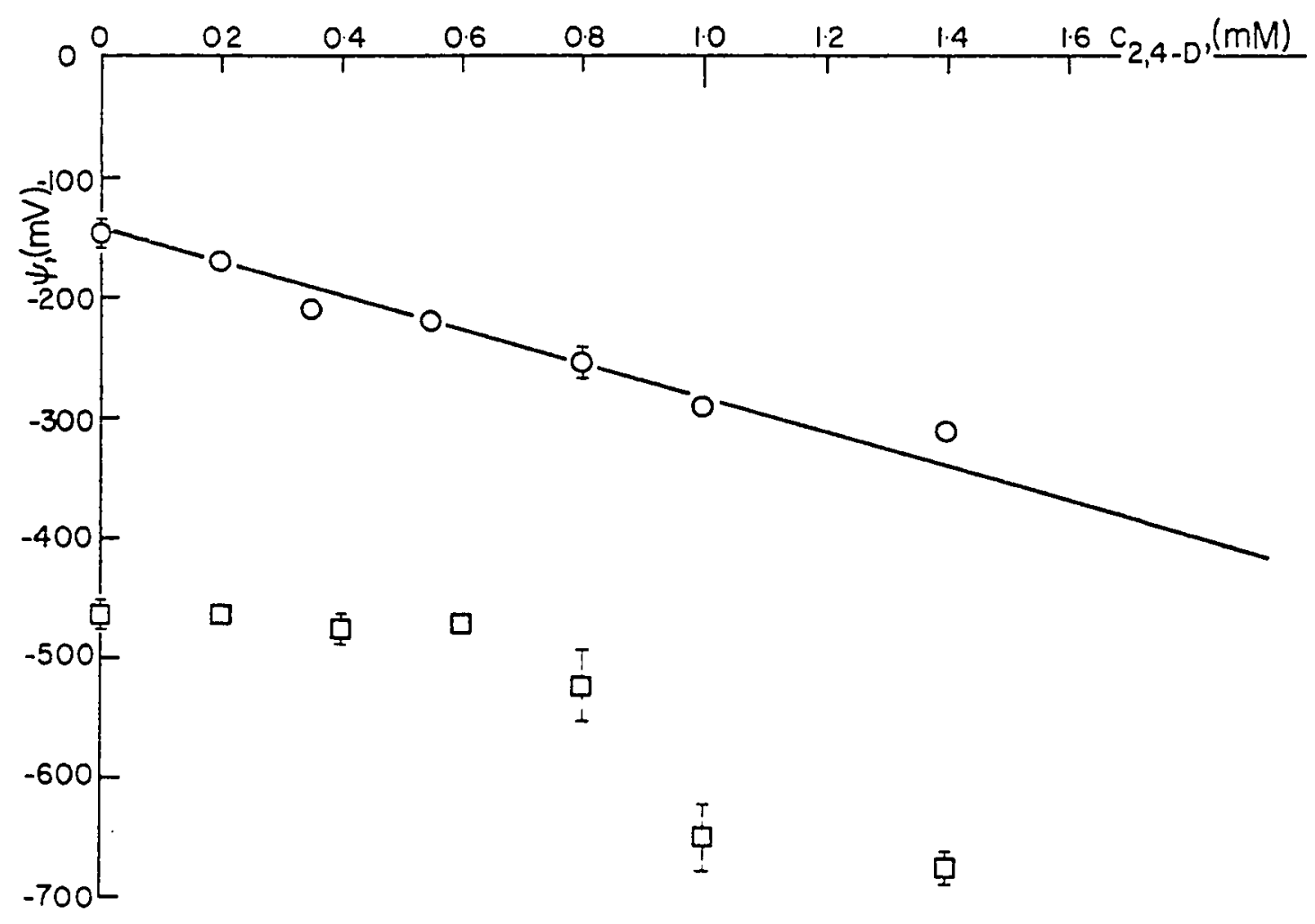

Figure 17. 2,4-D concentration dependence of interfacial potential difference across the cholesterol-free GMO monolayer (top) and the air/aqueous interface (bottom). $\mathrm{pH}=2$ (buffered), $c_{\mathrm{KCl}}=0.5 \mathrm{M}$. The solid line represent the least square fit of $\Delta \psi=b c_{2,4-D^{\circ}}$ 

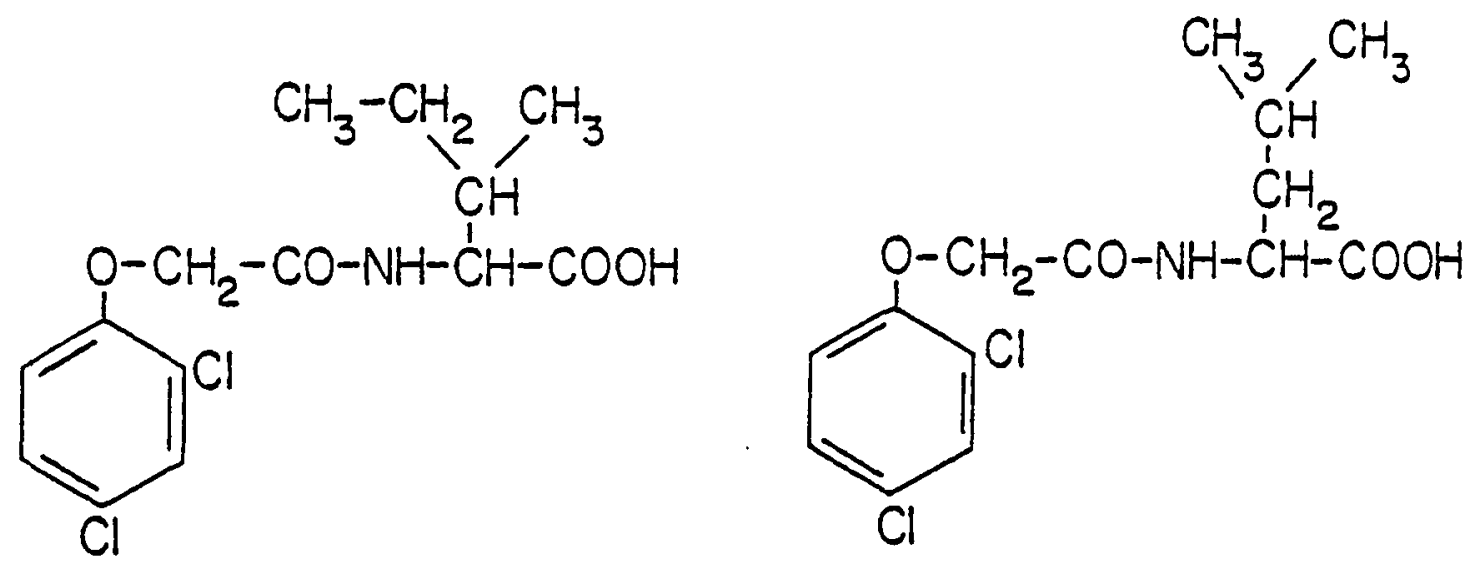

Isoleucine amino acid conjugate of $2,4-D$

Leucine amino acid conjugate of $2,4-D$

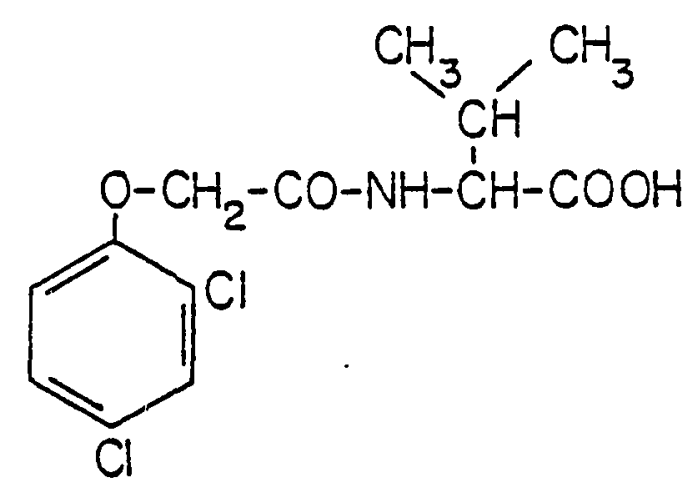

$$
\begin{aligned}
& \text { Valine amino acid } \\
& \text { conjugate of } 2,4-D
\end{aligned}
$$

Figure 18. The structures of Isolucine, Leucine, and Valine amino acid conjugates of $2,4-D$. 
We have found that these compounds do not induce membrane conductivity, which implies that their corresponding anions do not permeate through the membrane.

The ability of 2,4-D-amino acid conjugates to modify transport of membrane permeable ions was tested by means of nonactin-K ${ }^{+}$complex. The membranes were made electrically conductive in the presence of $K^{+}$ ion carrier nonactin, and the effect of 2,4-D-amino acid conjugates was determined by measuring the membrane conductance as a function of hydrogen ion concentration. The results shown in Figs. 19-21 indicate that there is a major enhancement of conductance at low $\mathrm{pH}$, i.e., when the amino acid conjugates are in the electrically neutral form. In addition to this major effect in the low $\mathrm{pH}$ range, a smaller but significant enhancement of membrane conductance was observed in the $\mathrm{pH}$ range 5-10 (Figs. 22-24). Because the latter effect is significantly smaller, it was necessary to use higher concentration $(800 \mu \mathrm{M})$ of $2,4-\mathrm{D}-$ amino acid conjugates. From their dissociation properties it follows that in the high $\mathrm{pH}$ range these compounds exist as anions. The enhancement of conductance, which takes place in the biologically interesting $\mathrm{pH}$ rarge, suggests that the anionic form of 2,4-D conjugates with amino acids also possess the ability to enhance transport of positive charged ions. An alternative explanation is that the enhancement effect at high $\mathrm{pH}$ is due to impurities in the samples of amino acid conjugates, which have not been eliminated and detected by conventional methods. One of the possible impurities is 2,4-dichlorophenol $(2,4-D C P)$ which has a $\mathrm{pK}_{\mathrm{a}}$ value of 7.8 (101). Its presence could explain the existence of high $\mathrm{pH}$ enhancement effect, especially since the effect disappears at $\mathrm{pH}$ exceeding 8 , that is when $2,4-\mathrm{DCP}$ becomes ionized. The 


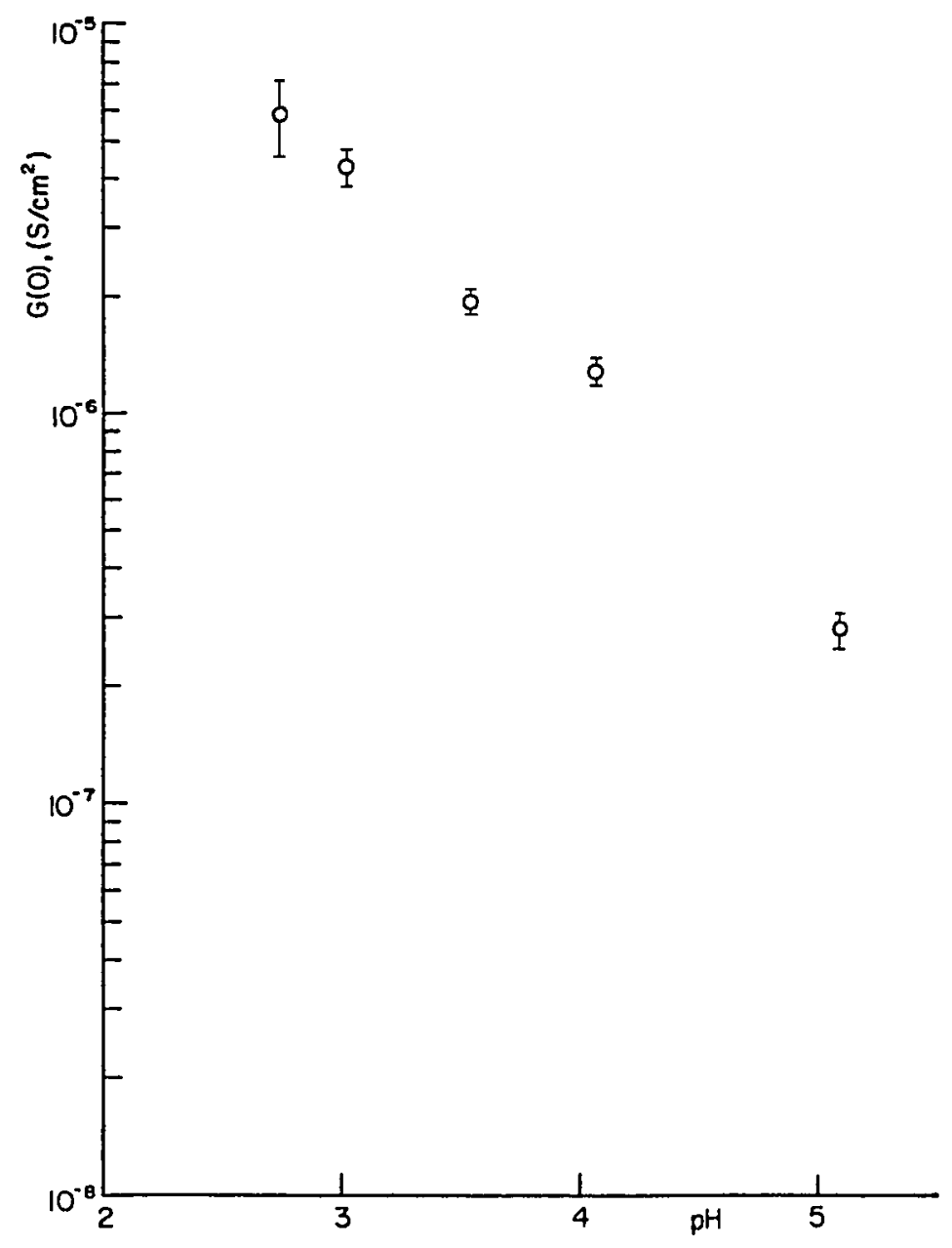

Figure 19. $\mathrm{pH}$ dependence of 2,4-D-Leu amino acid conjugate enhanced nonactin- $\mathrm{K}^{+}$conductance of $\mathrm{PC}-\mathrm{chol}$ membranes in the low $\mathrm{pH}$ range. $c_{2,4-D-\text { Leu }}=5 \times 10^{-5} \mathrm{M}, c_{\text {nonactin }}$ (aqueous sol.) $=1 \times 10^{-7} \mathrm{M}$, $c_{\text {nonactin }}$ (membrane sol.) $=3 \times 10^{-5} \mathrm{M}, c_{\mathrm{K}^{+}}=0.1 \mathrm{M}$, ionic strength $(\mathrm{LiCl}+\mathrm{KCl})=0.5 \mathrm{M}$. 


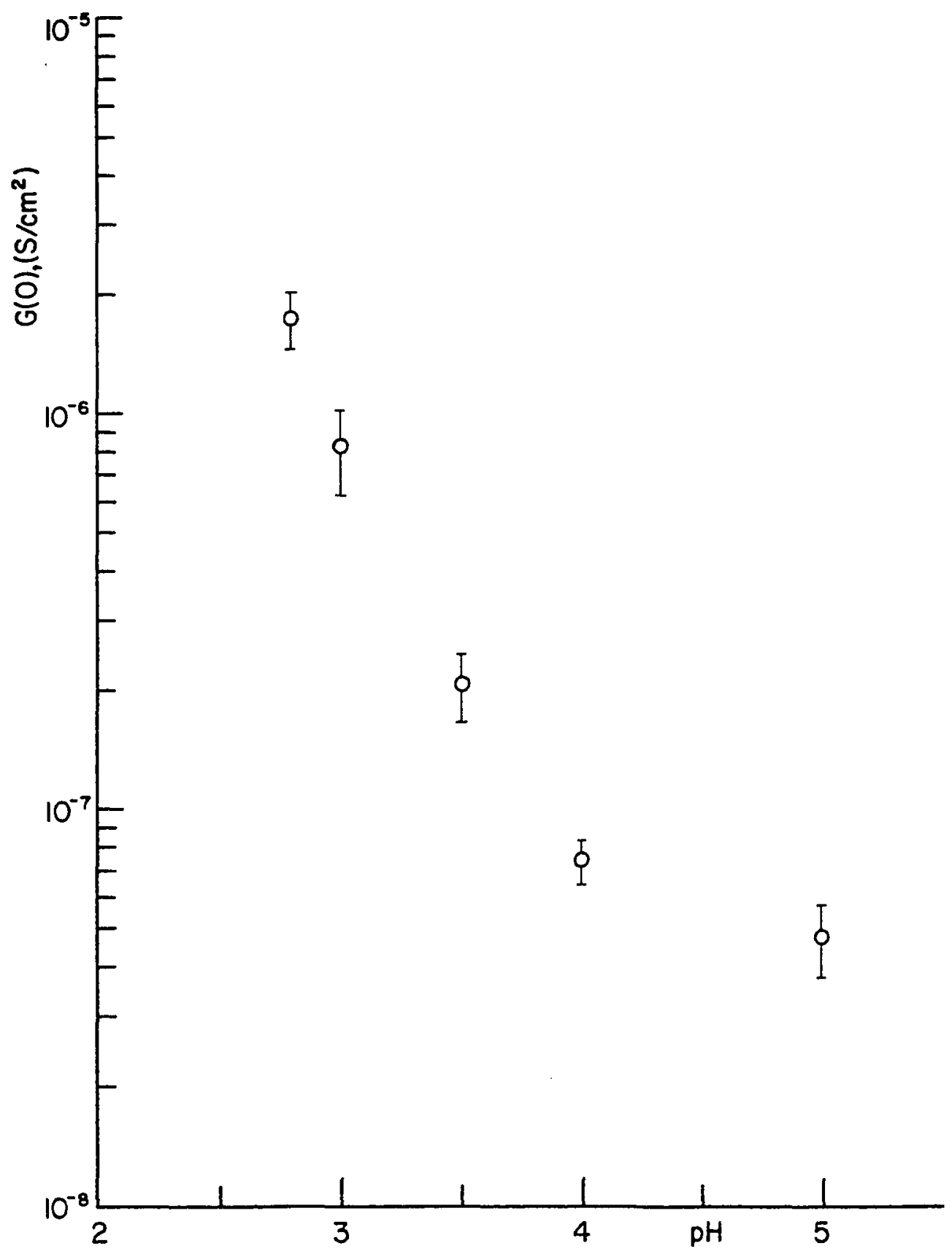

Figure 20. $\mathrm{pH}$ dependence of the 2,4-D-Ile amino acid conjugate enhanced nonactin- $\mathrm{K}^{+}$conductance of $\mathrm{PC}-\mathrm{chol}$ membranes in the low $\mathrm{pH}$ range. $c_{2,4-D-I l e}=4 \times 10^{-5} \mathrm{M}, c_{\text {nonactin }}$ (aqueous sol.) $=$ $1 \times 10^{-7} \mathrm{M}, c_{\text {nonactin }}$ (membrane sol.) $=3 \times 10^{-5} \mathrm{M}, c_{\mathrm{K}^{+}}=0.1 \mathrm{M}$, ionic strength $(\mathrm{LiC} 1+\mathrm{KC} 1)=0.5 \mathrm{M}$. 


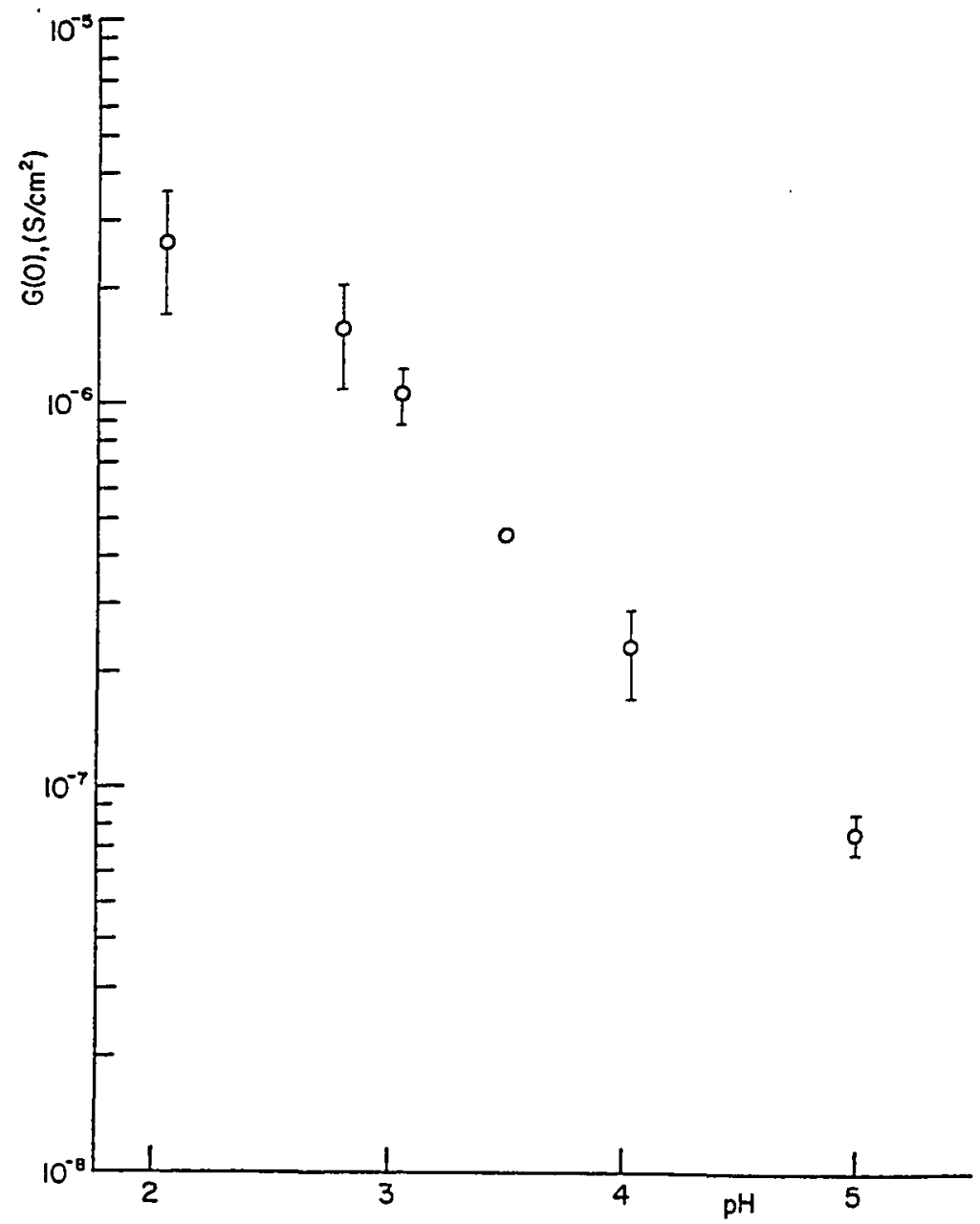

Figure 21. $\mathrm{pH}$ dependence of the 2,4-D-Val amino acid conjugate enhanced nonactin- $\mathrm{K}^{+}$conductance of $\mathrm{PC}-\mathrm{chol}$ membranes in the low $\mathrm{pH}$ range. $c_{2,4-\mathrm{D}-\mathrm{Val}}=7 \times 10^{-5} \mathrm{M}, \mathrm{c}_{\text {nonactin }}$ (aqueous sol.) $=1 \times 10^{-7} \mathrm{M}, c_{\text {nonactin }}$ (membrane sol.) $=3 \times 10^{-5} \mathrm{M}, c_{\mathrm{K}^{+}}=0.1 \mathrm{M}$, ionic strength $(\mathrm{LiCl}+\mathrm{KCl})=0.5 \mathrm{M}$. 


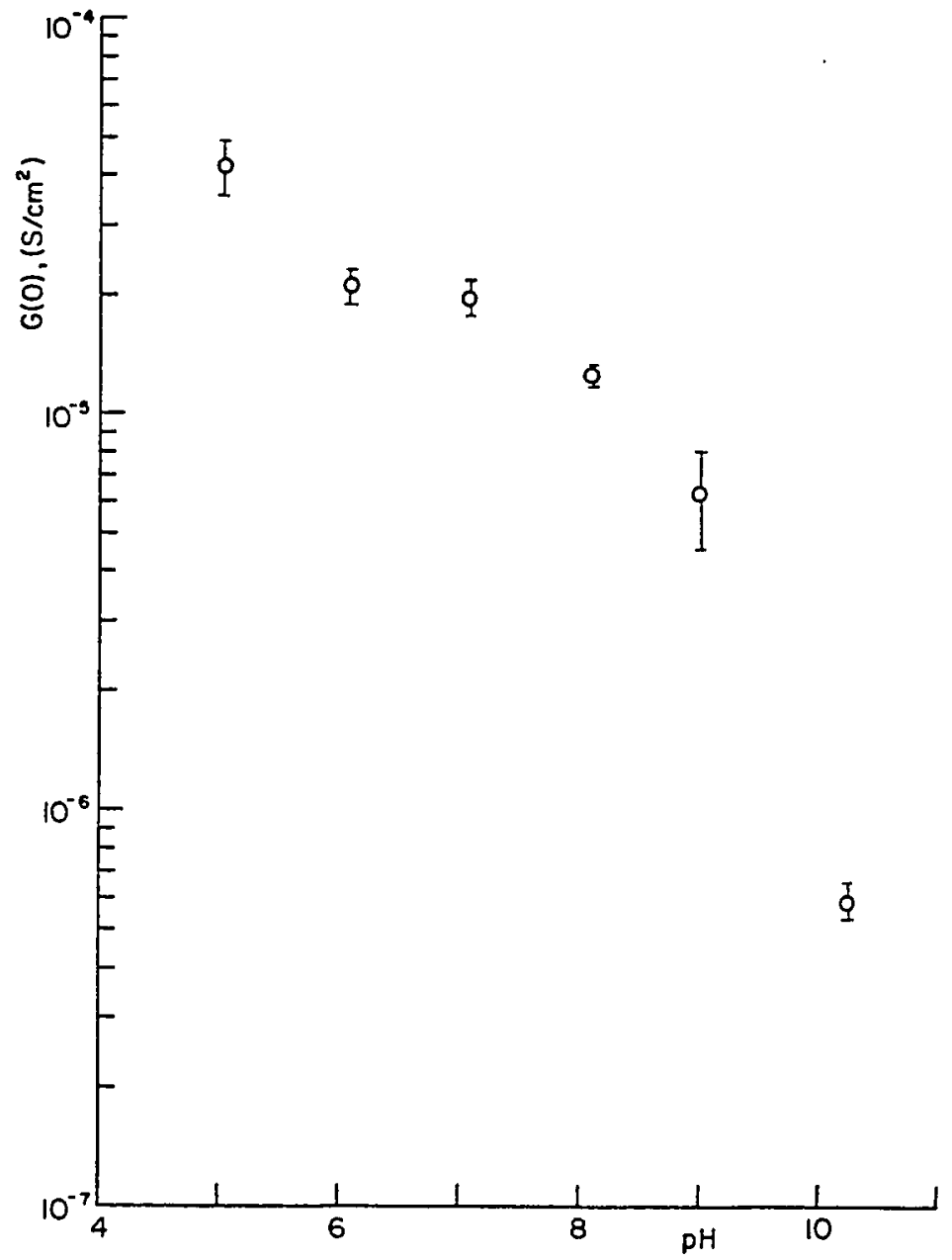

Figure 22. $\mathrm{pH}$ dependence of 2,4-D-Leu amino amino acid conjugate enhanced nonactin- $\mathrm{K}^{+}$conductance of $\mathrm{PC}-\mathrm{chol}$ membranes in the high $\mathrm{pH}$ range. $c_{2,4-D-L e u}=8 \times 10^{-4} \mathrm{M}, c_{\text {nonactin }}$ (aqueous sol.) $=$ $1 \times 10^{-7} \mathrm{M}, c_{\text {nonactin }}$ (membrane sol.) $=3 \times 10^{-5} \mathrm{M}, c_{\mathrm{K}^{+}}=0.1 \mathrm{M}$, ionic strength $(\mathrm{LiCl}+\mathrm{KCl})=0.5 \mathrm{M}$. 


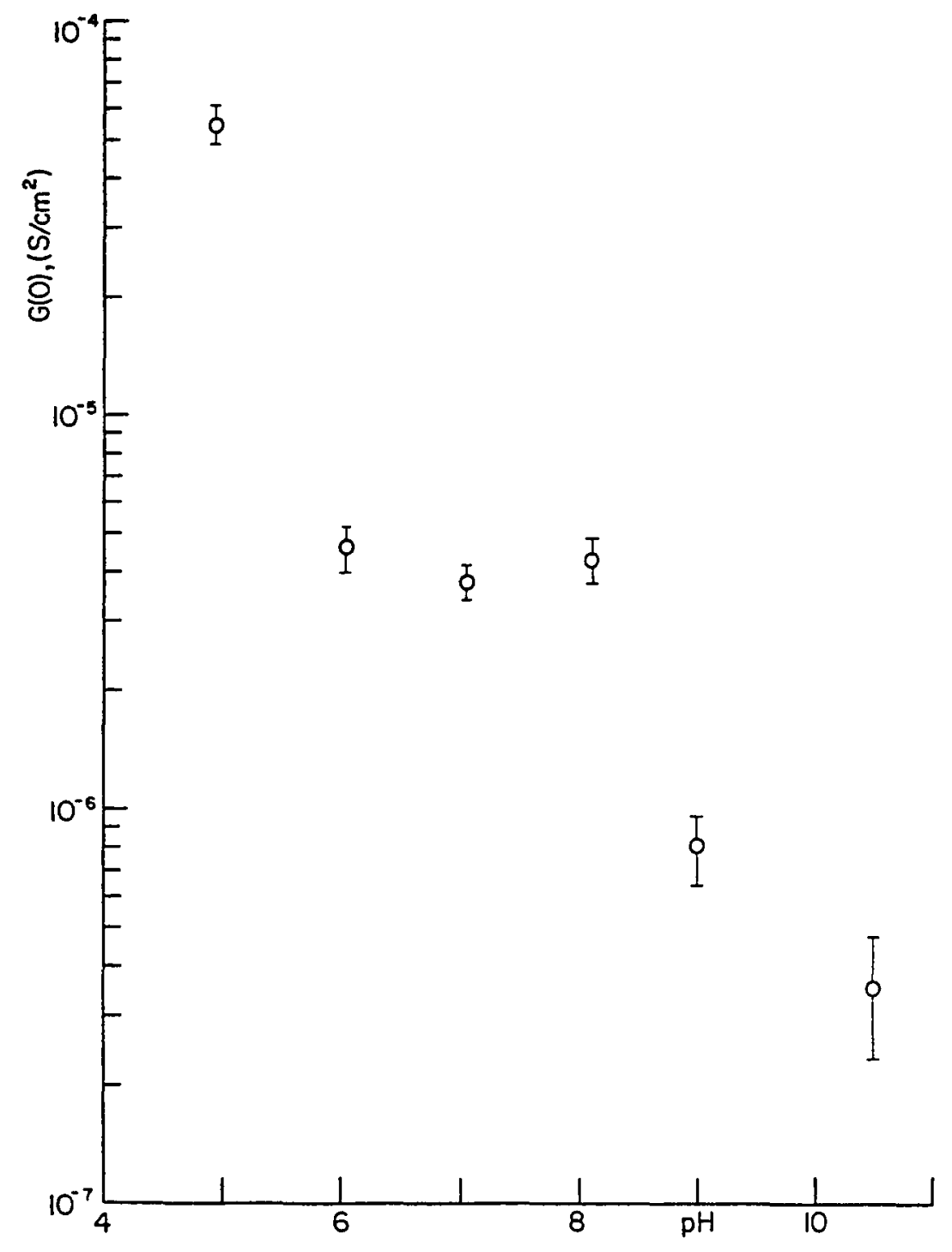

Figure 23. $\mathrm{pH}$ dependence of 2,4-D-Ile amino acid conjugate enhanced nonactin- $\mathrm{K}^{+}$conductance of $\mathrm{PC}-\mathrm{chol}$ membranes in the high $\mathrm{pH}$ range. $\mathrm{c}_{2,4-\mathrm{D}-\mathrm{Ile}}=8 \times 10^{-4} \mathrm{M}, \mathrm{c}_{\text {nonactin }}$ (aqueous sol.) $=$ $1 \times 10^{-7} \mathrm{M}, c_{\text {nonactin }}$ (membrane sol.) $=3 \times 10^{-5} \mathrm{M}, c_{\mathrm{K}^{+}}=0.1 \mathrm{M}$, ionic strength $(\mathrm{LiCl}+\mathrm{KCl})=0.5 \mathrm{M}$. 


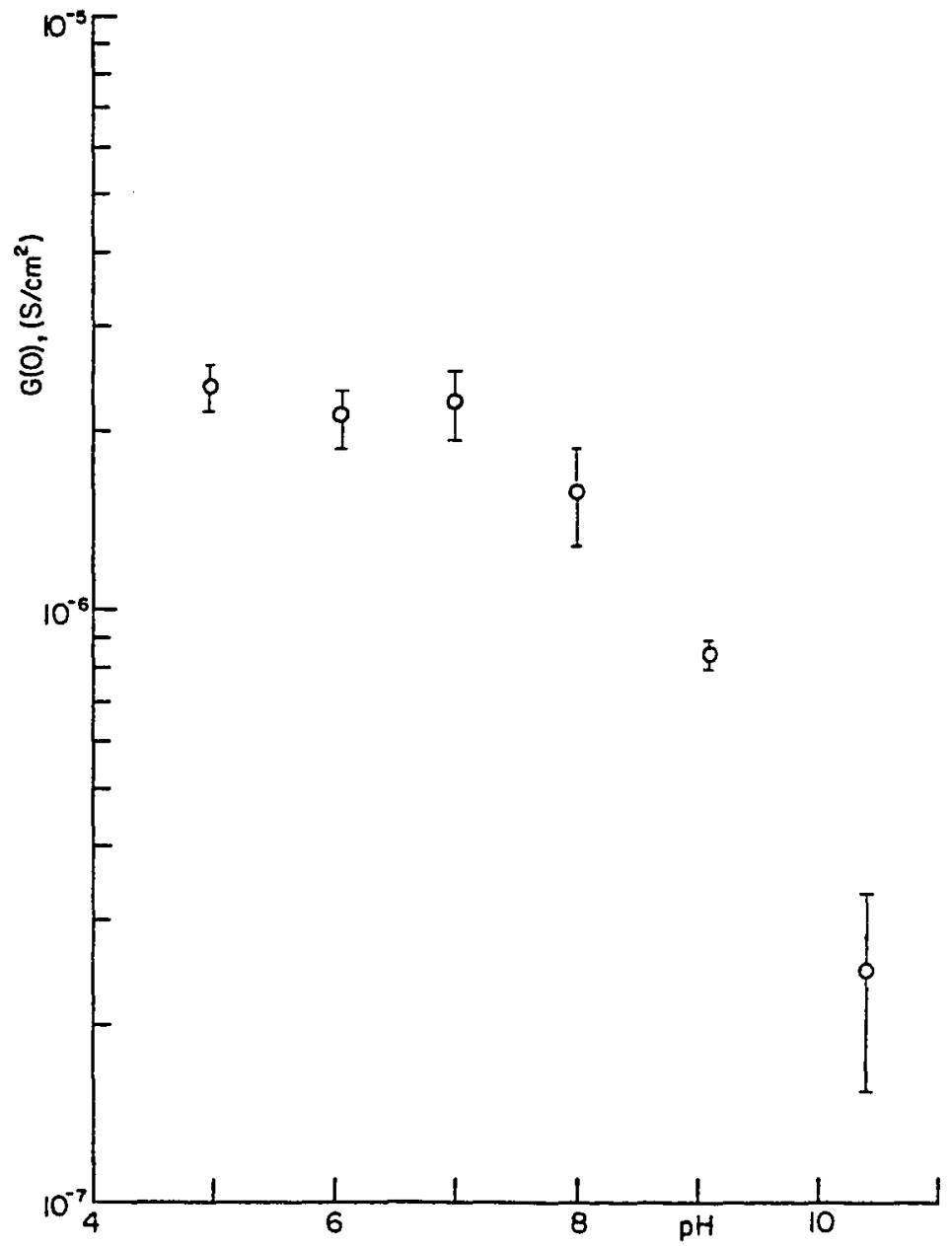

Figure 24. $\mathrm{pH}$ dependence of 2,4-D-Val amino acid conjugate enhanced nonactin- $\mathrm{K}^{+}$conductance of $\mathrm{PC}-\mathrm{chol}$ membranes in the high $\mathrm{pH}$ range. $\mathrm{c}_{2,4-\mathrm{D}-\mathrm{Val}}=8 \times 10^{-4} \mathrm{M}, \mathrm{c}_{\text {nonactin }}$ (aqeous sol.) $=$ $1 \times 10^{-7} \mathrm{M}, c_{\text {nonactin }}$ (membrane sol.) $=3 \times 10^{-5} \mathrm{M}, c_{\mathrm{K}^{+}}=0.1 \mathrm{M}$, ionic strength $(\mathrm{LiCl}+\mathrm{KCl})=0.5 \mathrm{M}$. 


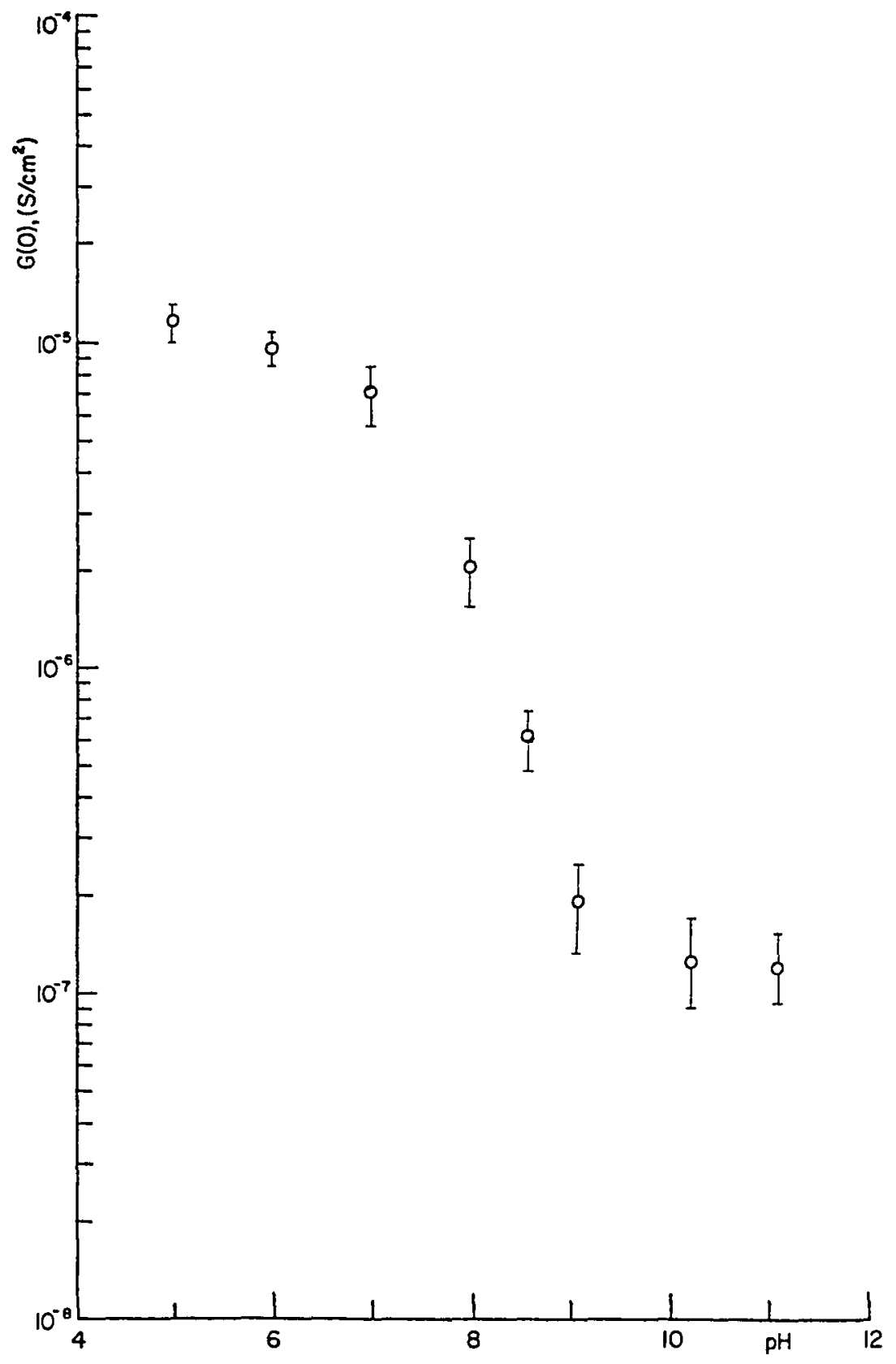

Figure 25. Effect of $\mathrm{pH}$ on the 2,4-DCP enhanced nonactin- $\mathrm{K}^{+}$ conductance. The data points represent the conductance after subtraction of the conductance of 2,4-DCP itself. $c_{2,4-D C P}=$ $1 \times 10^{-4} \mathrm{M}, c_{\text {nonactin }}$ (aqueous sol.) $=1 \times 10^{-7} \mathrm{M}, \mathrm{c}_{\text {nonactin }}$ (membrane sol.) $=3 \times 10^{-5} \mathrm{M}, \mathrm{c}_{\mathrm{K}^{+}}=0.1 \mathrm{M}$, ionic strength ( $\mathrm{LiCl}+$ $\mathrm{KCl})=0.5 \mathrm{M}$. 
results of studies with 2,4-DCP are shown in Fig. 25. Since 2,4-DCP induces membrane conductivity due to its own presence, in addition to enhancement of nonactin- $\mathrm{K}^{+}$conductivity, the data in Fig. 25 have been corrected for the 2,4-DCP induced conductance. The results will be discussed in the following chapter.

5h. EXPERIMENTS WITH NATURAL GROWTH HORMONE INDOLE ACETIC ACID

Since 2,4-D and other phenoxy pesticides act as auxins at low concentrations (14) it is of interest to investigate the effect of the natural auxin indole acetic acid (IAA, see Fig. 26) on ion transport in lipid membranes.

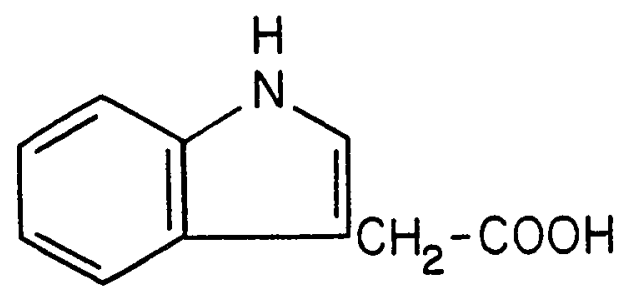

Figure 26. The structure of indole acetic acid.

The current voltage characteristics of bilayer lipid membranes were measured in the presence of IAA in the aqueous solution. IAA concentration was varied from zero to $1 \mathrm{mM}$ and $\mathrm{pH}$ from 2 to 7 . The $\mathrm{pK}_{\mathrm{a}}$ of IAA is 4.75 (102). Under these conditions IAA did not change the conductance of unmodified lipid membranes in the experimental error. To investigate the possibility of changing the membrane permeability of other ions by IAA, nonactin $-\mathrm{K}^{+}$complex was used as a membrane probe as before. The IAA enhanced conductance of nonactin- $\mathrm{K}^{+}$complex as a function of $\mathrm{pH}$ was measured and the results shown in Fig. 27. 


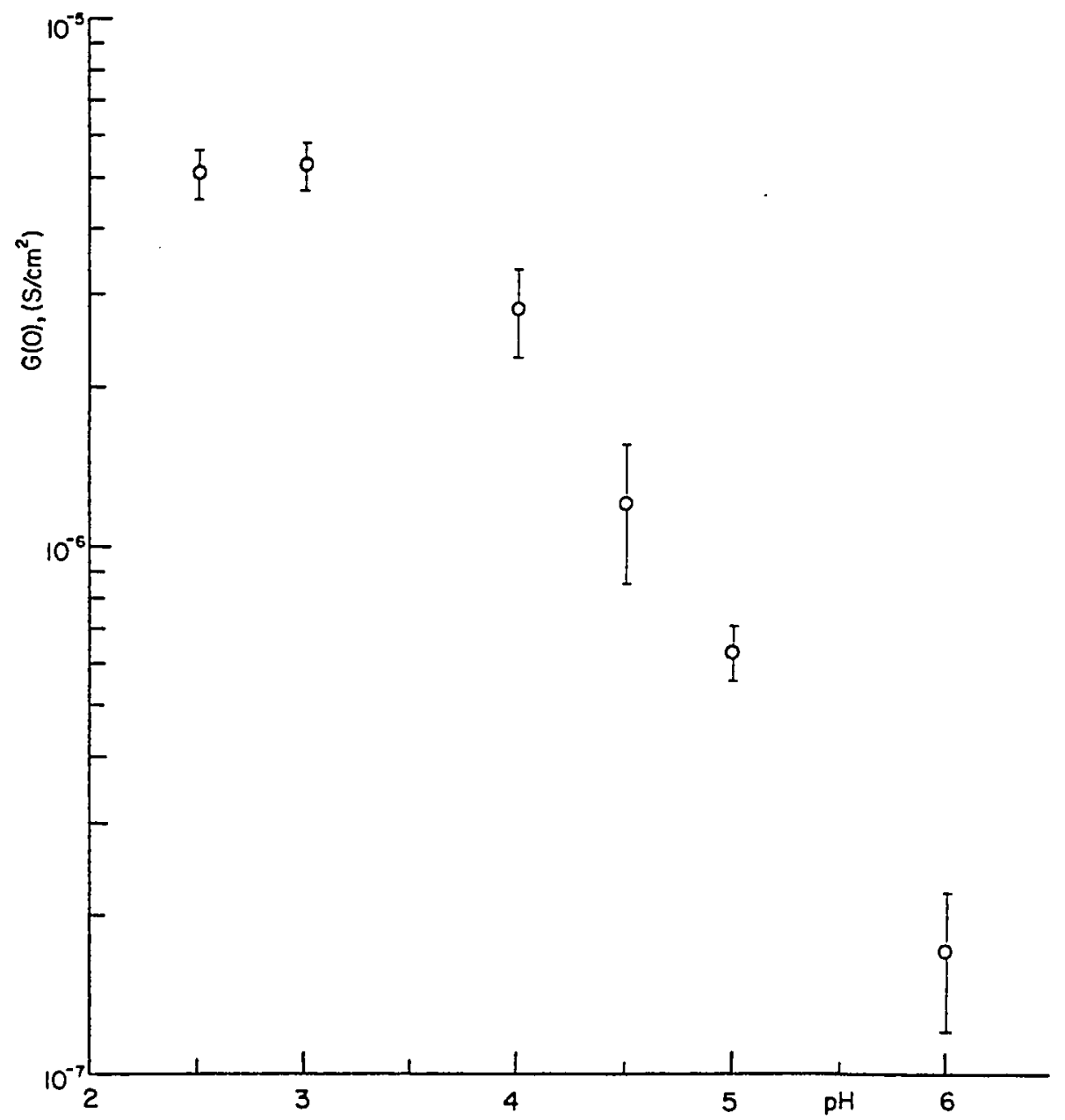

Figure 27. $\mathrm{pH}$ dependence of IAA enhanced nonactin- $\mathrm{K}^{+}$conductance of PC-chol membranes. $c_{I A A}=1 \times 10^{-3} \mathrm{M}, c_{\text {nonactin }}$ (aqueous sol.) $=3 \times 10^{-7} \mathrm{M}, c_{\text {nonactin }}$ (membrane sol.) $=1 \times 10^{-4} \mathrm{M}, \mathrm{c}_{\mathrm{K}^{+}}=0.1 \mathrm{M}$, ionic strength $(\mathrm{LiCl}+\mathrm{KCl})=0.5 \mathrm{M}$. 
The results indicate that the enhancement is small at $\mathrm{pH}>\mathrm{pK}_{\mathrm{a}}\left(\mathrm{pK}_{\mathrm{a}}=\right.$ 4.75), and the enhancement is more pronounced at $\mathrm{pH}^{2} \mathrm{pK}_{a}$, i.e., when IAA is mainly in the neutral form. Thus the effect of natural auxin on transport of cations is similar to that of 2,4-D and 2,4-D-amino acid conjugates.

5i. EXPERIMENTS WITH 2,4-DICHLOROPHENOXYBUTYRIC ACID $(2,4$-DB $), 2,4,5-$ TRICHLOROPHENOXYACETIC ACID $(2,4,5-\mathrm{T}), 2,4,6$-TRICHLOROPHENOXYACETIC ACID $(2,4,6-T)$ AND 2,4-D - ISOBUTYL ESTER $(2,4-D-I B E)$

The purpose of this series of experiments was to investigate the effect of different, but structurally related, phenoxy compounds on the ionic permeability of bilayer lipid membranes, 2,4-DB and 2,4,5-T are also very active herbicides, as is $2,4-D$, whereas $2,4,6-T$ has very little or no herbicidal activity $(103,104) \cdot 2,4-D$ - isobutyl ester is also one of the esters of 2,4-D which is used in agriculture as a herbicide. Molecular structures of these compounds are shown in Fig. 28.

The conductivity of PC-chol membranes were measured in a wide $\mathrm{pH}$ range with the above compounds present in the aqueous solution. The measurements revealed that none of these compounds induce the conductance of unmodified PC-chol membranes significantly. This observation confirmed our expectation, since 2,4-D by itself also did not change the conductance of lipid membranes.

To investigate the changes of the conductance due to positively charged ions, nonactin- $\mathrm{K}^{+}$complex was used as a probe, as before. The nonactin- $\mathrm{K}^{+}$conductance in the presence of $2,4-\mathrm{DB}$ and $2,4,5-\mathrm{T}$ as 


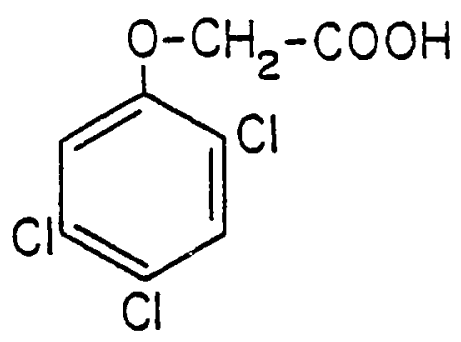

$2,4,5-T$

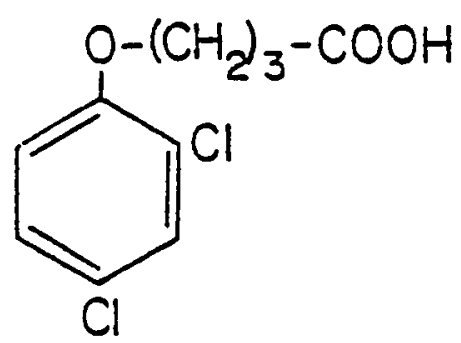

$2,4-\mathrm{DB}$

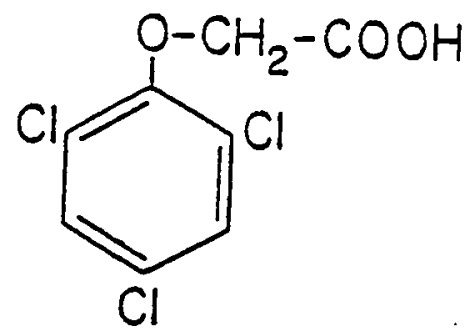

$2,4,6-T$

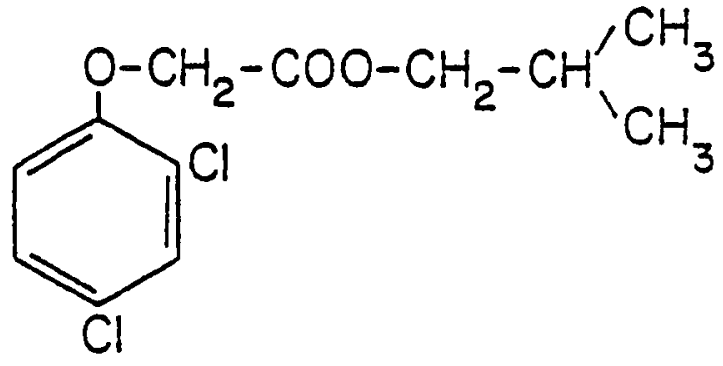

2,4-D-isobutyl ester

Figure 28. The structures of $2,4,5-T, 2,4,6-T, 2,4-D B$ and 2,4-D-isobutyl ester $(2,4-D-I B E)$. 
a function of $\mathrm{pH}$ is shown in Figs. 29 and 30 . The results indicate that (a) the nonactin- $\mathrm{K}^{+}$conductance is enhanced in the presence of 2,4-DB and 2,4,5-T and, (b) the enhancement is more prominent at $10 \mathrm{w} \mathrm{pH}$, at $\mathrm{pH}<\mathrm{pK}_{\mathrm{a}}$, that is again, when these compunds are predominantly in the neutral form. As the $\mathrm{pH}$ increases, the enhancement becomes small. The $\mathrm{pK}_{\mathrm{a}}{ }^{\prime} \mathrm{s}$ of $2,4-\mathrm{DB}$ and $2,4,5-\mathrm{T}$ are 4.58 and $2.6-2.8(100,105,106)$, respectively. The effect is actually similar to that observed for $2,4-D$.

Interesting results have been obtained with $2,4,5-\mathrm{T}$ and $2,4,6-\mathrm{T}$. These results are presented in Fig. 31. From their comparison it follows that $2,4,5-T$ enhanced membrane conductance is significantly greater than that due to $2,4,6-\mathrm{T}$. As it has been mentioned above, 2,4,5-T is also biologically more active than 2,4,6-T. The enhancement of membrane conductance by $2,4,5-T$ is accompanied by changes of voltage dependence of membrane conductance in a manner similar to that described for 2,4-D (Sec. 5b.1, and Fig. 8b).

The membrane conductivity studies with 2,4-D-IBE were done differently. This compound is not soluble in water, and was therefore incorporated into the membrane-forming solution. The membrane conductivity effect of this compound is absent. For $10 \mathrm{mM}$ concentration of 2,4-D-IBE in the membrane-forming solution, neither the conductivity of unmodified membrane, nor the conductivity due to the membrane probe, nonactin- $\mathrm{K}^{+}$complex, changed significantly. 


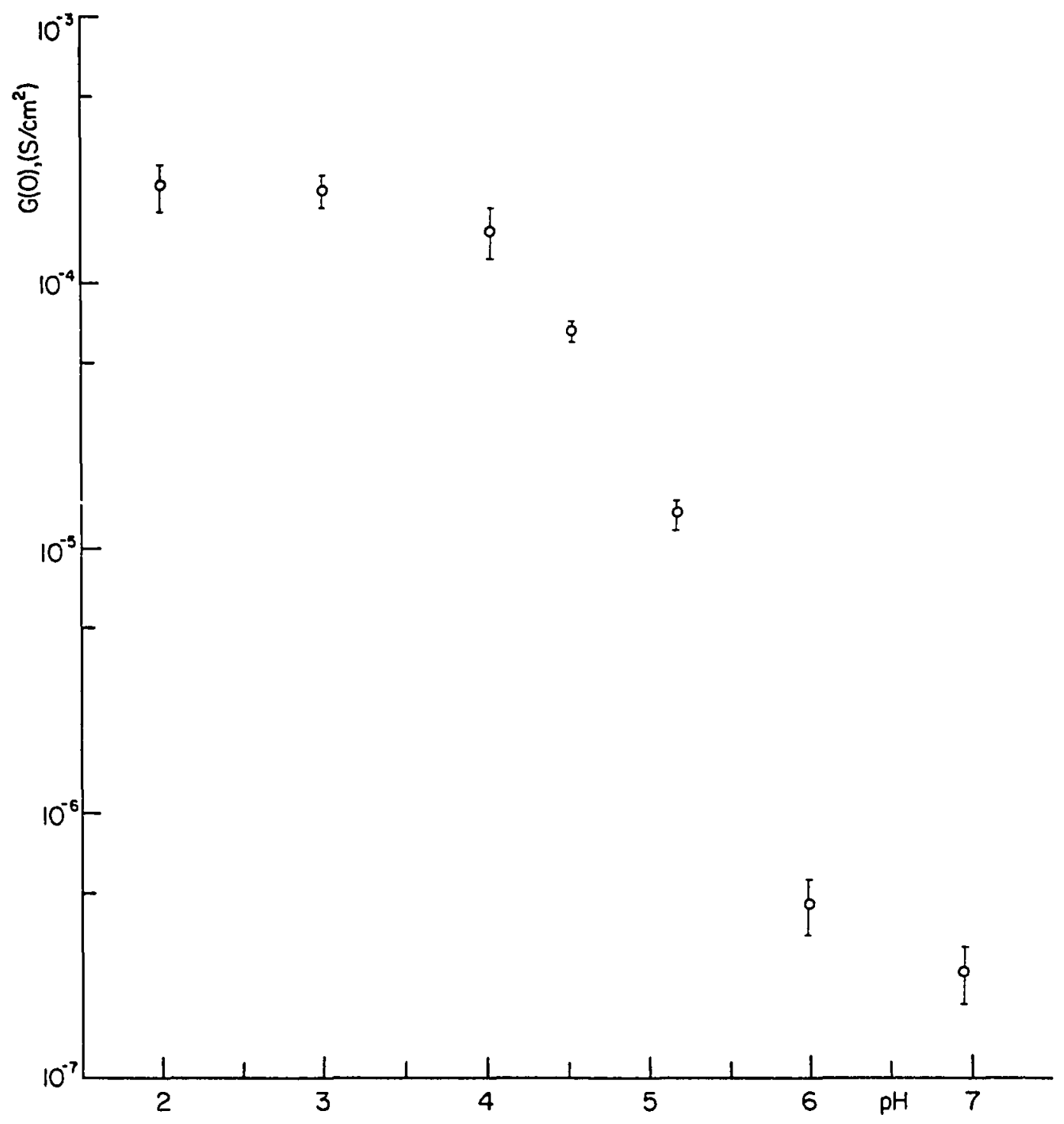

Figure 29. $\mathrm{pH}$ dependence of 2,4-DB enhanced nonactin- $\mathrm{K}^{+}$conductance. $c_{2,4-D B}=8 \times 10^{-5} \mathrm{M}, c_{\text {nonactin }}$ (aqueous solution) $=$ $3 \times 10^{-7} \mathrm{M}, \mathrm{c}_{\text {nonactin }}$ (membrane solution) $=1 \times 10^{-4} \mathrm{M}, \mathrm{c}_{\mathrm{K}^{+}}=$ $0.06 \mathrm{M}$, ionic strength $(\mathrm{LiCl}+\mathrm{KCl})=1 \mathrm{M}$. 


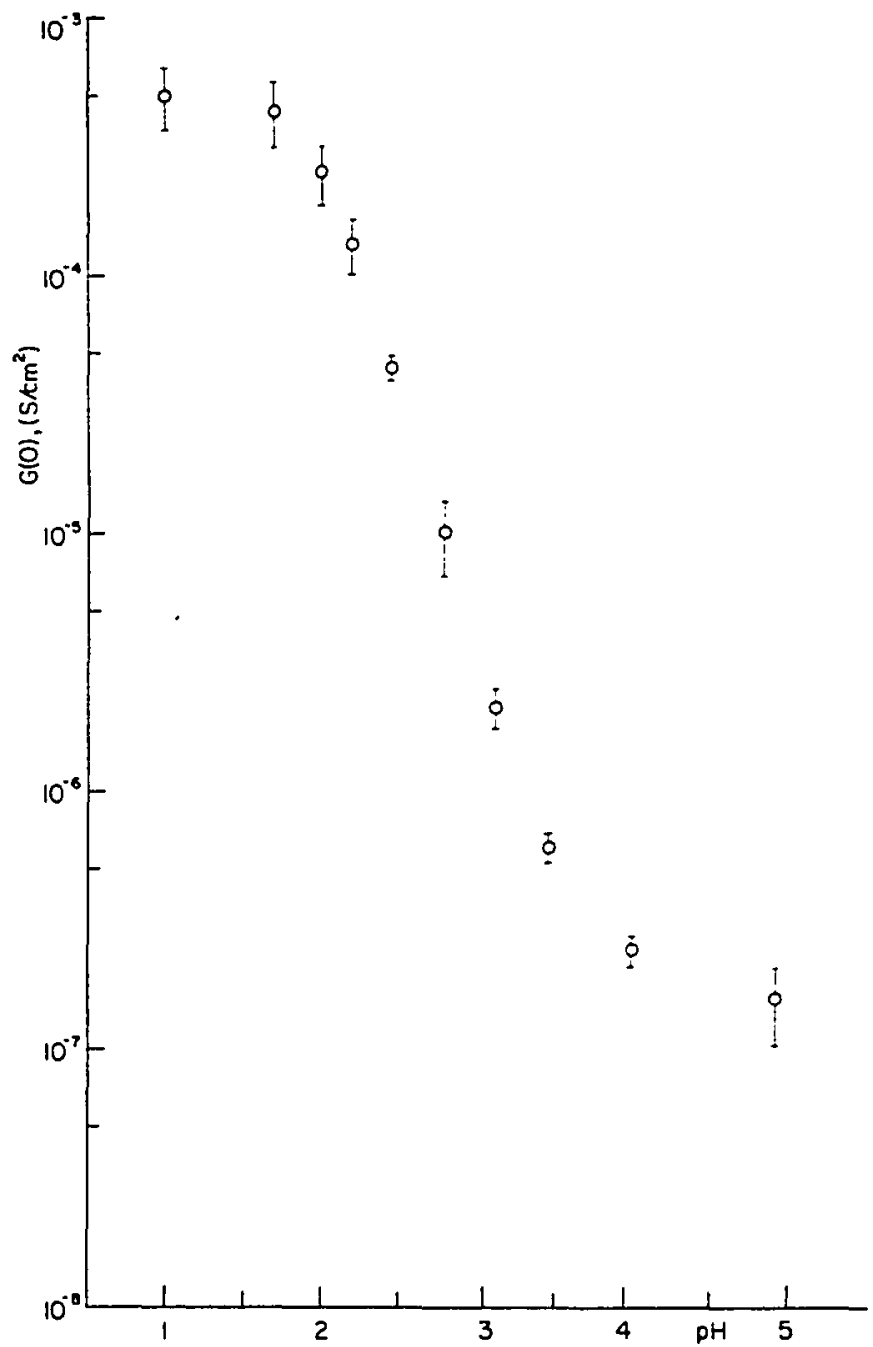

Figure 30. $\mathrm{pH}$ dependence of $2,4,5-\mathrm{T}$ enhanced nonactin- $\mathrm{K}^{+}$ conductance. $c_{2,4,5-T}=1.2 \times 10^{-4} \mathrm{M}, c_{\text {nonactin }}$ (aqueous solution) $=3 \times 10^{-7} \mathrm{M}, c_{\text {nonactin }}$ (membrane solution) $=1 \times 10^{-4} \mathrm{M}$, $\mathrm{c}_{\mathrm{K}^{+}}=0.06 \mathrm{M}$, ionic strength $(\mathrm{LiCl}+\mathrm{KCl})=1 \mathrm{M}$. 


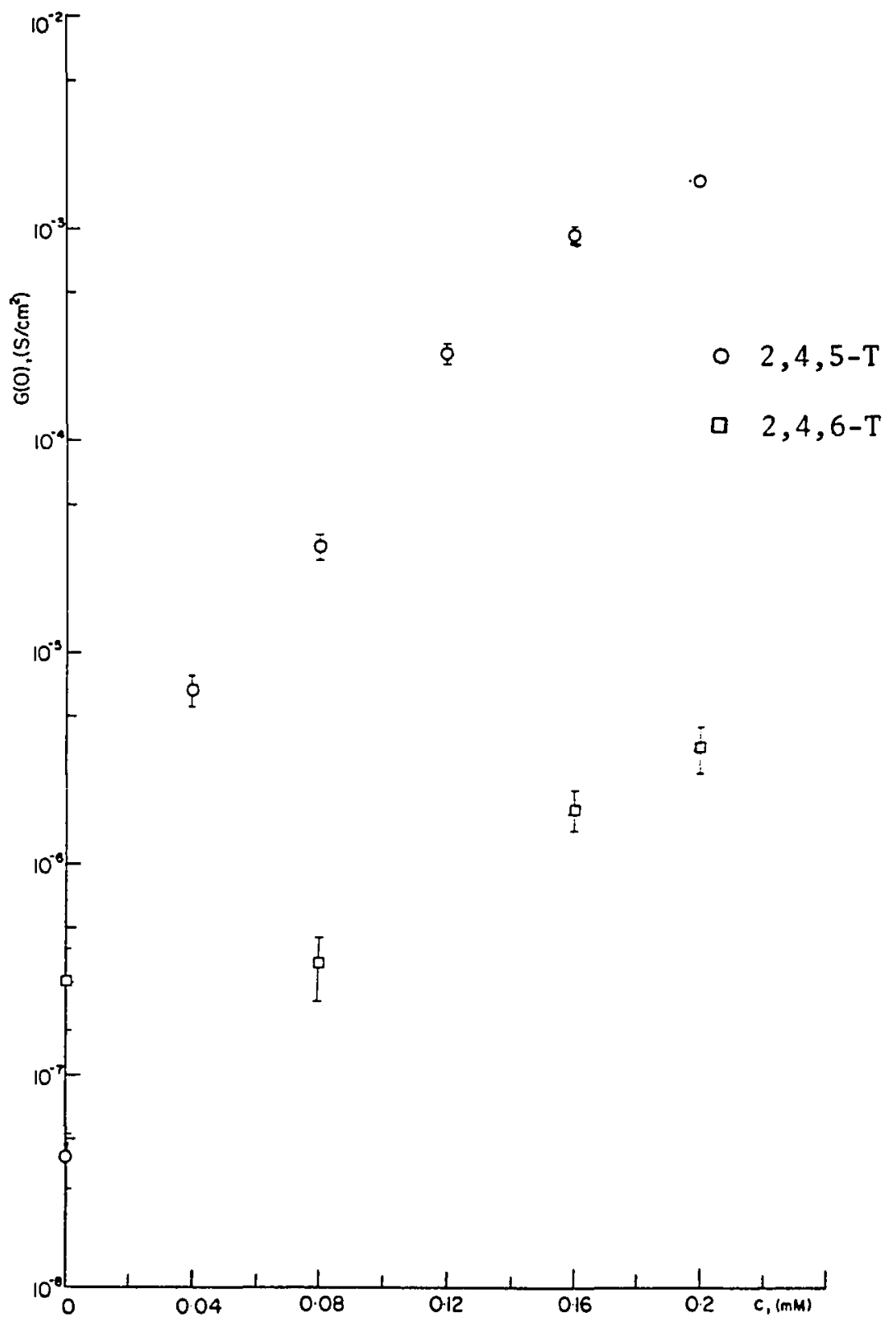

Figure 31. Effect of 2,4,5-T and 2,4,6-T on the nonactin- $\mathrm{K}^{+}$ conductance. $c_{\text {nonactin }}$ (aqueous solution) $=1 \times 10^{-7} \mathrm{M}$, $c_{\text {nonactin }}$ (membrane solution) $=3 \times 10^{-5} \mathrm{M}, \mathrm{c}_{\mathrm{K}+}=0.06 \mathrm{M}$, ionic strength $(\mathrm{LiCl}+\mathrm{KC} 1)=1 \mathrm{M}, \mathrm{pH}=2$ (buffered). 
CHAPTER VI

ANALYSIS AND DISCUSSION OF EXPERIMENTAL RESULTS

6a. MEMBRANE CONDUCTANCE INDUCED BY PHENOXY ACIDS, AMINO ACID CONJUGATES OF 2,4-D AND INDOLE ACETIC ACID

Membrane conductivity measurements have indicated that the ionized form of these compounds are membrane impermeable and that their action is in principle different from that of uncouplers of oxidative phosphorylation. Membrane impermeability of ionized form of phenoxy pesticides is very likely related to localization of charge on the carboxyl group $-\mathrm{COO}^{-}$. This follows from the concept of membrane as a layer of dielectric (Eq. 1). The membrane permeable ions, $\mathrm{TPhB}^{-}$and $\mathrm{TPhAs}^{+}$, have in contrast, the excess charge delocalized within the molecule consisting of four benzene rings. Thus the effective ionic radius is much greater than that of phenoxy-type anions.

6b. EFFECT OF 2,4-D ON KINETICS OF CARRIER-MEDIATED POTASSIUM ION TRANSPORT ACROSS MEMBRANE

The primary effect of 2,4-D was found to be the changes in the transmembrane ion transport of other ions. As shown in Fig. 5, in the presence of $2,4-D$, the conductance due to cations nonactin- $\mathrm{K}^{+}$complex and $\mathrm{TPhAs}^{+}$was enhanced while the conductance of negatively charged $\mathrm{TPhB}^{-}$was suppressed. This reciprocal action on positive and negative 
species implies that these molecules act mainly by reducing the electrostatic potential of the membrane interior with respect to the aqueous phase. Since the modification of ion transport is more prominent at high hydrogen ion concentration (Fig. 6), i.e., when the pesticides exist in aqueous phase as neutral molecules, we assume that the effect originates from the presence of neutral molecules in the membrane. The charge asymmetry of the effect of 2,4-D can be understood in terms of a dipole hypothesis proposed to explain the effect of phloretin (66), cholesterol (61) and salicylamide (70) on ion transport in lipid bilayers. We will show that our results are consistent with the concept of dipole layer at membrane/water interface due to adsorbed neutral molecules of 2,4-D and presumably of other phenoxy acids, IAA and amino acid conjugates.

The changes of voltage dependence of the nonactin- $\mathrm{K}^{+}$conductance in the presence of $2,4-D$ (Fig. $8 b$ ) suggest that the kinetics of ion transport across the membrane interior change. According to the nonactin- $\mathrm{K}^{+}$transport model, the ratio $\mathrm{G}(\mathrm{V}) / \mathrm{G}(0)$ depends on the parameter "A" (Eq. 14) which is a combination of two sets of rate constants and alkali ion concentration, i.e.,

$$
A=2 k_{I S O} / k_{D}+k_{I S O} k_{R}{ }^{c}{ }_{k} / k_{S} k_{D}
$$

There are two types of processes which can limit the flow of potassium ions across the membrane: (1) slow rate of recombination of carriers

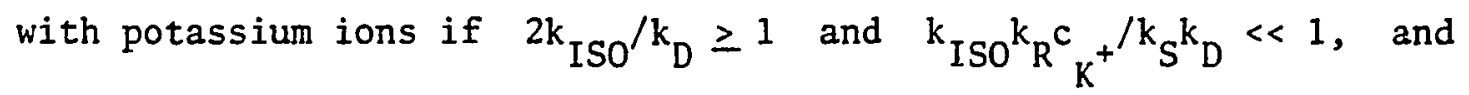
(2) slow rate of back diffusion of unloaded carriers if $\mathrm{k}_{\mathrm{ISO}} \mathrm{k}_{\mathrm{R}}{ }_{\mathrm{K}^{+}}{ }^{\prime}$ $\mathrm{k}_{\mathrm{S}} \mathrm{k}_{\mathrm{D}} \geq 1$ and $2 \mathrm{k}_{I S O} / \mathrm{k}_{\mathrm{D}} \ll 1$. Only the second process depends upon 
the potassium ion concentration, and therefore it is possible to distinguish experimentally between the two types of kinetic limitations developed in the presence of 2,4-D. This was achieved by studying the kinetic parameter $A$ as a function of potassium ion concentration As explained in the methods section, the kinetic parameter A was obtained from the fit of Eq. 13 to the experimental $G(V) / G(0)$ data (Figs. 9b-d) for the $\mathrm{K}^{+}$ion concentration dependence of nonactin- $\mathrm{K}^{+}$ conductance at three different 2,4-D concentrations. The data are shown in Fig. 32. These results clearly indicate that both types of transport 1 imitations are effective in the presence of 2,4-D. At low potassium concentration (between 0.03 and $0.5 \mathrm{M}$ ) the parameter $\mathrm{A}$ is approximately independent of $\mathrm{K}^{+}$concentration and progressively increases with 2,4-D concentration, which means that the rate of complexation on the positively biased membrane side becomes limited by the magnitude of the recombination rate constant $k_{R}$. At higher potassium ion concentration (above $0.5 \mathrm{M}$ ), the value of the parameter $A$ further increases, and thus an additional limiting step becomes significiant. In this case, as follows from the model, the limitation can be attributed to the depletion of unloaded ion carriers at the positively biased membrane surface. We have applied linear regression analysis (Eq. 14) to the experimental dependence of parameter $A$ on the concentration of $\mathrm{K}^{+}$and obtained two sets of combination rate constants: $2 k_{I S O} / k_{D}$ and $k_{I S O} k_{R} / k_{S} k_{D}$. Their values are given in Table 1. Note that both combinations of rate constants change with the concentration of 2,4-D by about the same factor (Fig. 32), which indicates that $2,4-\mathrm{D}$ changes primarily the ratio $\mathrm{k}_{\mathrm{ISO}} / \mathrm{k}_{\mathrm{D}}$ since this 


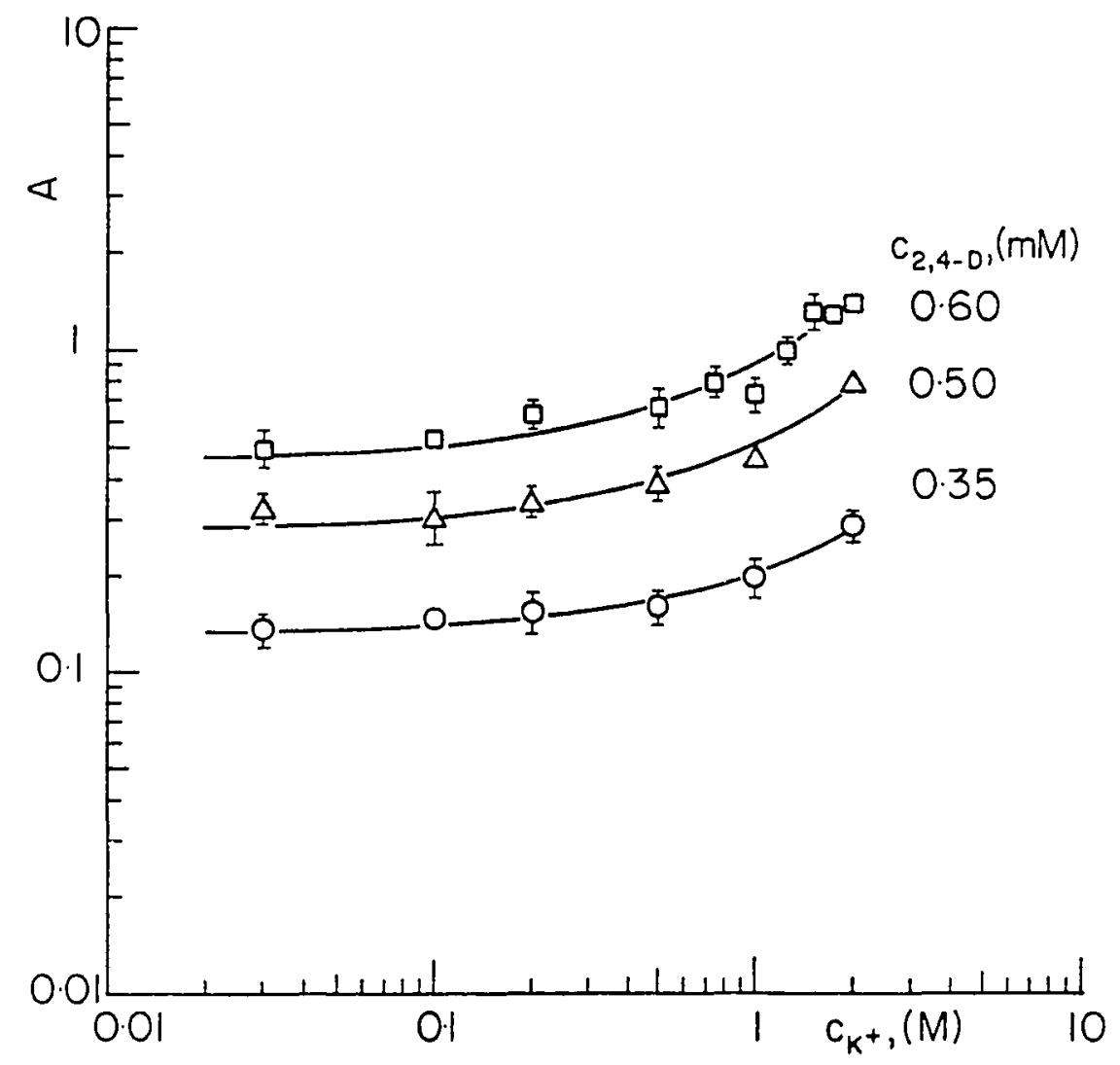

Figure 32. Dependence of kinetic parameter $A$ on the potassium ion concentration for three concentration levels of 2,4-D. The solid curves represent the fit of Eq. 14 for the parameters given in Table 1 . 
ratio is present in both terms of parameter A. The results also suggest that within the above 2,4-D concentration range, the ratio $\mathrm{k}_{\mathrm{R}} / \mathrm{k}_{\mathrm{S}}$ is not very sensitive to $2,4-\mathrm{D}$ concentration (less than factor of 2 change).

According to the adopted model, the conductance of the membrane is also proportional to $\mathrm{k}_{\mathrm{ISO}} / \mathrm{k}_{\mathrm{D}}(\mathrm{Eq} .12)$. Thus the increase of zero voltage membrane conductance and the changes of the voltage dependence of conductance in the presence of 2,4-D originate from the changes of the ratio $\mathrm{k}_{\mathrm{ISO}} / \mathrm{k}_{\mathrm{D}}$.

It is of interest to examine the question of the effect of 2,4-D on the ion-carrier recombinat on process at the membrane surface since it has been found that it can be modified by the presence of membrane additives, such as sterols (62-64). From the model (Eqs. 12-14) and from the numerical values of the rate constant combinations presented in Table 1, it follows that at sufficiently low potassium concentration the second limiting process is inefficient. In that case the second term in the parameter A can be neglected, and the following approximations hold:

$$
\begin{gathered}
\mathrm{A} \approx 2 \mathrm{k}_{\mathrm{ISO}} / \mathrm{k}_{\mathrm{D}} \\
\mathrm{G}(0) \approx\left(\mathrm{e}_{\mathrm{C}^{+}}^{2} / 2 \mathrm{kT}\right) \cdot \mathrm{Ak}_{\mathrm{R}_{\mathrm{s}}} /(\mathrm{I}+\mathrm{A}) .
\end{gathered}
$$

The experiment on the effect of 2,4-D on membrane conductance whose results are shown in Fig. 8 was done at sufficiently low potassium concentration so that the above approximations are applicable. Thus it is possible to evaluate the effect of 2,4-D on the product of 
TABLE I

CHANGE OF KINETIC PARAMETERS OF NONACTIN-K ${ }^{+}$IN PC-CHOL MEMBRANES WITH 2,4-D CONCENTRATION AS OBTAINED FROM THE POTASSIUM CONCENTRATION DEPENDENCE (IONIC STRENGTH $=2 \mathrm{M}$ )

\begin{tabular}{llcc}
$\begin{array}{l}c_{2,4-D} \\
(\mathrm{mM})\end{array}$ & ${ }^{2 k_{I S O} / k_{D}}$ & $\begin{array}{c}k_{I S O} k_{R} / k_{D} k_{S} \\
\left(M^{-1}\right)\end{array}$ & $\begin{array}{c}k_{R} / k_{S} \\
\left(M^{-1}\right)\end{array}$ \\
\hline 0.35 & $0.13 \pm 0.01$ & $0.07 \pm 0.01$ & $1.08 \pm 0.17$ \\
0.50 & $0.28 \pm 0.02$ & $0.24 \pm 0.02$ & $1.71 \pm 0.19$ \\
0.60 & $0.45 \pm 0.05$ & $0.46 \pm 0.05$ & $2.04 \pm 0.32$ \\
\hline
\end{tabular}

the recombination rate constant and the surface density of nonactin $k_{R} N_{S}$, using the data on zero voltage conductance and the parameter $A$, in combination with Eqs. $28 \mathrm{a}$ and $28 \mathrm{~b}$. In Table $2 \mathrm{G}(0), 2 \mathrm{k}_{\mathrm{ISO}} / \mathrm{k}_{\mathrm{D}}{ }^{4}$ and $k_{R} N_{S}$ as a function of 2,4-D concentration were compared. The results suggest that at low 2,4-D concentrations $\left(c_{2,4-D}<0.3 \mathrm{mM}\right)$ the enhancement of potassium ion transport by 2,4-D is primarily due to the increase (100-fold) of the product $k_{R} N_{S}$. Both $k_{R}$ and $N_{S}$ are expected to be sensitive to 2,4-D induced changes of the membrane surface. Another membrane modifier, cholesterol, has been found to increase partition of another ion carrier, valinomycin, into the membrane, and to decrease the recombination rate constant with $\mathrm{Rb}^{+}$ ions (63). Unfortunately, steady state measurements alone do not

${ }^{4}$ The ratios of $k_{I S O} / k_{D}$ obtained from the data in Fig. 8 and these from the potassium concentration dependence as given in Table 1 are different because different ionic strength was used in those experiments. 
TABLE II

EFFECTS OF 2,4-D CONCENTRATION ON KINETIC CHARACTERISTICS OF NONACTIN-K+ TRANSPORT IN PC-CHOL MEMBRANES.

IONIC STRENGTH $=1 \mathrm{M}$

\begin{tabular}{lccc}
$\begin{array}{l}\mathrm{C}_{2,4-\mathrm{D}} \\
(\mathrm{mM})\end{array}$ & $\mathrm{G}(0)$ & $\mathrm{A} \approx 2 \mathrm{k}_{\mathrm{ISO}} / \mathrm{k}_{\mathrm{D}}$ & $\mathrm{k}_{\mathrm{R}} \mathrm{N}$ \\
$(\mathrm{cm} / \mathrm{s})$ \\
\hline 0 & $(0.26 \pm 0.04) \times 10^{-7}$ & $0.01 \pm 0.01$ & $(0.23 \pm 0.12) \times 10^{-7}$ \\
0.05 & $(0.13 \pm 0.03) \times 10^{-6}$ & $0.02 \pm 0.01$ & $(0.67 \pm 0.39) \times 10^{-7}$ \\
0.10 & $(0.45 \pm 0.15) \times 10^{-6}$ & $0.01 \pm 0.01$ & $(0.31 \pm 0.19) \times 10^{-6}$ \\
0.20 & $(0.57 \pm 0.10) \times 10^{-5}$ & $0.02 \pm 0.01$ & $(0.30 \pm 0.12) \times 10^{-5}$ \\
0.30 & $(0.23 \pm 0.06) \times 10^{-4}$ & $0.05 \pm 0.01$ & $(0.44 \pm 0.13) \times 10^{-5}$ \\
0.40 & $(0.57 \pm 0.07) \times 10^{-4}$ & $0.08 \pm 0.01$ & $(0.69 \pm 0.14) \times 10^{-5}$ \\
0.50 & $(0.20 \pm 0.04) \times 10^{-3}$ & $0.14 \pm 0.01$ & $(0.15 \pm 0.03) \times 10^{-4}$ \\
0.60 & $(0.33 \pm 0.04) \times 10^{-3}$ & $0.26 \pm 0.04$ & $(0.14 \pm 0.02) \times 10^{-4}$ \\
0.70 & $(0.55 \pm 0.11) \times 10^{-3}$ & $0.41 \pm 0.07$ & $(0.16 \pm 0.04) \times 10^{-4}$ \\
0.80 & $(0.76 \pm 0.10) \times 10^{-3}$ & $0.68 \pm 0.04$ & $(0.17 \pm 0.02) \times 10^{-4}$ \\
0.90 & $(0.15 \pm 0.02) \times 10^{-2}$ & $0.74 \pm 0.13$ & $(0.30 \pm 0.04) \times 10^{-4}$ \\
1.00 & $(0.21 \pm 0.04) \times 10^{-2}$ & $1.53 \pm 0.28$ & $(0.31 \pm 0.06) \times 10^{-4}$ \\
\hline
\end{tabular}

make it possible to separate the increase of conductance due to the increase of $k_{R}$ from that of $N_{S}$. Also within this 2,4-D concentration range, the ratio of the rate constants $k_{I S O} / k_{D}$ remains small. Its value is about $7 \times 10^{-3}$, which compares favorably with $4 \times 10^{-2}$ for GMO membranes obtained by Hladky $(90,91)$. At high 2,4-D concentrations $\left(c_{2,4-D}>0.3 \mathrm{mM}\right)$ the product $\mathrm{k}_{\mathrm{R}} \mathrm{N}_{\mathrm{S}}$ approaches saturation. However, the increase of membrane conductance in this range is primarily due to the increase of ratio $k_{I S O} / k_{D}$. This ratio of $2,4-D$ 
modified PC-chol membranes can exceed that of nonactin- ${ }^{+}$or trinactin$\mathrm{K}^{+}$for GMO membranes $(90,91)$. Since the difference in conductance as well as in the ratio of $k_{I S O} / k_{D}$ of monoolein and phosholipid membranes can be related to the dipolar potential difference at the membrane boundary $(58,59)$, the increase of $k_{I S O} / k_{D}$ in the presence of 2,4-D supports the hypothesis that the effect of 2,4-D on membranes is also of dipolar nature.

The conductance data obtained on cholesterol free GMO membranes were analyzed in the same manner as those for PC-chol membranes except that $\omega$ was set equal to 0.007 since the thickness of $G M 0 / n-d e c a n e$ membranes estimated from the specific capacitance data in Reference 63 , is about $4.8 \mathrm{~nm}$. In Fig. $332,4-\mathrm{D}$ concentration dependence of parameter A obtained on PC-chol and GMO membranes are compared with the 2,4-D concentration dependence of $\mathrm{TPhAs}^{+}$conductance under identical conditions. It was found that $A_{G M O}>A_{P C-c h o l}$, and the value of parameter $A$ increases exponentially with 2,4-D concentration by about the same factor for both types of membranes. This result contradicts the cholesterol exclusion hypothesis (Section 5e) and strongly suggests that the effect of 2,4-D on cation transport is associated with the change of the ion translocation rate constant, and that the effect is almost independent of the structure of the polar heads. Since at low $\mathrm{K}^{+}$concentration $\mathrm{A} \approx 2 \mathrm{k}_{\mathrm{ISO}} / \mathrm{k}_{\mathrm{D}}$, in the absence of 2,4-D the value of $\mathrm{k}_{I S O} / \mathrm{k}_{\mathrm{D}}$ of $\mathrm{GMO} / \mathrm{n}$-decane membranes is found to be about $0.02 \pm 0.006$. This is smaller but comparable to 0.04 found for nonactin- $\mathrm{K}^{+}$transport in GMO/hexadecane membranes by Hladky $(90,91)$. This result is compatable with the observation that GMO/n-decane membranes are thicker than GMO/hexadecane membranes (63). 


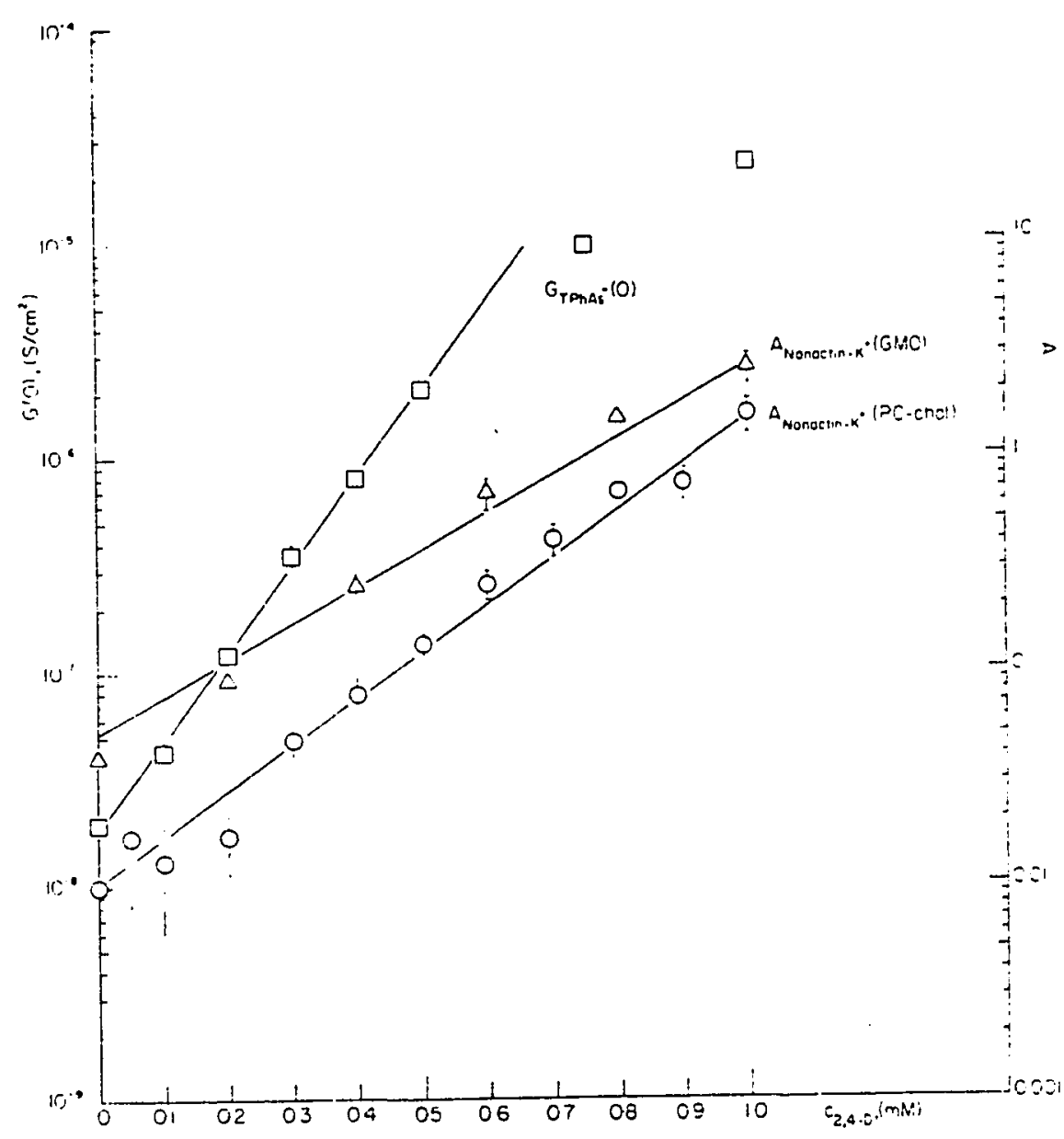

Figure 33. A comparison of 2,4-D concentration dependence of TPhAs ${ }^{+}$conductance with that of kinetic parameter $A \propto k_{I S O} / k_{D}$ for PC-chol and GMO membranes. Experimental conditions: PC-chol membranes: $\quad c_{\text {TPhAs }}{ }^{+}=2 \times 10^{-3} \mathrm{M}$, in $c_{\text {LiCl }}=0.5 \mathrm{M}$. $c_{\text {nonactin }}$ (aqueous sol.) $=1.1 \times 10^{-7} \mathrm{M}, c_{\text {nonactin }}$ (membrane so1.) $=$ $3.2 \times 10^{-5} \mathrm{M}, c_{\mathrm{K}^{+}}=0.06 \mathrm{M}$, ionic strength $(\mathrm{LiCl}+\mathrm{KCl})=1 \mathrm{M}$, $\mathrm{pH}=2$ (buffered). GMO membranes: $c_{\text {nonactin }}$ (aqueous solution) $=$ $1 \times 10^{-9} \mathrm{M}, \mathrm{c}_{\text {nonactin }}$ (membrane solution) $=1.2 \times 10^{-6} \mathrm{M}, \mathrm{c}_{\mathrm{K}^{+}}=$ $0.06 \mathrm{M}$, ionic strength $(\mathrm{LiCl}+\mathrm{KCl})=1 \mathrm{M}, \mathrm{pH}=2$ (buffered). The solid lines represent the linear least square fit of $10 \mathrm{~g}$ $\mathrm{G}_{\mathrm{TPhAs}^{+}}{ }^{(0)}$ vs $\mathrm{c}_{2,4-\mathrm{D}}$ and $10 \mathrm{~g} \mathrm{~A}_{\text {nonactin- }} \mathrm{K}^{+}$vs $c_{2,4-\mathrm{D}^{\circ}}$ 
The results of studies of the effect of ionic strength on membrane conductance due to $\mathrm{TPhAs}^{+}$and nonactin- $\mathrm{K}^{+}$at high concentration of 2,4-D are shown in Fig. 13. In general, the increase of membrane conductance can be attributed to either the increase of the density of membrane permeable ions at the membrane surface and/or to the increase of the ion translocation rate constant. The effect of the ionic strength depicted in Fig. 13a cannot originate from the screening of a possible positive charge at the membrane surface because (a) in the absence of 2,4-D there is no effect (Fig. $13 a)^{5}$, and (b) the conductance increases exponentially with the ionic strength. Actually, as follows from the theory of the diffuse double layer (107), the conductance, assuming that it is controlled by the membrane surface potential due to membrane surface charge, would depend on the ionic strength $I_{S}$ according to

$$
G \propto \exp \left(- \text { constant } / I_{S}^{1 / 2}\right)
$$

This dependence is incompatible with the experimental results.

The changes of current voltage characteristics of nonactin- $\mathrm{K}^{+}$ complex as a function of ionic strength also suggest that the kinetics of ion transport across the membrane interior change. Using the data from Fig. $13 a$ and arguments similar to those employed in the analysis of 2,4-D concentration dependence, the kinetic parameter $A$ is also

${ }^{5}$ Possible positive charge could arise only if lecithin molecules are charged at this $\mathrm{pH}$, because 2,4-D in any form will not be positively charged. $\mathrm{pH}$ of this experiment was kept at 2 . 
found to increase with the ionic strength. The similarity between the ionic strength dependence of the parameter $A_{n o n-K^{+}}$, which is proportional to $\mathrm{k}_{\mathrm{ISO}} / \mathrm{k}_{\mathrm{D}}$, and that of $\mathrm{TPhAs}^{+}$conductance $\mathrm{G}(0)$, which is also proportional to the rate constant of translocation across the membrane (Fig. 34), also supports the hypothesis that 2,4-D increases the probability of translocation of positive ions across the membrane interior.

6c. EFFECT OF 2,4-D ON TPhB- CONDUCTANCE

Unlike the voltage dependence of nonactin- $\mathrm{K}^{+}$conductance in the presence of $2,4-\mathrm{D}$, the changes of the voltage dependence of $\mathrm{TPhB}^{-}$ conductance and the time constant with 2,4-D concentration were very smal1 (Figs. 10 and 11). The solid curves in the Figs, 10 and 11 are the theoretical predictions of the discussed $\mathrm{TPhB}^{-}$simple transport model at 2,4-D concentrations $0,0.1$ and $0.5 \mathrm{mM}$. As can be seen, the model which was first formulated by Andersen and Fuchs (35) can be successfully applied to the membrane perturbed by the presence of 2,4-D.

Comparing these results with those given in Reference 35 , we find that for the untreated membranes, the zero voltage conductance of PC-chol and bacterial phosphatidylethanolamine (BPE) are very similar, whereas the relaxation time constant of PC-chol membranes is greater than that of BPE membranes. For example, for $\mathrm{c}_{\mathrm{TPhB}^{-}}=1 \times 10^{-7} \mathrm{M}$, the zero voltage conductance is about $3 \times 10^{-4} \mathrm{~s} / \mathrm{cm}^{2}$ for PC-chol membranes and $4 \times 10^{-4} \mathrm{~s} / \mathrm{cm}^{2}$ for BPE membranes. The relaxation time constant of PC-chol membranes was found to be $(5-6) \times 10^{-3} \mathrm{~s}$ as compared with $(1-2) \times 10^{-3} \mathrm{~s}$ for BPE membranes $(35,40)$. 


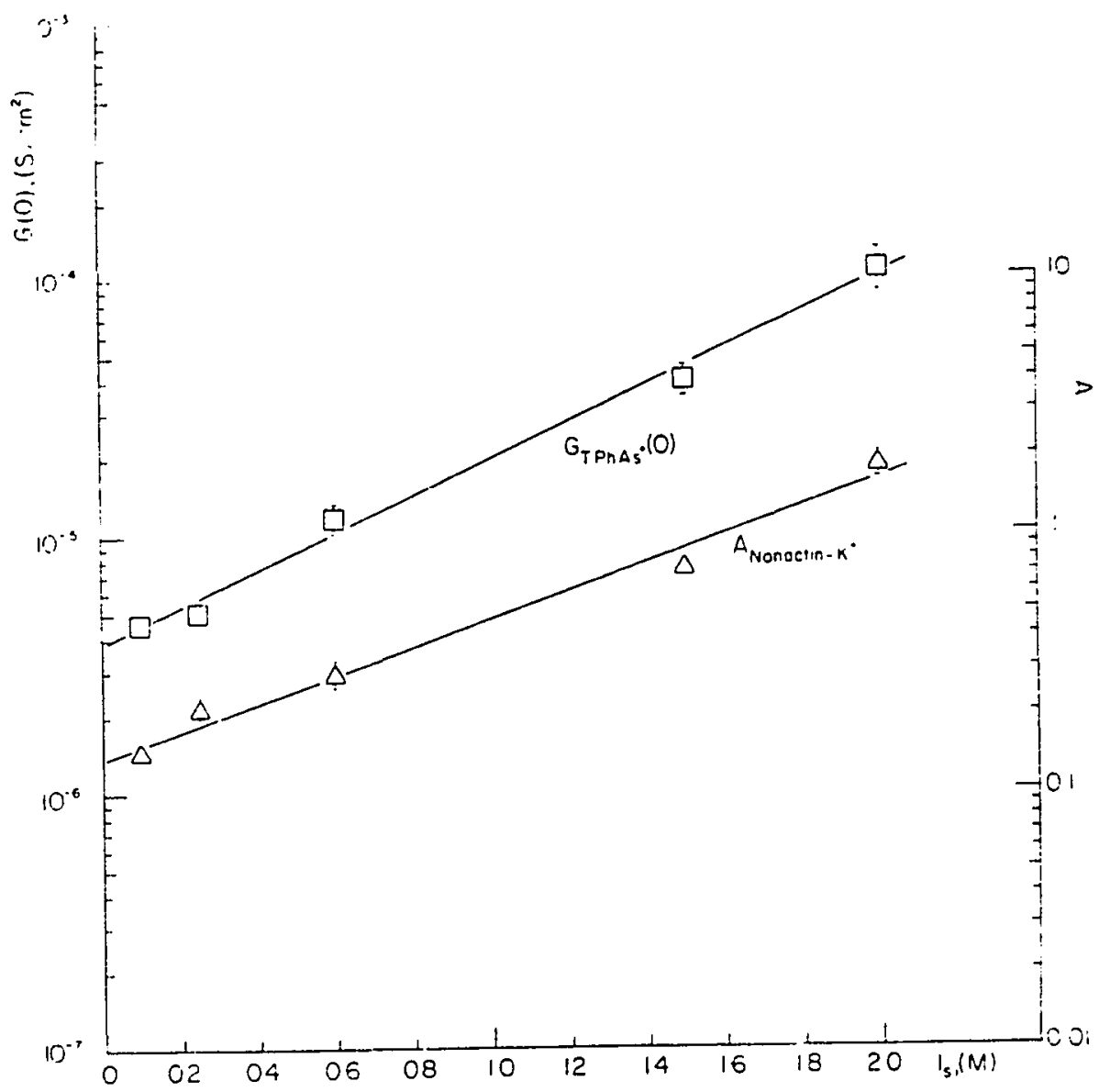

Figure 34. A comparison of ionic strength dependence of $\mathrm{TPhAs}^{+}$ conductance with that of parameter $A \propto k_{I S O} / k_{D}$ at fixed 2,4-D concentration. Experimental conditions: PC-chol membranes; $c_{2,4-D}=7.5 \times 10^{-4} \mathrm{M}, c_{\text {nonactin }}$ (aqueous sol.) $=4.5 \times 10^{-8} \mathrm{M}$; $\mathrm{c}_{\mathrm{TPhAS}^{+}}=2 \times 10^{-3} \mathrm{M} ; \quad c_{\mathrm{K}^{+}}=0.1 \mathrm{M}, \mathrm{pH}=2$ (buffered). The solid lines represent the linear least square fit of $\log \mathrm{G}_{\mathrm{TPhAs}^{+}}(0)$ vs ionic strength and $\log A_{\text {nonactin }-K^{+}} v s$ ionic strength. 
From the least square fit of Eqs. 20,22 , and 25 to the experimental data, the value of $\beta$, which is the fraction of voltage applied between the adsoprtion planes was obtained. Parameter $\beta$ as a function of 2,4-D concentration is shown in Fig. 35. The values of parameter $\beta$ for switch-ON and switch-OFF transients $\left(\beta^{O N}\right.$ and $\left.\beta^{O F F}\right)$ was found to be different. Furthermore, $\beta^{\mathrm{ON}}$ monotonically increased with 2,4-D, whereas $\beta^{\text {OFF }}$ remained unchanged. At high 2,4-D concentrantion ( $5 \times 10^{-4} \mathrm{M}$ and above), the value of $B$ cannot be accurately determined because of the poor signal to noise ratio of the transient currents, especially at low bias voltages. The dependence of $\beta$ on 2,4-D concentration can be understood in terms of the changes of adsorption of $\mathrm{TPhB}^{-}$.

The net charge per unit membrane area, translocated across the membrane as a function of applied potential difference and 2,4-D concentration, is given in Fig. 12. For the purpose of illustration of the model, the curves of this plot represent the voltage dependence of transferred charge, as given by Eq. 24. The limiting value of $Q(V)$ at high voltages is equal to the surface density due to the adsorbed $\mathrm{TPhB}^{-}$ions, $Q_{\text {ads }}$. The results indicate that 2,4-D inhibits adsorption of $\mathrm{TPhB}^{-}$since $Q_{\text {ads }}$ decreases with the increasing 2,4-D concentration. In the absence of $2,4-D$, the surface charge density of PC-chol membranes in the presence of $1 \times 10^{-7} \mathrm{M} \mathrm{TPhB}^{-}$is about $1 \times 10^{-7}$ coulomb $/ \mathrm{cm}^{2}$, which is comparable to that found for BPE membranes $(35,40)$. The values of parameter $B$ for PC-chol and BPE membranes are also similar: $\beta^{O N}(P C-c h o 1) \approx 0.6$ as compared to $B(B P E)=0.71(40)$ for comparable $\mathrm{TPhB}^{-}$concentrations $\left(1-3 \times 10^{-7} \mathrm{M}\right)$. The increase of 


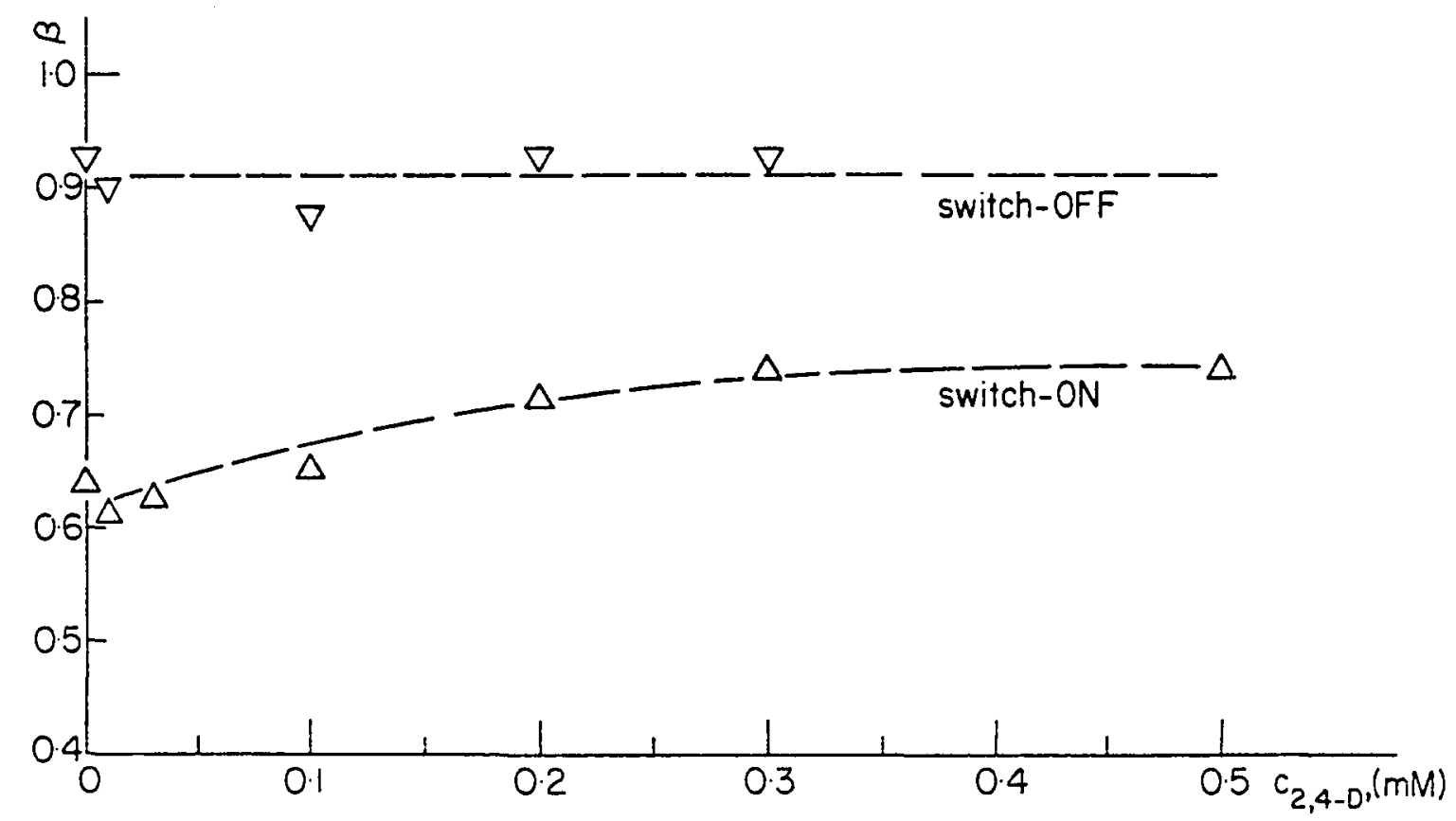

Figure 35. Dependence of parameter $\beta$ on 2,4-D concentration for the switch-ON and switch-OFF transient processes. The value of $B$ has been obtained from the best fit of the model equations to the voltage dependence of the initial membrane conductance and the relaxation time constant. 
$\beta^{O N}$ in the presence of $2,4-D$ can be interpreted as a consequence of inhibition of $\mathrm{TPhB}^{-}$adsorption by 2,4-D. In terms of the "three capacitor model," as demonstrated by Andersen et al. (40), the effective potential difference driving the ion diffusion across the membrane decreases with the increasing surface charge density of the membrane permeable ions. The observed increase of $\beta^{O N}$ and the associated decrease of the surface charge density $Q_{a d s}$ with the increasing concentration of 2,4-D (Fig. 12) are consistent with the conclusions derived from the three capacitor model $(40)$. The observed monotonic change of $\beta^{\text {ON }}$ with the decrease of $\mathrm{TPhB}^{-}$surface charge density indicates that the assumption of negligible potential difference between the $\mathrm{TPhB}^{-}$adsorption plane and the aqueous medium (as compared to $\mathrm{kT} / \mathrm{e}$ ) implied in the adopted model (35), is not strictly satisfied. From this stardpoint it would be desirable to do a similar study of $\mathrm{TPhB}^{-}$concentration well below $10^{-7} \mathrm{M}$. This condition however would limit the 2,4-D concentration range because of the smaller signal to noise ratio of the transient currents. We consider $\beta^{\mathrm{OFF}}$ to be a better parameter for $\mathrm{TPhB}^{-}$transport in PC-chol membranes. The value of $\beta^{\text {OFF }}(\mathrm{PC}-\mathrm{chol})=0.91$ obtained here is close to $B(B P E)=0.86$ determined at low $\mathrm{TPhB}^{-}$concentration $\left(c_{\mathrm{TPhB}^{-}}=1 \times 10^{-8} \mathrm{M}(40)\right)$ and to $B$ (diolelphosphatidyl ethanolamine) $=0.92$ (35). The origin of the difference between $\beta^{\text {ON }}$ and $\beta^{\text {OFF }}$ is not understood.

At higher 2,4-D concentration, the redistribution time of $\mathrm{TPhB}^{-}$ ions in the membrane increases (Fig. 11). There is a possibility that during the measurement of the relaxation current, the condition of isolation of $\mathrm{TPhB}^{-}$ions trapped in the membrane from the aqueous solution, as implied in the model, is violated, because of the outflow of 
ions from the positively biased membrane side and inflow of ions to the negatively biased side. This possibility was checked by comparing the amount of charge transported during the switch-ON and switch-OFF transient conduction. It was found that the exchange of $\mathrm{TPhB}^{-}$between the membrane and the aqueous solution was at the most $20 \%$, and thus the assumption of complete trapping of $\mathrm{TPhB}^{-}$ions in the membrane remains approximately valid.

The 2,4-D induced changes of membrane conductance characteristics, as illustrated in Figs. 10,11, and 12, provide more detailed information than the steady-state studies with positive ions because of the possibility of determining separately the effect of 2,4-D on the density of the adsorbed $\mathrm{TPhB}^{-}$ions and on their translocation across the membrane. The decrease of membrane permeability to $\mathrm{TPhB}^{-}$ions as evidenced by the decrease of membrane conductance is in part due to the smaller adsorption coefficient of $\mathrm{TPhB}^{-}$ions at the membrane surface, and in part due to the change of the kinetics of ion translocation. The kinetic aspect of blocking of the ion transport must be the dominating one, as can be clearly seen in Fig. 36 where the decrease of membrane conductance has been compared with the changes of $\mathrm{TPhB}^{-}$ adsorption. For example, an increase of 2,4-D concentration from 0 to $4.5 \times 10^{-4} \mathrm{M}$ corresponds to a decrease of the density of surface charge due to adsorbed $\mathrm{TPhB}^{-}$by a factor of about 3 , whereas the conductance decreases about 38 -fold.

The changes of the kinetics of $\mathrm{TPhB}^{-}$transport are directly reflected in the changes of the relaxation time constant. A semilog plot of the dependence of the relaxation time constant on 2,4-D 
concentration indicates that the redistribution time of $\mathrm{TPhB}^{-}$ions in the membrane exponentially increases in the presence of 2,4-D (Fig. 37).

6d. 2,4-D INDUCED CHANGES OF ELECTRIC POTENTIAL OF MEMBRANE INTERIOR

The plots of the dependence of membrane conductance, density of surface charge due to adsorbed $\mathrm{TPhB}^{-}$ions, the relaxation time constant versus 2,4-D concentration (Figs. 36 and 37) and the plots of TPhAs ${ }^{+}$ conductance, parameter A of nonactin- $\mathrm{K}^{+}$complex for $\mathrm{PC}-\mathrm{chol}$ and GMO membranes versus 2,4-D concentration (Fig. 33) suggest that these quantities change exponentially with $2,4-D$ concentration. Since the experimental data on membrane conductance (Fig. 5) indicate that the effect of 2,4-D is asymmetric with respect to the sign of the electric charge of the transported ion, it is reasonable to assume that 2,4-D changes the electrostatic potential of the membrane interior.

Using the changes of $\mathrm{TPhAs}^{+}$conductance, $\mathrm{TPhB}^{-}$conductance, and the changes of parameter $A$ of the nonactin $-\mathrm{K}^{+}$complex, and the relaxation time constant for $\mathrm{TPhB}^{-}$, one can estimate the changes of the electric potential of the membrane interior induced by $2,4-D$. The electric potential difference between the central region of the membrane core and the bulk aqueous solution $\psi$ has two components (see inset in Fig. 38): the potential difference between the center of the membrane and the adsorption-reaction plane $\phi$ and the potential difference between the adsorption-reaction plane and the aqueous solution $\theta$, i.e.,

$$
\psi=\theta+\Phi
$$




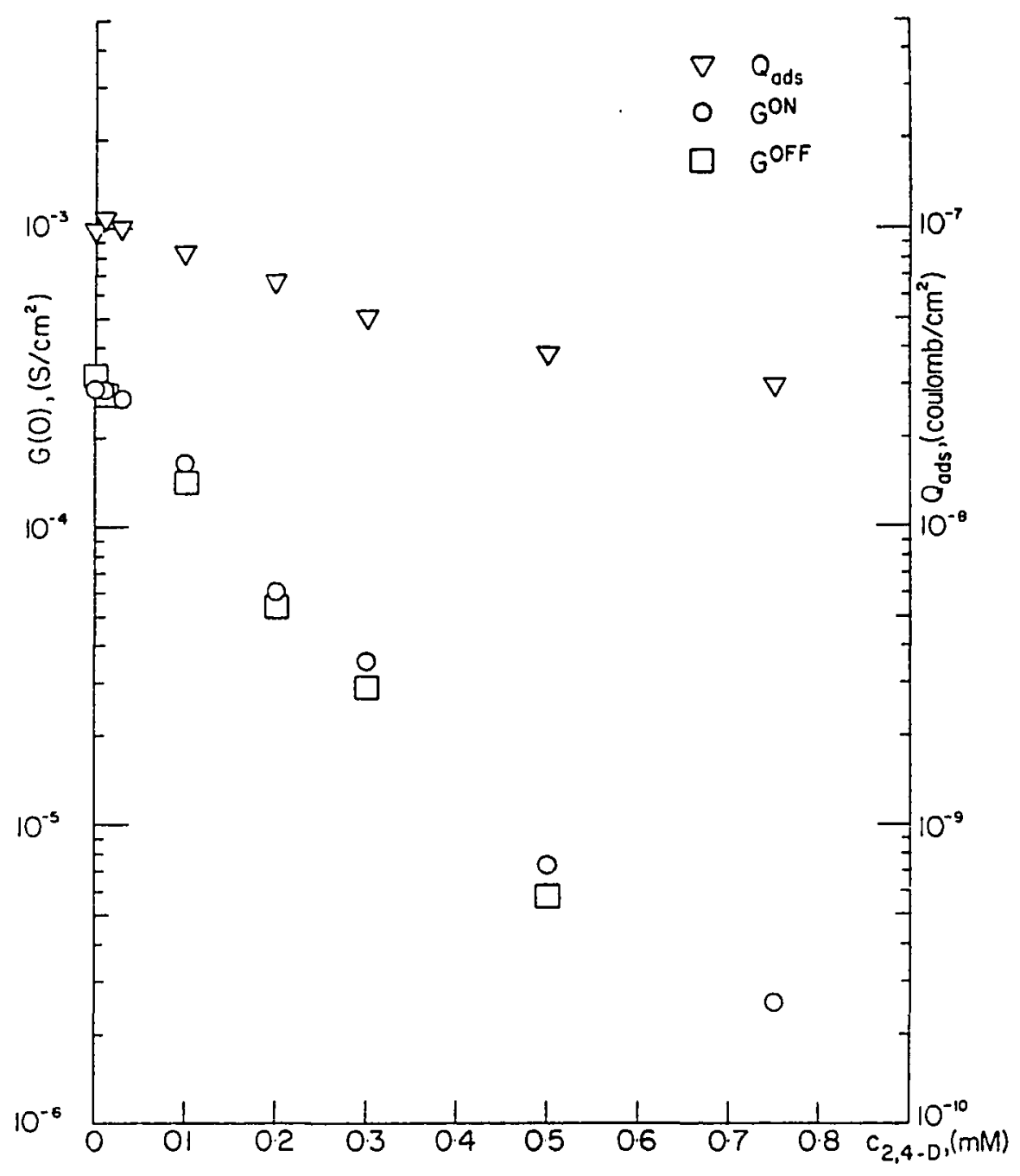

Figure 36. A comparison of 2,4-D concentration dependence of switch-ON and switch-OFF $\mathrm{TPhB}^{-}$membrane conductance with the 2,4-D concentration dependence of the density of surface charge due to $\mathrm{TPhB}^{-}$ions adsorbed at the membrane surface. 


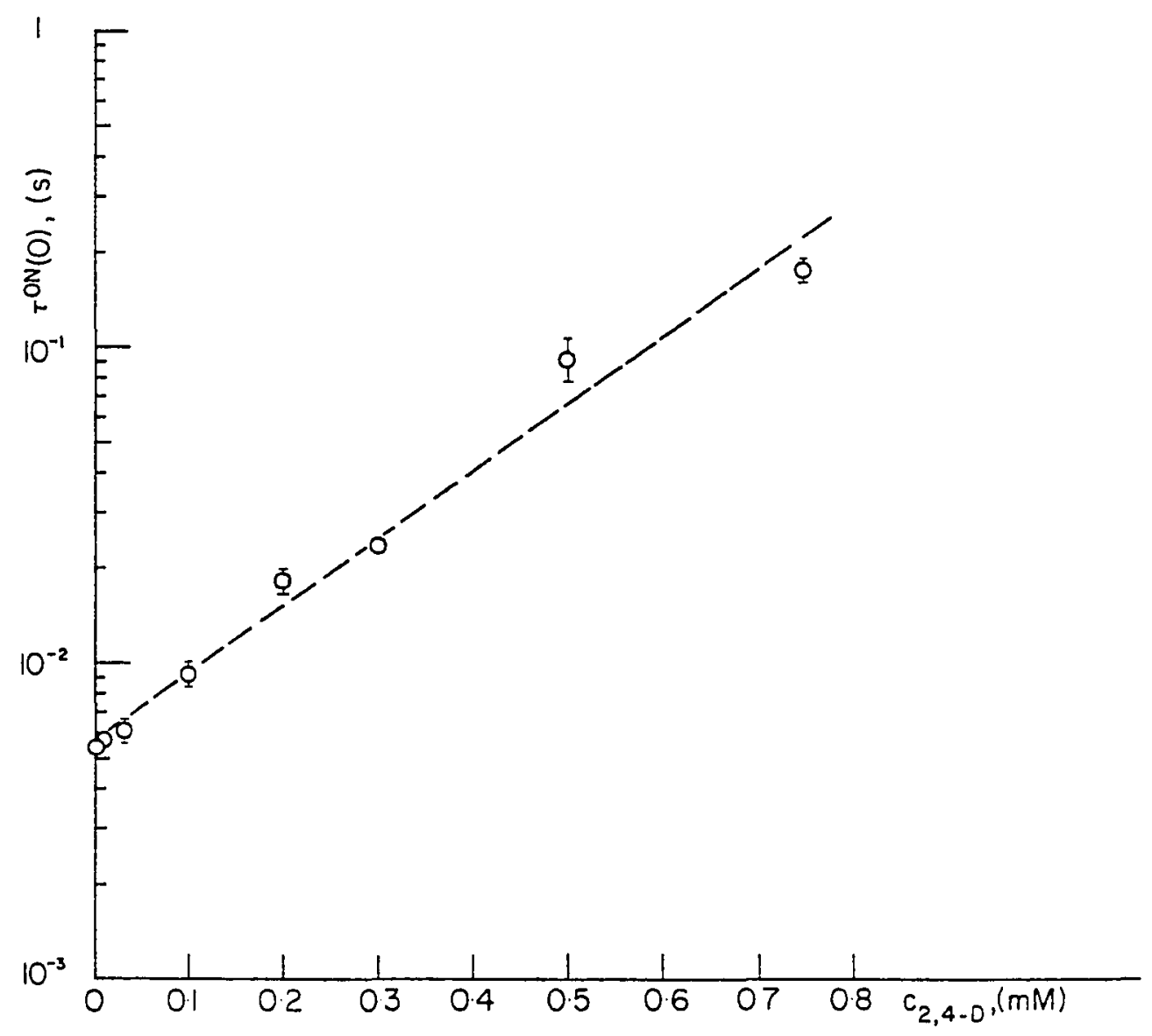

Figure 37. A plot of the dependence of zero voltage relaxation time constant for $\mathrm{TPhB}^{-}$on 2,4-D concentration. The broken line represent the linear least square fit of $\log \tau$ vs $c_{2,4-D^{\circ}}$ 
The changes of $\mathrm{TPhAs}^{+}$and $\mathrm{TPhB}^{-}$conductance depend on both the changes of the density of adsorbed ions, which can be accounted by $\Delta \theta$ as well as on the changes of the translocation rate constant, which can be attributed to $\Delta \phi$. Within this framework, the zero voltage conductance of 2,4-D modified membranes can be related to that of untreated (reference) membrane (superscript ref), by the Boltzmann factor:

$$
\mathrm{G}_{\mathrm{TPhAs}^{+}}(0)=\mathrm{G}_{\mathrm{TPhAs}}^{\mathrm{ref}}(0) \cdot \exp \left(\mathrm{b}_{\mathrm{G}}^{\prime} \mathrm{c}_{2,4-\mathrm{D}}\right)=\mathrm{G}_{\mathrm{TPhAs}}^{\mathrm{ref}}(0) \cdot \exp (-e \Delta \psi / \mathrm{kT})
$$

and

$$
G_{T P h B^{-}}(0)=G_{T P h B^{-}}^{\text {ref }}(0) \cdot \exp \left(b_{G}^{\prime \prime} c_{2,4-D^{-}}\right)=G_{T P B^{-}}^{\text {ref }}(0) \cdot \exp (\operatorname{es\psi /kT})
$$

Thus from the $\mathrm{TPhAs}^{+}$and $\mathrm{TPhB}^{-}$conductance data one can estimate the changes of the height of the potential energy barrier between the center of the meinbrane and the aqueous solution. The changes of the TPhAs ${ }^{+}$ and $\mathrm{TPhB}^{-}$conductance are a measure of the ion partition coefficient and the translocation rate constant.

For the nonactin-K ${ }^{+}$transport, all the previous evidence suggests that the changes of parameter $A$ are dominated by the changes of translocation rate constant $k_{\text {ISO }}$ since the translocation rate constant is associated with the height of the membrane potential energy barrier with respect to the ion adsorption-reaction plane, the changes in $A_{\text {non- }} \mathrm{K}^{+}$reflecting the changes of the potential difference between the center of the membrane and the reaction plane, $\Delta \phi$,

$$
A_{\text {non-K }} \approx A_{\text {non }-K^{+}}^{r e f} \cdot \exp (-e \Delta \phi / k T)
$$


Similarly, the change in the redistribution time of $\mathrm{TPhB}^{-}$ions across the membrane can be related to the change of the ion potential energy difference between the central membrane plane and the adsorption plane $e \Delta \phi$

$$
\tau(0)=\tau^{\text {ref }}(0) \cdot \exp \left(b_{\tau} c_{2,4-D}\right)=\tau^{\text {ref }}(0) / \exp (e \Delta \phi / k T)
$$

Finally, the change of the density of adsorbed $\mathrm{TPhB}^{-}$ions depends on the depth of the ion potential energy well at the adsorption plane e $\Delta \theta$,

$$
Q_{\text {ads }}=Q_{a d s}^{\text {ref }} \cdot \exp \left(b_{Q} c_{2,4-D}\right)=Q_{a d s}^{\text {ref }} \cdot \exp (e \Delta \theta / k T)
$$

Figure 38 illustrates the changes of the potential differences $\Delta \psi$ and $\Delta \phi$ as a function of 2,4-D concentration obtained from the fit of equations 30 and 32 to the $\mathrm{TPhAs}^{+}$conductance data and the parameter $\mathrm{A}$ of nonactin- $\mathrm{K}^{+}$complex in Fig. 33. These potential difference changes are approximately proportional to the concentration of $2,4-D$, and at high 2,4-D concentration they correspond to several $k T$. It is interesting to note that the potential difference change $\Delta \phi$ in both PC-chol and GMO membranes are almost identical.

The changes of electric potential difference with 2,4-D concentration, as determined from the changes of the three independently measured quantities, $\mathrm{TPhB}^{-}$conductance, relaxation time constant and surface charge density due to adsorbed $\mathrm{TPhB}^{-}$ions, are shown in Fig. 39 . Thus in the presence of $2,4-D$, both the potential difference between $\mathrm{TPhB}^{-}$adsorption plane and the aqueous medium, $\Delta \theta$, and that between the membrane interior and the adsorption plane $\Delta \phi$, becomes more negative. Also, one should note that the changes in the potential 


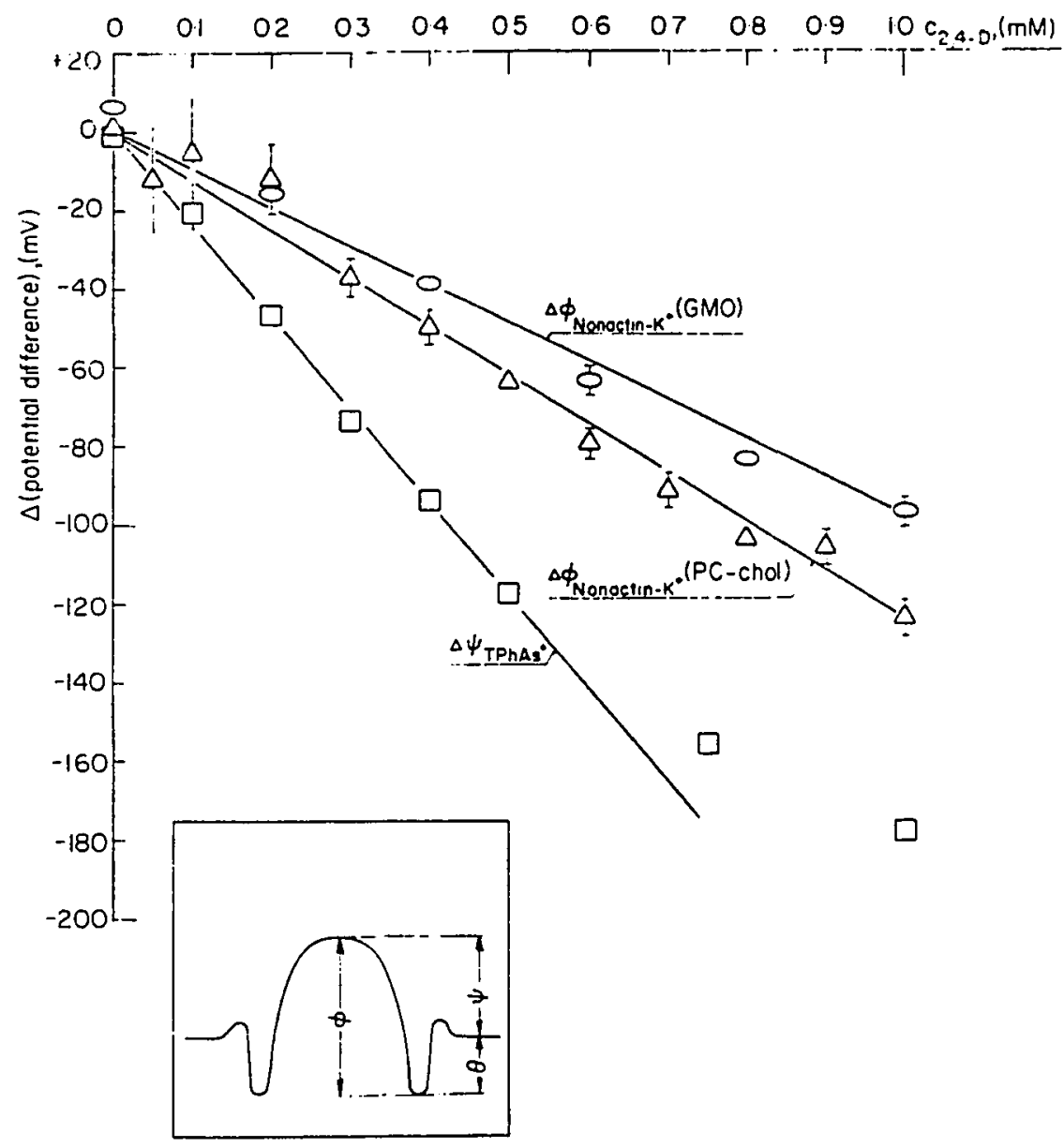

Figure 38. Change of electric potential difference between membrane interior and the aqueous solution $\left(\Delta \psi_{\mathrm{TPhAs}^{+}}\right)$and between the membrane interior and the reaction plane ( $\Delta \phi_{\text {non }-K^{+}}, P C-c h o l$ and GMO membranes) as a function of 2,4-D concentration. The potential difference changes have been estimated from the changes of membrane conductance $\left(\triangle \psi_{\mathrm{TPhAs}^{+}}\right)$and from the changes of the kinetic parameter $A\left(\Delta \phi_{\text {non-K }}{ }^{+}\right)$given in Fig. 34 . The solid lines represent the linear least square fit. 
between the central plane of the membrane and the $\mathrm{TPhB}^{-}$adsorption plane, $\Delta \phi$, are considerably greater than those between the adsorption plane and the aqueous solution $\Delta \theta$.

It is of interest to compare $\Delta \psi$ deduced from the membrane conductance changes with the sum $\Delta \theta+\Delta \phi$ obtained from the separate measurements of the surface charge density $(\Delta \theta)$, and the relaxation time constant $(\Delta \phi)$ since in terms of the adopted barrier model, the barrier height $e \psi=e \theta+e \phi$. If the transport model takes properly into account the changes induced by the presence of $2,4-D$, it is to be expected that $\Delta \psi=\Delta \theta+\Delta \phi$. As follows from the comparison in Fig. 39, this expectation is fulfilled; $\Delta \psi$ (open symbols) agrees closely with the sum $\Delta \theta+\Delta \phi$ (filled symbols).

Finally, the results obtained on positively charged probes can be compared with those derived from $\mathrm{TPhB}^{-}$data. The net changes of the potential difference between the membrane interior and the aqueous solution $\Delta \psi$, obtained from the $\mathrm{TPhAs}^{+}$and $\mathrm{TPhB}^{-}$conductance data are compared in Fig. 40. The 2,4-D-induced electric potential difference, as determined by positive ions is by about $30-40 \%$ greater than that derived from the negative ions. For reasons which are not understood, the changes of conductance due to various membrane modifiers as detected by positively charged probes are often greater than those with negative probes $(40,65)$. In the case of phloretin this discrepancy has been associated with the presence of cholesterol in the membrane (66). In the present work we have noted that $\Delta \theta$, $\Delta \phi$ and $\Delta \psi$, determined from independent measurements, are self consistent in that $\Delta \psi=\Delta \theta+\Delta \phi$. Changes of $\mathrm{TPhB}^{-}$conductance can be accounted for by the changes of $\mathrm{TPhB}^{-}$adsorption and the kinetics 


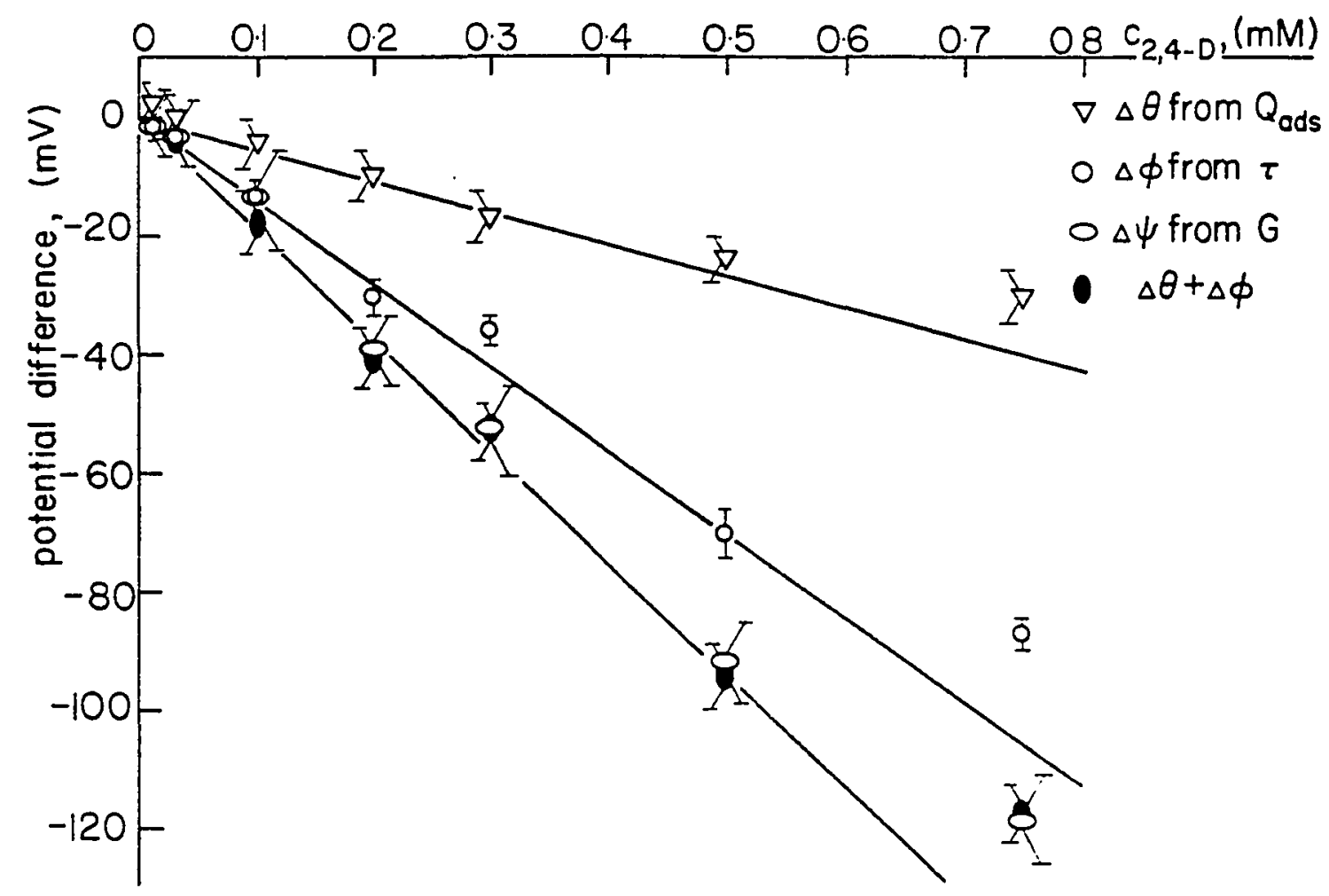

Figure 39. Changes of electric potential differences as a function of aqueous 2,4-D concentration determined from the studies of relaxation of $\mathrm{TPhB}$ - conduction current in $\mathrm{PC}$-chol membranes. $\Delta \theta$ is the change of the potential difference between the $\mathrm{TPhB}^{-}$adsorption plane and the aqueous solution, $\Delta \phi$ is the change of the potential difference between the central membrane plane and the adsorption plane. $\Delta \psi$ is the change of the potential difference between the central membrane plane and the aqueous solution. Solid lines represent the linear least square fit. 


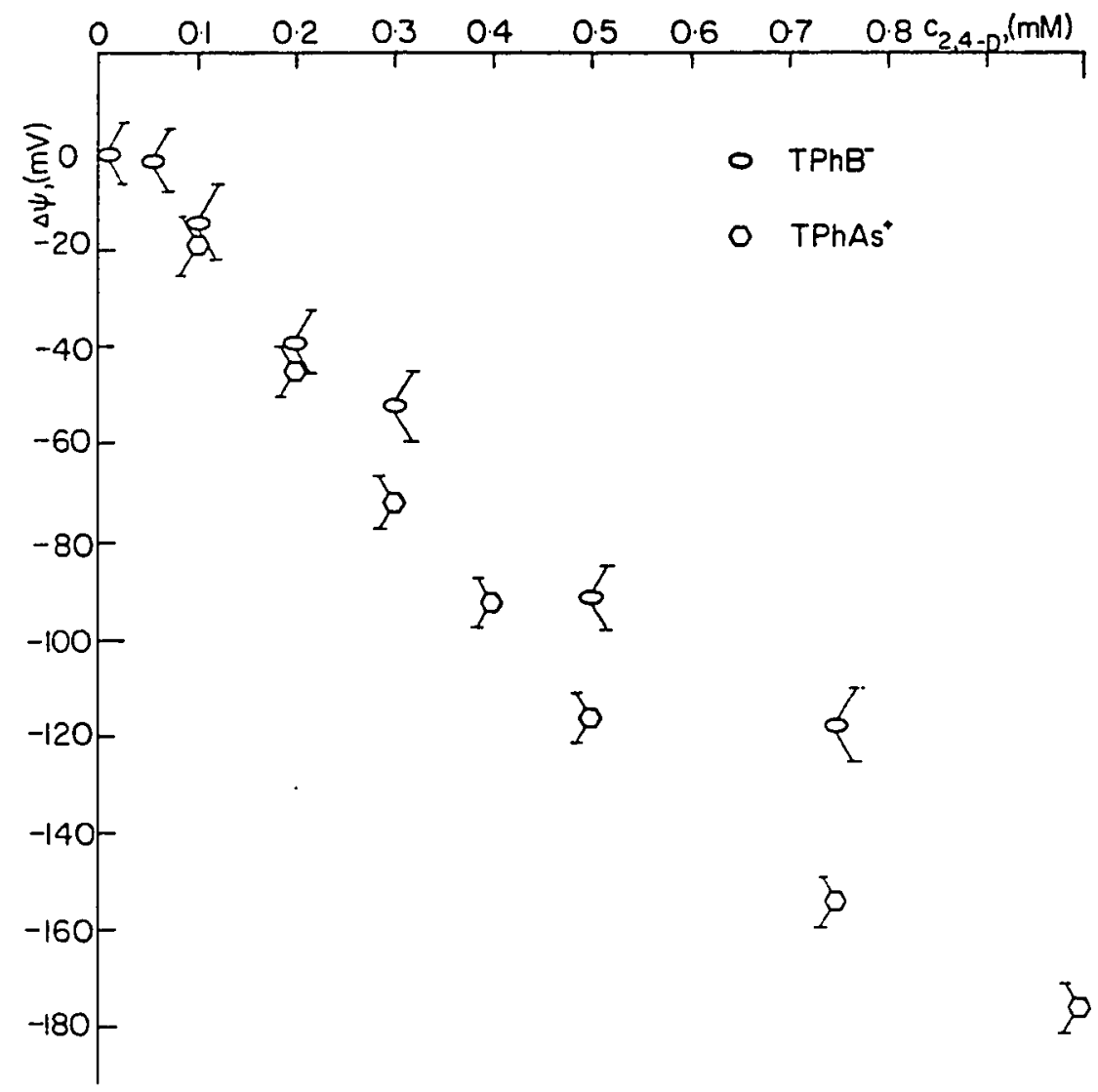

Figure 40. A comparison of the changes of the electric potential difference between the central plane of the membrane and the aqueous solution as determined from the changes of membrane conductance due to $\mathrm{TPhAs}^{+}$and $\mathrm{TPhB}^{-}$ ions. 
of translocation. It is possible that in addition to electrostatic effects as discussed above, 2,4-D facilitates adsorption of positively charged ions at the interface, which would account for the greater enhancement of cationic conductance as compared with the anionic conductance. It is interesting to note that the changes of the potential difference between the membrane interior and the adsorption-reaction plane, as determined from the changes of kinetic parameter $A$ of nonactin- $\mathrm{K}^{+}$transport and from the changes of the relaxation time constant of $\mathrm{TPhB}^{-}$conductance, are similar (Fig. 41). This experimental result suggests the following: (1) Since both the increase of nonactin $-\mathrm{K}^{+}$translocation rate constant and the increase of the $\mathrm{TPhB}^{-}$ relaxation time constant can be accounted for by the change of the height of the ion potential energy barrier, and since the changes as a function of 2,4-D concentration are about the same (Fig. 41), membrane geometry and other properties of membrane interior remain approximately same. This in turn implies that the diffusion coefficient of the membrane permeable ions is not significantly affected by the presence of 2,4-D. (2) The location of the layer of oriented 2,4-D molecules relative to the adsorption plane of $\mathrm{TPhB}^{-}$and the recombination plane of nonactin with $\mathrm{K}^{+}$ions is about the same.

In terms of the adopted hypothesis, the decrease of the electric potential of the membrane interior originates from a layer of 2,4-D molecules adsorbed within the membrane interfacial region. In order to account for the changes of electric potential of the membrane interior, the molecular dipole moments have to be oriented toward the aqueous solution, as depicted in Fig. 42. By treating the dipole layer as two 


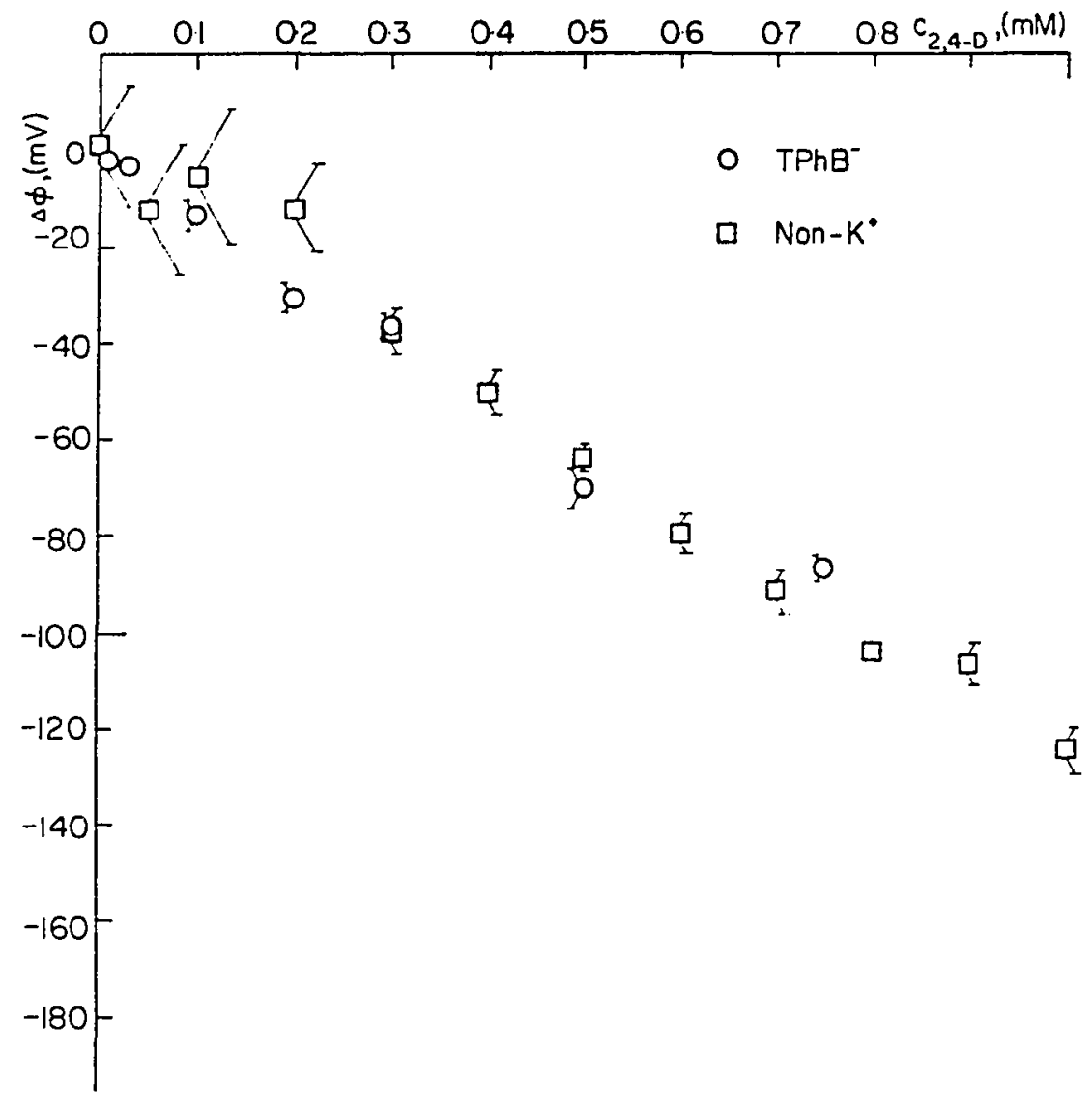

Figure 41. A comparison of the changes of the electrical potential difference between the central plane of the membrane and the adsorption-reaction plane as determined from the changes of kinetics of nonactin- $\mathrm{K}^{+}$transport, $\Delta \phi$ (nonactin- $\mathrm{K}^{+}$), and the relaxation time constant of $\mathrm{TPhB}^{-}$transport, $\Delta \phi\left(\mathrm{TPhB}^{-}\right)$. 


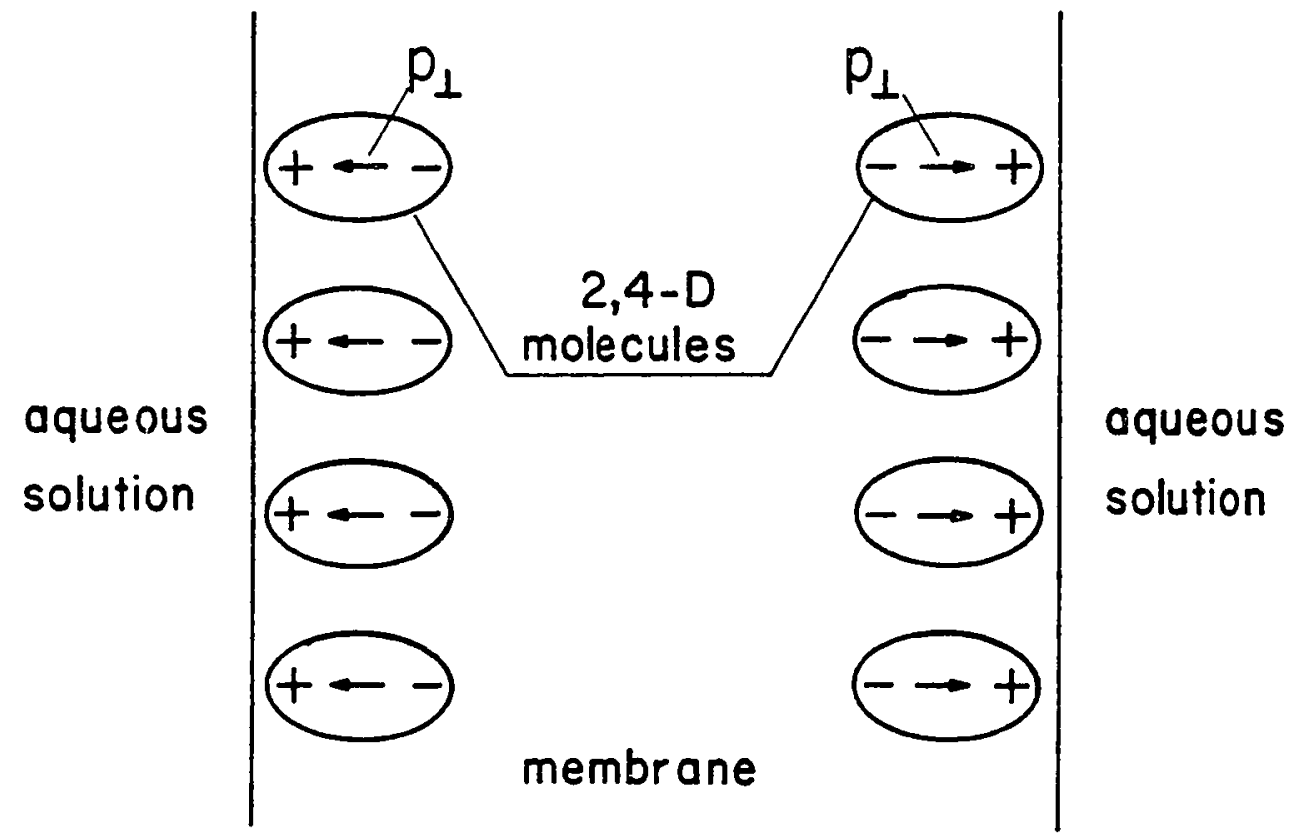

Figure 42. A diagram depicting the dipole hypothesis used to interpret the effect of 2,4-D on ion transport. The neutral 2,4-D molecules are assumed to be adsorbed in the membrane and oriented toward the aqueous solution. This results in lower potential energy of positive ions and higher potential energy of negative ions in the membrane interior, which causes changes in membrane permeability in opposite direction for ions having opposite electric charge. 
sheets of electric charge, the potential difference across the layer is equal to

$$
\mathrm{V}_{\mathrm{dip}}=\mathrm{N}_{2,4-\mathrm{D}} \cdot \frac{\mathrm{p}_{\perp}}{\varepsilon \varepsilon_{0}}=\mathrm{K} \mathrm{c}_{2,4-\mathrm{D}} \cdot \underline{\mathrm{p}}_{\perp} / \varepsilon \varepsilon_{0},
$$

where $\mathrm{N}_{2,4-D}$ is the surface density of oriented 2,4-D molecules, $p_{\perp}$ the component of 2,4-D dipole moment normal to the surface, $K$ the partition coefficient of 2,4-D between the membrane surface and the aqueous solution, and $\varepsilon \varepsilon_{0}$ the permittivity. Equating $\mathrm{V}_{\mathrm{dip}}$ to the potential difference $\Delta \psi$ or $\Delta \phi$ and $p_{1}$ to the dipole moment of 2,4-D molecule (3.33 debye (108)), one can estimate $\mathrm{N}_{2,4-\mathrm{D}} / \varepsilon$. For an electric potential change of $100 \mathrm{mV}, N_{2,4-D} / \varepsilon=7.97 \mathrm{x}$ $10^{16} / \mathrm{m}^{2}$, which for effective dielectric constant of the boundary region $\varepsilon=10$ corresponds to separation between 2,4-D molecules of $1.1 \mathrm{~nm}$.

The increase of both the TPhAs ${ }^{+}$conductance and the kinetic parameter $\mathrm{A}_{\text {non-K+ }}$ with the ionic strength can be also attributed to the increase of the ion translocation rate constant. Since the effect of ionic strength is qualitatively similar to that of 2,4-D concentration (compare Figs. 8 and 13), the partition coefficient of 2,4-D between the membrane interface and the aqueous solution is assumed to increase with the ionic strength. The effect of ionic strength can be qualitatively understood in the following way: as the concentration of electrolyte is increased, the concentration of free water molecules available for the solvation of 2,4-D decreases which results in relatively lower energy state of $2,4-D$ in the membrane with respect to that in the aqueous solution. It is reasonable to assume that the 
distribution coefficient of 2,4-D between the membrane and the aqueous medium has two components as follows:

$$
K=K_{0}+K_{1} I_{s}
$$

$\mathrm{K}_{0}$ is the distribution coefficient between the membrane surface and water, and while $k_{1}$ accounts for "salting out" effect of the electrolyte. Is represents the ionic strength. It then follows that the dependence of $\mathrm{TPhAs}^{+}$conductance on 2,4-D concentration and the ionic strength is given by

$$
G_{T_{P h A s}}(0)=G_{T P h A s^{+}}^{\text {ref }}(0) \cdot \exp \left[\left(b_{0}^{G}+b_{1}^{G_{I}}\right)\left(c_{2,4-D}-c_{2,4-D}^{\text {ref }}\right)\right] .
$$

Similarly, the kinetic parameter A of nonactin- $\mathrm{K}^{+}$transport is given by

$$
A_{\text {non }-K^{+}}=A_{\text {non }-K^{+}}^{\text {ref }} \cdot \exp \left[\left(b_{0}^{A}+b_{1}^{A} I_{s}\right)\left(c_{2,4-D}-c_{2,4-D}^{\text {ref }}\right)\right]
$$

These two equations describe both the effect of 2,4-D and ionic strength. In the framework of the dipole hypothesis, the term $b_{0}$ and $b_{1} I_{s}$ are proportional to the partition coefficients of 2,4-D, $K_{0}$ and $\mathrm{K}_{1} \mathrm{I}_{\mathrm{s}}$, the fraction of the dipolar potential difference that affects the transport process in question, and to the other characteristics of the dipolar layer such as effective dielectric constant of the interface.

6e. 2,4-D INDUCED CHANGES OF INTERFACIAL ELECTRIC POTENTIAL DIFFERENCE

The results of surface potential measurements provide additional and rather direct support for the dipolar hypothesis of 2,4-D action in 
lipid membranes. Figs. 14, 15, and 16 indicate the following:

(a) 2,4-D decreases the electric potential of the nonpolar medium, (b) this effect is largest at $\mathrm{pH} \quad \mathrm{pK}_{\mathrm{a}}$ of 2,4-D molecules, and (c) the decrease of electric potential of the nonpolar side of the interface is further lowered in the presence of electrolyte. These effects can occur only if neutral 2,4-D molecules are adsorbed at the interface and are oriented so that their dipole moment is directed toward the aqueous medium. However, the quantitative agreement between the changes of electric potential difference between the membrane interior and the adsorption-reaction plane or the aqueous solution, as determined from ionic probes for PC-chol membranes and those measured for PC-chol monolayers is rather poor (compare Figs. 39 and 14). The effect of 2,4-D on potential difference across PC-chol monolayer is weak. The same kind of inconsistency has been observed for the action of phloretin in BPE-chol and PC-chol membranes and in monolayers, and has been associated with the presence of cholesterol in the monolayer (66).

Haydon and Meyers (51) found an excellent agreement between the monolayer and the bilayer potential changes for several ionic and zwitterionic surfactants for cholesterol-free GMO membranes and monolayers. Since 2,4-D is also active in GMO membranes, the action of 2,4-D on GMO monolayers have been studied (Fig. 17). In contrast to PC-chol monolayers (Fig. 14), the electric potential of the hydrocarbon side of the GMO monolayer becomes significantly more negative in the presence of 2,4-D (Fig. 17). The straight line in Fig. 17 indicates the linear relationship between the change of the monolayer surface potential difference $\Delta \psi$ and the concentration of 2,4-D. From the least square fit of $\Delta \psi=b c_{2,4-D}$ to the data, we obtain 
$b_{m}(G M O)=1.4 \times 10^{5} \mathrm{mV} / M$ for GMO monolayers as compared with $b_{m}(P C-$ chol) $=0.2 \times 10^{5} \mathrm{mV} / \mathrm{M}$ (poor correlation) for PC-chol monolayers. The results can be compared with the slope $b_{b}(G M O)=0.99 \times 10^{5} \mathrm{mV} / \mathrm{M}$ of the 2,4-D concentration dependence of the potential difference change $\Delta \phi$ for GMO bilayers obtained from the data in Fig. 34, and with $b_{b}(P C-c h o l)=1.2 \times 10^{5} \mathrm{mV} / \mathrm{M}$ for $\mathrm{PC}$-chol bilayers. Thus the present results indicate the basic agreement between 2,4-D induced changes of electric potential difference across GMO monolayers and electric potential differences derived from the changes of kinetics of ion translocation in GMO membranes, $(\Delta \phi$ is the change of the electric potential difference between the membrane interior and the reaction plane that is associated with the change of nonactin- $K^{+}$translocation, whereas $\Delta \psi$ is that between the membrane interior and the aqueous solution that can be associated with the change of the net potential difference across the lipid monolayer). Since $|\Delta \psi|>|\Delta \phi|$ it is to be expected that $\left|b_{m}\right| \geq\left|b_{b}\right|$. Experimental results for GMO confirm such relationships. In contrast, there is no such correspondence between the PC-chol monolayers and bilayers. Its absence is not understood. It is not clear whether the discrepancy is caused by a lower partition coefficient of 2,4-D between PC-chol monolayer and the aqueous medium as compared with that of the bilayer, or is due to the difference in the location and orientation of 2,4-D molecules with respect to the aqueous surface. 


\section{6f. BIOLOGICAL SIGNIFICANCE}

Results of studies of biological activity of 2,4-D can be compared with the effect of 2,4-D on ion transport in lipid membranes. Such a comparison is presented in Table III. It follows that there is a similarity between the action of 2,4-D in biological systems and its effect on ion transport in lipid membranes. The existence of correlation between biological activity and ion transport modification effect suggests that the latter effect is closely related to biological activity of $2,4-D$.

There is, however, an important question that deserves further study and should be answered before it can be stated that the membrane permeability modification of 2,4-D is the primary biological effect. We have found that the effect of 2,4-D on ion transport is dependent upon the $\mathrm{pH}$ of the aqueous medium and that in the biologically relevant $\mathrm{pH}$ range the ion transport modification effect is rather weak.

There appears to be two alternative propositions that should be explored, before a final conclusion between biological and membrane phenomena can be made: 1. Biological activity of 2,4-D is associated with some metabolic product of 2,4-D, rather than 2,4-D molecule itself. 2. In biological membranes, the dissociation characteristics of 2,4-D can be altered due to the presence of negative charge at the membrane surface.

In the former case, one class of possible membrane-active metabolic products are amino acid conjugates of $2,4-D$, since amino acid conjugates are found to be biologically active $(25,27)$. These compounds are present at higher concentrations in plants in which 2,4-D is more 
toxic (26). This is one of the motivations which led us to investigate the effect of amino acid conjugates of 2,4-D on ion transport in lipid membranes.

TABLE III

CORRELATION OF BIOLOGICAL AND BILAYER MEMBRANE PHENOMENA

$$
\begin{array}{cl}
\text { Biological effects (reported } & \text { Effect of } 2,4-D \text { on ion } \\
\text { in literature) } & \text { transport in lipid membranes } \\
& \text { (present work) }
\end{array}
$$

1. Hormonal activity of $2,4-D$ is more prominent in acid media (109). Also greater inhibition of growth of roots was found in the presence of the neutral form of 2,4-D (109). The biological results were explained by the assumption that the neutral form of 2,4-D is active.

2. 2,4-D was found to inhibit the uptake of negatively charged ions $\mathrm{Cl}^{-}$and $\mathrm{NO}_{3}^{-}$into plant roots $(110)$. The biological effect is rapid. It was argued that the effect is primary and not a response to some metabolic disturbance.
1. Only the neutral form of 2,4-D modifies the ion transport in membranes.
2. 2,4-D was found to have the ability to suppress the translocation of negatively charged $\mathrm{TPhB}^{-}$ions in lipid membranes. 
In the latter case, one can argue that if the dissociation characteristics of 2,4-D adsorbed in membrane are altered, it is then possible that neutral 2,4-D molecules are present in membranes at sufficient concentrations to modify the membrane permeability at biological pH. Fromherz et al. have shown that the $\mathrm{pK}_{\mathrm{a}}$ parameter of acids incorporated into monolayers increased for negatively charged surfaces (111). Due to the presence of negatively charged lipids such as phosphatidyl serine, phosphatidyl glycerol and negatively charged proteins, the biological membranes may have a net negative surface charge. This mechanism would also explain modification of ionic permeability of biological membranes at biological $\mathrm{pH}$.

6g. MODIFICATION OF ION TRANSPORT IN LIPID MEMBRANES BY AMINO ACID CONJUGATES OF 2-4-D, INDOLE ACETIC ACID, $2,4,5-\mathrm{T}, 2,4,6-\mathrm{T}, 2,4-\mathrm{DB}$ AND 2,4-D-ISOBUTYL ESTER

The results of the effect of the three amino acid conjugates of 2,4-D, leucine, isoleucine, and valine, on lipid membranes revealed that these compounds modify the transmembrane ion transport of the cation nonactin- $\mathrm{K}^{+}$complex in a similar way to 2,4-D. As shown in Figs. 19-21, in the $\mathrm{pH}$ range from 1 to 5 the modification of ion transport is more prominent at low $\mathrm{pH}$ where these molecules are in the neutral form. At high $\mathrm{pH}$ when the molecules are in the anionic form, the conductivity enhancement of nonactin- $\mathrm{K}^{+}$complex is smaller. These results suggest that the enhancement of the conductance of positive ions is of the same origin as in the case of $2,4-D$, specifically, due to adsorbed neutral molecules of amino acid conjugates (Sec. 3a). 
Although the neutral form of 2,4-D - amino acid conjugates is more effective in modification of transmembrane ion transport of nonactin- $\mathrm{K}^{+}$ complex than the anionic form, at high $\mathrm{pH}(\mathrm{pH} \mathrm{5-8)}$ and high concentration $(800 \mu M)$ of amino acid conjugate in the aqueous solution, there is a significant enhancement of nonactin-K ${ }^{+}$conductance (Figs. 22-24). In this $\mathrm{pH}$ range molecules are mainly in the anionic form because $\mathrm{pK}=$ 3.2-3.5. There are two alternative explanations: (1) The surface charge of the membrane has become more negative due to the adsorbed anions of amino acid conjugates of 2,4-D; and (2) since in the 2,4-D - amino acid conjugates the ionized carboxylic group is further away from the benzene ring than in the 2,4-D anion, the dipole moment of the anion of the amino acid conjugate may be aligned in the same direction as in the neutral molecule. If the latter is the case, the conductivity enhancement of nonactin- $\mathrm{K}^{+}$may be due to both enhanced adsorbed nonactin- $\mathrm{K}^{+}$ complex at the membrane surface as a consequence of increase of the negative surface charge of the membrane, and due to the change of the dipole potential of the interface. It is not possible to separate the two hypotheses. In either case, the enhancement of nonactin- $\mathrm{K}^{+}$conductance is expected to be more or less constant throughout the high pH range since there are no other ionizable groups other than the carboxylic group. As seen in Figs. 22-24 we have observed that the enhanced conductance disappears above pH 8 for all three types of amino acid conjugates. The disappearance of the effect is difficult to understand. We discuss two possible alternatives. The high $\mathrm{pH}$ enhancement effect is either due to (a) presence of 2,4-DCP as an impurity, or (b) the inhomogeneity of lipids used in membrane preparations. We discuss these cases below: 
(a) The case of 2,4-DCP impurity: This compound has $\mathrm{pK}_{2}=7.8$ (101), and as our data indicates (Fig. 25) the neutral form of 2,4-DCP enhances nonactin $-\mathrm{K}^{+}$conductance, and the conductance disappears above $\mathrm{pH}$ 8. This is in qualitative agreement with high $\mathrm{pH}$ effect seen for the amino acid conjugates. However, there is a quantitative disagreement in the amount of 2,4-DCP that would be necessary to produce the effect of the same magnitude. According to our measurements, $20 \%$ of the 2,4-D - amino acid conjugate sample would have to be 2,4-DCP, which is impossible. An aqueous solution of 2,4-DCP of such concentration has a characteristic smell, but the smell is absent for solutions of amino acid conjugates. More sophisticated methods such as thin layer chromatography did not indicate any presence of 2,4-DCP, or other impurities. Another possibility that was explored was the case of spontaneous formation of 2,4-DCP in the aqueous solution at basic $\mathrm{pH}$. This possibility was also rejected because a four-weeks old solution maintained at basic $\mathrm{pH}$ and a fresh solution of isoleucine 2,4-D conjugate produced the same enhancement of nonactin- $\mathrm{K}^{+}$conductance.

(b) The case of lipid inhomogeneity: This question remains open and should be the subject of further studies. Fromherz et al have shown that the $\mathrm{pK}_{\mathrm{a}}$ parameter of acids incorporated into monolayers increased for negatively charged surfaces (111). Measurements of electrophoretic mobility done in our laboratory on vesicles prepared from lipids used in this work indicated small negative surface potential (3-5 $\mathrm{mV}$ in $0.05 \mathrm{M} \mathrm{KC1}$ ), which can be due tto low content of negatively charged lipids not detected by thin layer chromatography. 
Therefore at the present time, the origin of enhanced membrane conductance by amino acid conjugates of $2,4-\mathrm{D}$ at high $\mathrm{pH}$ is not understood.

We have found that $2,4,5-T$ and $2,4-D B$ produce membrane transport effects similar to that of 2,4-D. As shown in Figs. 29 and 30 , the conductance of the cation nonactin $-\mathrm{K}^{+}$complex is enhanced in the presence of $2,4,5-\mathrm{T}$ and $2,4-\mathrm{DB}$. The modification is more prominent at $\mathrm{pH}$ where $2,4,5-\mathrm{T}$ and $2,4-\mathrm{DB}$ are in neutral form. Therefore, the changes in transmembrane ion transport are associated with the neutral molecules. This effect can be understood by the dipole hypothesis,i.e., the neutral molecules of phenoxy acids, 2,4-DB and 2,4,5-T decrease the electric potential of membrane interior.

The above conclusion follows from the changes of voltage dependence of membrane conductance observed in the presence of $2,4,5-T$. As we have shown earlier (see Sec. 6b), the ratio $G(V) / G(0)$ depends on the parameter A which is approximately equal to $2 \mathrm{k}_{I S O} / \mathrm{k}_{\mathrm{D}}$ at $10 \mathrm{w}$ potassium ion concentration (Eq. 28a). The logarithm of the kinetic parameter A vs concentration of $2,4,5-\mathrm{T}$ was plotted in Fig. 43. As this analysis indicates, the logarithm of $A$ increases linearly with the concentration of $2,4,5-\mathrm{T}$. This result is very similar to that observed for 2,4-D (Fig. 33). Assuming again, that the change of the kinetic parameter $A$ is dominated by the change of the height of membrane potential energy barrier and that this change is of electric origin, one can calculate $\Delta \phi$ per mole of $2,4,5-T$ and $2,4-D$ in aqueous solution. The change of electric potential difference between the membrane core and adsorption/reaction plane is $\Delta \phi=b c$, where c is pesticide concentration. We have observed $b_{2,4-D}=1.2 \times 10^{5} \mathrm{mV} / \mathrm{M}$ as compared 


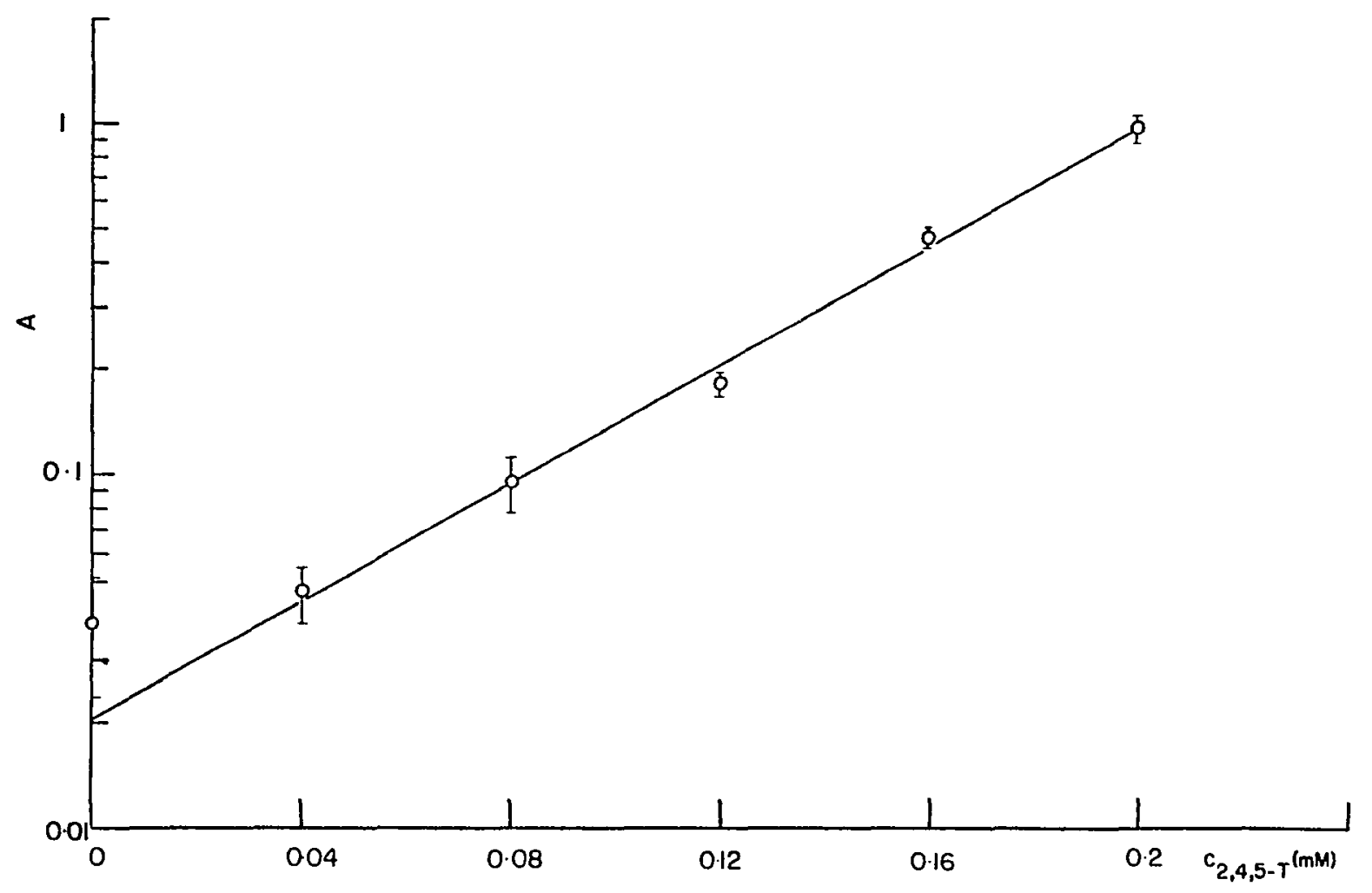

Figure 43. Dependence of the kinetic parameter $A \propto k_{I S O} / k_{D}$ for $P C$-chol membranes on 2,4,5-T concentration. $c_{\text {nonactin }}$ (aqueous sol.) $=1 \times 10^{-7} \mathrm{M}$ $c_{\text {nonactin (membrane sol.) }}=3 \times 10^{-5} \mathrm{M}, \mathrm{c}_{\mathrm{K}^{+}}=0.06 \mathrm{M}$, ionic strength (LiCl + $\mathrm{KC} 1)=1.0 \mathrm{M}, \mathrm{pH}=2$. The solid line represents the linear least square fit of $\log A_{\text {nonactin- }}+\mathrm{Vs}^{\mathrm{c}} \mathrm{C}_{2,4, \mathrm{D}^{*}}$ 
with $b_{2,4,5-\mathrm{T}}=4.8 \times 10^{5} \mathrm{mV} / \mathrm{M}$. Thus $2,4,5-\mathrm{T}$ induced change potential difference between the membrane interior and the adsorption/ reaction plane is about 4 times larger than that of 2,4-D. The parameter $b$ contains a factor which includes the partition coefficient of the compound between membrane and aqueous solution and the normal component of pesticide dipole moment. The dipole moment of 2,4,5-T is unknown, but it is expected to be comparable or larger than that of $2,4-D$

Even though $2,4,5-\mathrm{T}$ and $2,4,6-\mathrm{T}$ are very similar compounds, their effect on Iipid membranes was found to be different. Presence of $0.2 \mathrm{mM}$ of $2,4,5-\mathrm{T}$ enhanced the nonactin- $\mathrm{K}^{+}$conductance across lipid membrane $4 \times 10^{4}$ times while 2,4,6-T enhanced it only 10 times (Fig. 31). The effect of $2,4-D B$ and $2,4-D$ is also much stronger compared to the effect of $2,4,6-T$. The smaller activity of $2,4,6-\mathrm{T}$ on bilayer membranes could be explained by the symmetry of the molecule, due specifically to the symmetry of the ring. Because of this symmetry, the net dipole moment due to chlorine atoms on the benzene ring of $2,4,6-\mathrm{T}$ molecule becomes very small compared to that of asymmetric molecules 2,4,5-T or 2,4-D. This appears to be a general principle. Anderson et al. also found that neutral para and meta nitrophenols change the membrane transport properties of other ions more effectively than ortho nitrophenol which has a smaller dipole moment (66).

The fact that 2,4-D - isobutyl ester does not alter the membrane transport properties while 2,4-D does implies that the ester molecule, when adsorbed on the membrane, is not oriented in such a way that it contributes to the net dipole potential across the membrane. This molecule does not have a polar region and, therefore, it may not be 
able to be oriented at the membrane/water interface the same way as the 2,4-D or amino acid conjugates of 2,4-D.

From the study with $2,4-\mathrm{D}, 2,4-\mathrm{DB}, 2,4,5-\mathrm{T} 2,4,6-\mathrm{T}, 2,4-\mathrm{DCP}$, 2,4-D - amino acid conjugates it follows that the prerequisite for membrane activity is the existence of polar and nonpolar regions of the molecules and asymmetric electron density distribution in the part of the molecule that is inserted into the membrane.

Membrane conductivity measurements of lipid membranes in the presence of the naturally occurring auxin IAA have shown that IAA enhances the transport of cation nonactin $-K^{+}$complex across the membrane (Fig. 27). The effect of IAA on bilayer membranes can be understood in terms of the dipole hypothesis, since this effect is more pronounced when IAA is in the neutral form and the effect disappears at $\mathrm{pH}$ higher than $\mathrm{pK}(\mathrm{pK}=4.75$ (102)).

The apparently common mechanism of action of phenoxy pesticides, some of their biologically active metabolic products, and that of natural auxin IAA on transport in lipid membrane is of interest: (1) There appears to be a correlation between the biological activity and the effect of enhancement of cation transport in membranes. The existence of the enhancement effect for natural auxin IAA gives support to the hypothesis that the biological activity of IAA and presumably of other pesticides based on phenoxy acids, namely, the elongation of cells, is initiated by secretion of hydrogen ions (20, 21). The secretion can result from the enhanced transmembrane transport and positively charged ions. We have found that the dipolar type of mechanism of membrane modification is a very general one, and is 
applicable to any type of transmembrane transport as long as the membrane traversing species is positively charged. 


\section{CHAPTER VII}

CONCLUSIONS, HYPOTHESIS AND PROPOSAL FOR FURTHER STUDIES

In this work the effect of 2,4-D on direct and carrier-mediated transport of ions in lipid membranes has been studied in detail and a model for the action of 2,4-D on ion transport has been proposed. The results on 2,4-D have been used to progress in understanding of the effects of biologically active metabolic products of 2,4-D, amino acid conjugates, indole acetic acid and other phenoxy pesticides and some structural analogs.

7a. CONCLUSIONS DRAWN FROM THE INVESTIGATION OF THE MECHANISM OF MEMBRANE PERMEATOXICITY OF 2,4-D

(a.1) The ionized form of 2,4-D does not permeate through lipid membranes.

(a.2) 2,4-D increases the rate of transport of positively charged lipid soluble ions, and inhibits the transport of negatively charged tetraphenylborate ions.

(a.3) Only the neutral molecules of 2,4-D affects the transport of ions across lipid membranes.

(a.4) 2,4-D changes the kinetics of nonactin-mediated potassium ion transport and the kinetic effects can be understood in terms of simple carrier transport model. It has been shown that one of the major effects of 2,4-D is associated 
with the increase of the rate constant of translocation of the loaded carrier as compared to its dissociaton rate constant. At high 2,4-D concentration the rate of electric field driven potassium ion transport across the membrane is so high that it becomes limited at low potassium concentration by the rate of recombination of nonactin with $\mathrm{K}^{+}$ions and at high potassium concentration by the rate of back diffusion of unloaded nonactin molecules.

(a.5) Suppression of transport of negative $\mathrm{TPhB}^{-}$ions in $\mathrm{PC}-$ cholesterol membranes by $2,4-\mathrm{D}$ is dominated by the decrease of the probability of ion translocation across the membrane as indicated by the increase of the current relaxation time constant. The effect of 2,4-D on $\mathrm{TPhB}^{-}$adsorption at the membrane/water interface is rather small.

(a.6) The rate of transport of tetraphenylarsonium ${ }^{+}$ions across the membrane as well as the ratio of $k_{I S O} / k_{D}$ of nonactin- $K^{+}$ exponentially increase with the concentration of 2,4-D and ionic strength. From these data and the results of $\mathrm{TPhB}^{-}$current relaxation studies, it has been possible to determine the changes of the electric potential of the membrane interior, which are negative and as large as 140$180 \mathrm{mV}$.

(a.7) The results of studies of $\mathrm{TPhB}^{-}$current relaxation indicate that about $25-30 \%$ of the total induced change of the potential difference is between the plane of the adsorption of 
$\mathrm{TPhB}^{-}$and the aqueous solution. The remaining fraction is between the membrane interior and the adsorption plane.

(a.8) Within the framework of the barrier model of lipophilic ion transport, the 2,4-D - induced changes of electric potential difference between the central plane of the membrane and the adsorption-reaction plane for $\mathrm{TPhB}^{-}$and nonactin- $\mathrm{K}^{+}$ions have been found to be very similar. The changes of kinetics of transport of positive and negative ions also suggest that, except for the change of the electric potential, the ion transport properties in the membrane interior are not affected by the presence of 2,4-D. The effect of 2,4-D on ion transport across the membrane interior can be reduced to the change of electric potential at the edge of membrane hydrocarbon region. The 2,4-D related electric potential difference is proportional to 2,4-D concentration in the aqueous solution.

(a.9) The results of measurements of interfacial potential difference across air/water and air/lipid monolayer/water boundaries as a function of 2,4-D concentration, $\mathrm{pH}$ and ionic strength indicate that the electric potential of the nonpolar side of the interface becomes more negative in the presence of neutral 2,4-D molecules, and that the magnitude of this effect increases with the increasing ionic strength of the electrolyte. Since these features are displayed by interfaces of both types, as well as by lipid bilayer membranes, the results of interfacial potential measurements suggest that the action of 2,4-D in lipid membranes is not 
associated with the change of orientation of lipid

molecule adsorbed at the nonpolar/polar boundary region of the membrane.

(a.10) The action of 2,4-D on ion transport in lipid membranes is consistent with the hypothesis that the dipole moment of 2,4-D molecules adsorbed within the membrane interfacial region, is directed toward the aqueous medium. The results of kinetic studies of nonactin-mediated transport of potassium and tetraphenylborate relaxation studies suggest that the layer of 2,4-D molecules is predominently located below the plane of recombination of nonactin and $\mathrm{K}^{+}$ions and tetraphenylborate adsorption plane, that is on the hydrocarbon side of the interface.

(a.11) The results suggest that there is a correlation between the biological activity of 2,4-D and its ability to modify the rate of transport of positive and negative ions in lipid membranes.

7b. CONCLUSIONS AND HYPOTHESES DRAWN FROM THE RESULTS OF THE STUDIES OF AMINO ACID CONJUGATES OF 2,4-D, INDOLE ACETIC ACID, 2,4-DB, 2,4,5,-T, 2,4,6-T AND 2,4-D ESTER

(b.1) The ionized form of these compounds does not permeate through bilayer lipid membranes.

(b.2) Neutral molecules of leucine, isoleucine and valine amino acid conjugates of $2,4-D$ were found to be transport active; the effect is similar to that of 2,4-D. The modification of the transport of nonactin-mediated $\mathrm{K}^{+}$ions at high $\mathrm{pH}$ 
range $(5-10)$ by amino acid conjugates may be due to the presence of negatively charged lipids in the membranes which have been used. This possibility has to be experimentally verified with negatively charged lipid membranes.

(b.3) The action of natural plant growth hormone, indole acetic acid, is similar to that of phenoxy pesticides.

(b.4) The available data indicate that the initial biological effect of phenoxy pesticides is identical to that of the above natural auxin, whose effect is associated with translocation of hydrogen ions. We propose that the nature of biological action of pesticides of phenoxy group is the enhancement of membrane permeability of positively charged ions, and suppression of membrane permeability of negative ions. This hypothesis should be experimentally verified on a membrane transport system of biological origin.

(b.5) We propose that two conditions have to be satisfied for the existence of membrane transport modification activity by dipolar mechanism: (1) The existence of distinctively polar and nonpolar regions in the membrane modifying molecules, which is essential for proper orientation of molecules adsorbed at the membrane surface; (2) The existence of a net dipole moment associated with the asymmetric distribution of electrons in the nonpolar part of membrane modifying molecules. This hypothesis should be tested experimentally on a series of structural analogs of the various substances discussed in this section. The dipole moments of possible 
analogs would have to be determined experimentally and/or theoretically, and the partition coefficients between water and lipid would also have to be measured. 


\section{REFERENCES}

1. Singer, S. J. and G. L. Nicholson, 1972, The fluid mosaic model of the structure of cell membranes. Science. 175:720.

2. Eisenberg, M. and S. McLaughlin, 1976, Lipid bilayers as models of biological membranes. Bio Science. 26:436.

3. Engleman, D. M., 1971, Lipid bilayer structure in the membrane of mycoplasma laidlawii. J. Mol. Biol. 58:153.

4. Stein, J. M., Tourtellolte, M. E., J. C. Reinert, R. N. McElhaney and R. L. Radar, 1969, Calorimetric evidence for the liquidcrystalline state of lipids in a biomembrane. Proc. Nat. Acad. Sci. USA. 63:104

5. Reinert, J. C. and J. M. Stein, 1970, Calorimetric detection of a membrane lipid phase transition in living cells. Science. $168: 1580$.

6. Branton, D. and D. W. Deamer, 1972, In Membrane structure. Springer Verlag, New York. 70.

7. McLaughlin, A. C., P. R. Cullis, M. A. Herminga, D. I. Hoult, G. K. Radda, G. A. Ritchie, P. J. Seeley and R. E. Richards. 1975. Applicaton of 3lP NMR to model and biological membrane systems. FEBS letters. 57:213.

8. White, S. H. and T. E. Thompson, 1973, Capacitance, area and thickness variations in thin lipid films. Biochim. Biophys. Acta. 323:7.

9. TiTien, H., 1974, In Bilayer lipid membranes (BLM), Theory and Practical. Marcel Dekker Inc., New York. 1.

10. Muller, P. and D. O. Rudin, 1969, Translocators in bimolecular lipid membranes: their role in dissipative and conservative bioenergy transductions. Curr. Top. Bio. Energ. 3:157.

11. Hille, B., 1970, Ionic channels in nerve membranes. Prog. Biophys. Mol. Biol. $21: 1$.

12. Mitche11, P., 1966, Chemiosmotic coupling in oxidative and photosynthetic phosphorylation. Bio. Rev. 41:445.

13. Lotlikar, P. D., L. F. Remmert and V. H. Freed, 1968, Effects of 2,4-D and other herbicides on oxidative phosphorylation in mitochondria from Cabbage. Weed Sci. 16:161. 
14. Overbeek, J. V., 1964, Survey of mechanisms of herbicide action. In Physiology and Biochemistry of Herbicides, L. J. Hudus, editor. Academic Press, New York. 387.

15. Murray, M.G. and J. L. Key, 1978, 2-4-dichlorophenoxyacetic acidehanced phosphorylation of soybean nuclear proteins. Plant Physiol. $61: 190$.

16. Rip, J. W. and J. H. Cherry, 1976, Liver enlargement induced by the herbicide 2,4,5-trichlorophenoxyacetic acid $(2,4,5-\mathrm{T})$. J. Agric. Food Chem. $24: 245$.

17. Berwick, P., 1970, 2,4-dichlorophenoxyacetic acid poisoning in man. J. Amer. Med. Assoc. 214:1114.

18. Hofmann, W. W., W. Alston, and G. Rowe, 1966, A study of individual neuromuscular junctions in myotonia. Electroencephalogr. Clin. Neurophysiol. 21:521.

19. Dux, E., I. Toth, L. Dux, F. Joo and G. Kysely, 1977, The possible cellular mechanism of 2,4-dichlorophenoxyacetic-induced myopathy. Febs. Lett. $82: 219$.

20. Cleland, R., 1973, Auxin induced hydrogen ion excretion from Avena Coleoptiles. Proc. Nat. Acad. Sci. USA, 70:3092.

21. Mentze, J., B. Raybound, J. D. Cohen and D. L. Rayle, 1977, Auxininduced $\mathrm{H}^{+}$secretion in Helianthus and its implications. Plant. Physiol. 60:509.

22. Feung, C. S., R. H. Hamilton and F. H. Witham, 1971, Metabolism of 2,4-dichlorophenoxy acetic acid by soybean cotyledon callus tissue cultures. J.Agric. Food Chem. 19:475.

23. Feung, C. S., R. H. Hamilton, F. H. Witham and R. 0. Mumma, 1972, The relative amounts and identification of some 2,4dichlorophenoxyacetic acid metabolities isolated from soybean cotyledon callus cultures. Plant Physiol. 50:80.

24. Feung, C. S., R. H. Hamilton and R. O. Mumma, 1973, Metabolism of 2,4-dichlorophenoxyacetic acid. V. Identification of metabolites in soybean callus tissue cultures. J.Agric. Food Chem. 21:637.

25. Feung, C. S., R. O. Mumma and R. H. Hamilton, 1974, Metabolism of 2,4-dichlorophenoxyacetic acid. VI. Biological properties of amino acid conjugates. J. Agric. Food. Chem. 22:307.

26. Feung, C. S., R. H. Hamilton and R. 0. Mumma, 1975, Metabolism of 2,4-dichlorophenoxyacetic acid. VII. Comparison of metabolism of five species of plant callus tissue cultures. J. Agric. Food Chem. 23:373. 
27. Feung, C. S., R. H. Hamilton and R. O. Mumma, 1977, Metabolism of 2,4-dischlorphenoxyacetic acid. II. Herbicidal properties of amino acid conjugates. J. Agric. Food Chem. 25:878.

28. Bristol, D. W., A.M. Ghanuni and A. E. Oleson, 1977, Metabolism of 2,4-dichlorophenoxyacetic acid by wheat cell suspension cultures. J.Agric. Food Chem. 25:1308.

29. Leblanc, O.H., 1969, Tetraphenylborate conductance through lipid bilayer membranes. Biochim. Biophys. Acta. 193:350.

30. Liberman, E. A., and V. P. Topaly, 1969, Permeability of bimolecular phospholipid membranes for lipid soluble ions. Biofizika. $14: 452$.

31. Ketterer, B., B. Neumcke, and P. Lauger, 1971, Transport mechanism of hydrophobic ions through lipid bilayer membranes. J. Membrane. Biol. 5:225.

32. De Levie, R., N. G. Seidah, and D. Larkin, 1974, Tetraphenylborate adsorption onto an artificial ultrathin membrane. J. Electroanal. Chem. $49: 153$.

33. Bruner, L. J., 1975, The interaction of hydrophobic ions with lipid bilayer membranes. J. Membrane Biol. 22:125.

34. Gavach, C., and R. Sandeaux, 1975, Non-mediated zero voltage conductance of hydrophobic ions through bilayer lipid membranes. Biochim. Biophys. Acta $413: 33$.

35. Andersen, O.S., and M. Fuchs, 1975, Potential energy barriers to ion transport within lipid bilayers. Biophys. J. 15:795.

36. De Levie, R., and D. Vukadin, 1975, Dipicrylamine transport across ultrathin phosphatidylethanolamine membranes. J. Electroanal. Chem. 62:95.

37. Benz, R., P. Lauger, and K. Janko, 1976, Transport kinetics of hydrophobic ions in lipid bilayer membranes. Charge pulse relaxation studies. Biochim. Biophys. Acta. 455:701.

38. Wulf, J., R. Benz, and W. G. Poh1, 1977, Properties of bilayer membranes in the presence of dipicrylamine. A comparative study by optical adsorption and electrical relaxation measurements. Biochim. Biophys. Acta. 465:429.

39. Benz, R., and P. Lauger, 1977, Transport kinetics of depicrylamine through lipid bilayer membranes: effects of membrane structure, Biochim. Biophys. Acta. 468:245.

40. Andersen, O.S., S. Feldberg, H. Nakadomari, S. Levy, and S. McLaughlin, 1978, Electrostatic interactions among hydrophobic ions in lipid bilayer membranes. Biophys. J. $21: 35$ 
41. Feldberg, S. W., and A. B. Deldago, 1978, Inner voltage clamping. A method for studying interactions among hydrophobic ions in a lipid bilayer. Biophys. J. 21:71.

42. Wang, C. C., and L. J. Bruner, 1978, Dielectric saturation of the aqueous boundary layers adjacent to charged bilayer membranes. J. Membrane Biol. 38:1.

43. Lea, E. J. A., and C. P. Croghan, 1969, The effect of 2,4dinitrophenol on the properties of thin lipid films. $\mathrm{J}$. Membrane Biol. 1:225.

44. Finkelstein, A., 1970, Weak-acid uncouplers of oxidative phosphorylation. Mechanism of action on thin lipid membranes. Biochim. Biophys. Acta. $205: 1$.

45. Hopfer, U., A. L. Lehninger, and W. J. Lenarz, 1970, The effect of polar moiety of lipids on bilayer conductance induced by uncouplers of oxidative phosphorylation. J. Membrane Biol. $3: 142$.

46. Leblanc, 0. H., 1971, The effect of uncouplers of oxidative phosphorylation on lipid bilayer membranes: carbonylcyanide m-chlorophenylhydrazone. J. Membrane Biol. 4:227.

47. Foster, M. and S. McLaughlin, 1974, Complexes between uncouplers of oxidative phosphorylation. J. Membrane Bio1. 17:155.

48. Neumcke, B., and E. Bamberg, 1975, The action of uncouplers on lipid bilayer membranes. In Membranes, Vol. 3, G. Eisenman, editor. Marce1 Dekker, New York. 215.

49. Gre11, E., T. Funck, and F. Eggers, 1975, Structure and dynamic properties of ion-sepcific antibiotics. In Membranes, Vol. 3, G. Eisenman, editor. Marcel Dekker, New York. 1.

50. Ferguson, S.M.F., Estrada-o, and H. Lardy, 1971, Potassium-specific uncoupling by nigericin. J. Biol. Chem. 246:5645.

51. Toro, M., C. Gomez-Lojero, M. Montal, and S. Estrada-o, 1976, Charge transfer mediated by nigericin in black lipid membranes. Bioenergetics. 8:19.

52. Markin, V. S., V. S. Sokolov, L. I. Boguslavsky, and L. S. Jaguzhinsky, 1975, Nigericin-induced charge transfer across membranes. J. Membrane Biol. 25:23.

53. McLaughlin, S., A. Bruder, S. Chen, and C. Moser, 1975, Chaotropic anions and the surface potential of bilayer membranes. Biochim. Biophys. Acta. 394:304.

54. McLaughlin, S., 1972, The mechanism of action of DNP on phospho1ipid bilayer membranes. J. Membrane Biol. 9:361. 
55. White, S. H., 1973, The surface charge and double layers of thin lipid films formed from neutral lipids. Biochim. Biophys. Acta. $323: 343$.

56. Hsu, K., R. Jayaweera and P. Smejtek, 1978. Unpublished results.

57. Haydon, D. A., and V. B. Myers, 1973, Surface charge, surface dipoles and membrane conductance. Biochim. Biophys. Acta. $307: 429$.

58. Hladky, S. B., and D. A. Haydon, 1973, Membrane conductance and surface potential. Biochem. Biophys. Acta. 318:464.

59. Haydon, D. A., 1975, Functions of the lipid in bilayer ion permeability. Ann. N.Y. Acad. Sci. 264:2.

60. Szabo, G., 1974, Dual mechanism for the action of cholesterol on membrane permeability. Nature (London). 252:47.

61. Szabo, G., 1976, The influence of dipole potentials on the magnitude and kinetics of ion transport in lipid bilayer membranes. In Extreme Environments. Heinrich, M.R., editor, Academic Press, New York. 321.

62. Szabo, G., 1977, Mechanisms by which small molecules alter ionic permeability through lipid bilayer membranes. In Membrane Toxicity. Miller, M. W., and A. E. Shamoo, editors. Plenum press. 167.

63. Benz, R., 0. Frohlich, and P. Lauger, 1977, Influence of membrane structure on the kinetics of carrier-mediated ion transport through lipid films. Biochim. Biophys. Acta. 464:465.

64. Benz, R., and D. Cros, 1978, Influence of sterols on ion transport through lipid bilayer membranes. Biochim. Biophys. Acta. $506: 265$.

65. Pickar, A. D., and R. Benz, 1978, Transport of oppositely charged lipophilic probe ions in lipid bilayer membranes having various stmuctures. J. Membrane Biol. 44:353.

66. Andersen, 0.S., A. Finkelstein, I. Katz, and A. Cass, 1976, Effect of phloretin on the permeability of thin lipid membranes. J. Gen. Physiol. 67:749.

67. Melnik, E., R. Latorre, J. E. Hall, and D. C. Tosteson, 1977 , Phloretin-induced changes in ion transport across lipid bilayer membranes. J. Gen. Physiol. 69:243.

68. Wang, C. C., and L. J. Bruner, 1978, Lipid-dependent and phloretininduced modifications. of dipicrylamine adsorption by bilayer membranes. Nature (London). 272:268. 
69. De Levie, R., S. K. Rangarajan, P.F. Seelig, and 0.S. Andersen, 1979, On the adsorption of Phloretin onto a black lipid membrane. Biophys. J. $25: 295$.

70. McLaughlin, S., 1973, Salicylates and phospholipid bilayer membranes. Nature (London) . 243:234.

71. Borisova, M., L. N. Ermishkin, E. A. Liberman, A. Y. Silberstein, and E. M. Trofimov, 1974, Mechanisms of conductivity of bimolecular lipid membranes in the presence of tetrachlorotrifluoremethylbenzimidazole, J. Membrane Biol. 18:243.

72. Cohen, F. S., M. Eisenberg, and S. McLaughlin, 1978, The kinetic mechanism of action of an uncoupler of oxidative phosphorylation. J. Membrane Biol. 37:361.

73. Kuo, K. H., T. R. Fukuto, T. A. Miller, and L. J. Bruner, 1976, Blocking of valinomycin-mediated bilayer membrane conductance by substituted benzimidazoles. Biophys. J. 16:143.

74. Kuo, K. H., and L. J. Bruner, 1976, Modification of valinomycinmediated bilayer membrane conductance by $4,5,6,7$-tetrachloro2-methylbenzimidazole. J. Membrane Biol. 26: 385 .

75. Neumcke, B., and P. Lauger, 1969, Nonlinear electrical effects in lipid bilayer membranes. II. Integration of the generalized Nernst-Planck equation. Biphys. J. 9:1160.

76. Lauger, P. and B. Neumcke, 1973, Theoretical analysis of ion conductance in lipid bilayer membranes. In Membranes. G. Eisenman, editor. Marcel Dekker Inc., New York. 2:1.

77. MacDonald, R. C., and A. D. Bagham, 1972, Comparison of double layer potentials in lipid monolayers and lipid bilayer membranes. J. Membrane Biol. 7:29.

78. McLaughlin, S., 1977, Electrostatic potentials at membrane solution interfaces. In Current Topics in Membranes and Transport. F. Bronnex and A. Kleinzeller, editors. Academic Press, New York. 9:71.

79. McLaughlin, S., and M. Eisenberg, 1975, Antibiotics and membrane biology. Annu. Rev. Biophys. Bioenerg. 4:335.

80. McLaughlin, S., G. Szabo, G. Eisenman, and S. M. Ciani, 1970, Surface charge and conductance of phospholipid membranes. Proc. Nat. Acad. Sci. 67:1288.

81. Hladky, S. B., 1974, The energy barriers to ion transport by nonactin across thin lipid membranes. Biochim. Biophys. Acta. $352: 71$. 
82. Bangham, A. D. and D. Papahadjopoulos, 1966, Biophysical properties of phospholipids. 1. Interaction of phosphatidylserine monolayers with metal ions. Biochim. Biophys. Acta. 126:181.

83. Papahadjopoulos, D., 1968, Surface properties of acidic phospholipids: Interactions of monolayers and hydrated liquid crystals with uni- and bi-valent metal ions. Biochim. Biophys. Acta. $163: 240$.

84. Paltauf, F., H. Hauser and M. C. Phillips, 1971, Monolayer characteristics of some 1,2-Diacyl, 1-alkyl-2acyl and 1,2-dialkyl phospholipids at the air-water interface. Biochim. Biophys. Acta. 249:539.

85. LeBlanck, O. H., 1970, Membrane models? Ion transport through interfaces and lipid bilayers. Biophys. Soc. Abst. TPM-A3: 94 a.

86. Parkin, B., and H. Eyring, 1954, In Ion transport across membranes. H.T. Clarke, editor. Academic Press, New York.

87. B. J. Zwolinski, H. Eyring and C. E. Reese, 1949, Diffusion and membrane permeability I. J. Phys. Colloid. Chem. 53:1426.

88. Laprade, R., S. Ciani, G. Eisenman and G. Szabo, 1975, The kinetics of carrier-mediated ion permeation in lipid bilayers and its theoretical interpretation. In Membranes. G. Eisenman, editor. Marcel Dekker, New York, 3:127.

89. Krasne, S., and G. Eisenman, 1976, Influence of molecular variation of ionophore and lipid on the selective ion permeability of membranes. I. Tetranactin and the methylation of nonactintype carriers. J. Membrane Biol. 30:1.

90. Hladky, S. B., 1975, Tests of the carrier model for ion transport by nonactin and trinactin, Biochim. Biophys. Acta. 375:327.

91. Hladky, S. B., 1975, Steady-state ion transport by nonactin and trinactin. Biochim. Biophys. Acta. 375:350.

92. Hladky, S. B., 1972, The steady-state theory of the carrier transport of ions. J. Membrane Biol. 10:67.

93. Wang, C. C. and L. J. Bruner, 1978, Evidence for a discrete charge effect within lipid bilayer membranes, Biophys. J. 24: 749.

94. Muller, P., D. 0. Rudin, H. T. Teen and W. C. Wescott, 1964, Formation and properties of bimolecular lipid membranes. In Recent Progress in Surface Science. J. E. Danie11, K.G.A. Pankhurst, and A. C. Reddifer, editors. Academic Press, Inc. New York, 1:379. 
95. Hanai, T., D. A. Haydon and J. Taylor, 1965, The variation of capacitance and conductance of bimolecular lipid membranes with area. J. Theoret. Biol. 9:433.

96. Sargent, D. I., 1976, An apparatus for the measurement of very small membrane relaxation currents. Annal. Biochem. 70:100.

97. Smejtek, P., K. Hsu and W. H. Perman, 1976, Electrical conductivity of lipid bilayer membranes induced by pentachlorophenol. Biophys. J. 16:319.

98. Wood, J. W. and D. Fontaine, 1952, Synthetic plant-growth regulators. III. 2,4-dichlorophenoxyacetyl derivatives of amino acids. J. Org. Chem. 17:891.

99. Nelson, N. H., and S. D. Faust, 1969, Acid dissociation constants of selected aquatic herbicides. Env. Sci. Technology. 3:1186.

100. Baily, G. W., and J. L. White, 1965, Herbicides: a compilation of their physical, chemical and biological properties. In Residue Reviews. Springer-Verlag. New York. 10:97.

101. Blackman, G. E., M. H. Parke, and G. Garton, 1955, The physiological activity of substituted phenols. 1. Relationship between chemical structure and physiological activity. Archives of Biochim. Biophys. $54: 45$.

102. The Merck Index, 8th edition, 1968, P. G. Stecher, editor, 565.

103. Thimann, K. V., 1969, The Auxins. In The physiology of plant growth and development. Malcolm B. Wilkins, editor. McGraw Hill, London. 3.

104. R. L. Wain and C. H. Fawcett, 1969, Chemical plant growth regulation. In Plant Physiology a treatise. F. C. Steward, editor. Academic Press, New York, 5a:231.

105. van Overbeek, J., R. Blondeau and V. Horne, 1951, Differences in activity between 2,4-D and other auxins, and its significance in herbicidal action. Plant Physiol. 26:687.

106. Mate11, M., and S. Lindenfors, 1957, Thermodynamic dissociation constants of 2,4-D and some related plant growth regulators. Acta. Chem. Scand. $11: 324$.

107. Bockris, J. O'M., and A.K.N. Reddy, 1971, In Modern Electrochemistry. Plenum Press, New York. $1: \overline{210}, 2: 730$.

108. McClellan, A. L., 1974, In Tables of experimental dipole moments. Rahara Enterprises, E1 Cerrito, CA. 2:281. 
109. Andus, L. J., 1948, Studies on the pH-relationships of root growth and its inhibition by 2,4-dichlorophenoxy acetic acid and coumarin. New Phytol. 48:97.

110. Nance, J.F., 1949, Inhibition of salt accumulation of excited

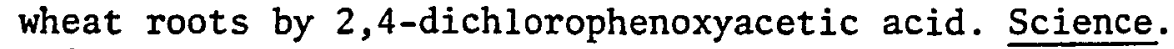
$109: 174$.

111. Fromherz, P. and B. Masters, 1974, Interfacial pH at electrically charged lipid monolayers investigated by the lipid pHindicator method. Biochim. Biophys. Acta. 356:270. 